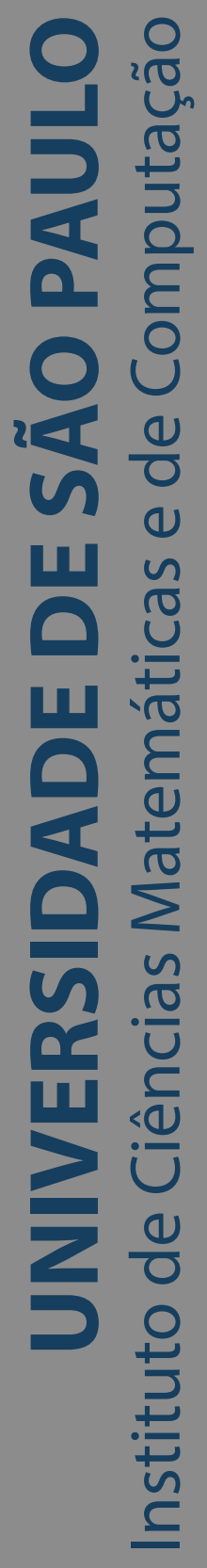

\title{
Equações diferenciais ordinárias e suas aplicações
}

\section{Luiz Henrique da Silva}

Dissertação de Mestrado do Programa de Mestrado Profissional em Matemática em Rede Nacional (PROFMAT) 

Assinatura:

\title{
Luiz Henrique da Silva
}

\section{Equações diferenciais ordinárias e suas aplicações}

\begin{abstract}
Dissertação apresentada ao Instituto de Ciências Matemáticas e de Computação - ICMC-USP, como parte dos requisitos para obtenção do título de Mestre em Ciências - Mestrado Profissional em Matemática em Rede Nacional. VERSÃO REVISADA Área de Concentração: Mestrado Profissional em Matemática em Rede Nacional

Orientadora: Profa. Dra. Michelle Fernanda Pierri Hernandez
\end{abstract}


Ficha catalográfica elaborada pela Biblioteca Prof. Achille Bassi e Seção Técnica de Informática, ICMC/USP, com os dados inseridos pelo(a) autor(a)

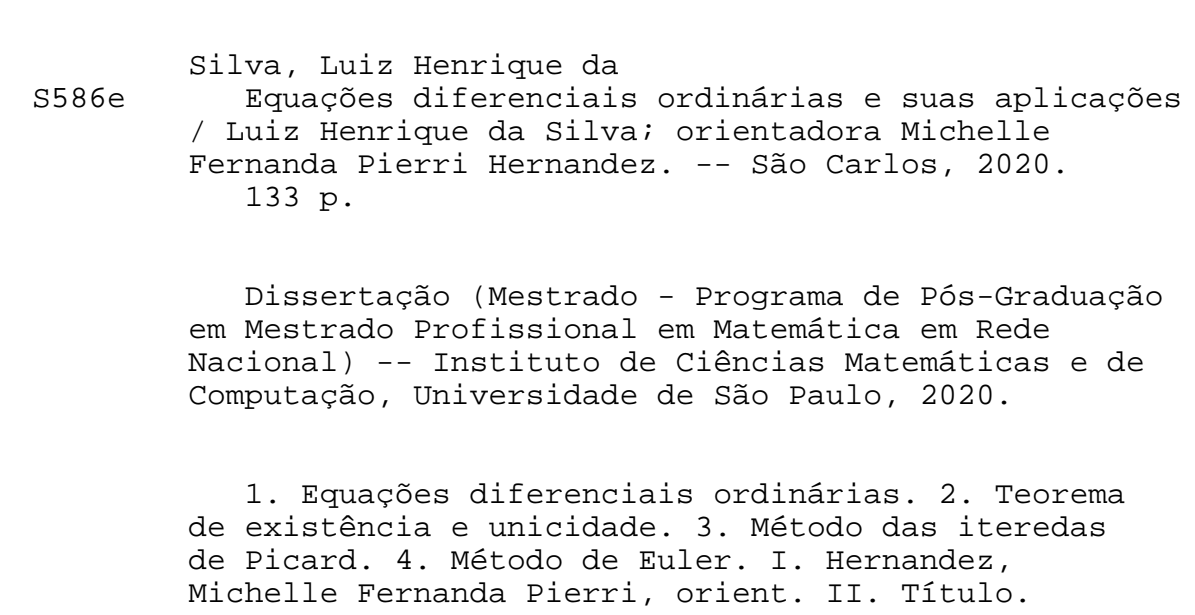

Bibliotecários responsáveis pela estrutura de catalogação da publicação de acordo com a AACR2 


\title{
Luiz Henrique da Silva
}

\section{Ordinary differential equations and their applications}

\author{
Master dissertation submitted to the Institute of \\ Mathematics and Computer Sciences - ICMC-USP, in \\ partial fulfillment of the requirements for the degree of \\ Mathematics Professional Master's Program. FINAL \\ VERSION \\ Concentration Area: Professional Master Degree \\ Program in Mathematics in National Network
}

Advisor: Profa. Dra. Michelle Fernanda Pierri Hernandez 

A Deus por permitir que tudo acontecesse.

Aos meus pais, Leiliane e Luiz Antonio, e ao meu irmão, Jean, pelo apoio e incentivo incondicionais.

À minha orientadora, Michelle, pela dedicação e conhecimento compartilhado.

À minha madrinha, Délvia, pelo apoio.

Aos meus professores pelos ensinamentos.

Aos meus professores de graduação, Daniele, Diogo, Fernando e Karina, pelo incentivo.

Aos meus colegas de turma pela amizade e companheirismo.

Aos meus amigos que direta ou indiretamente contribuíram para esta etapa.

Muito obrigado! 

"Sarah Jane: $O$ universo tem que seguir em frente. A dor e a perda nos definem assim como a felicidade e o amor. Seja um mundo ou um relacionamento tudo tem seu tempo e tudo acaba."

(Toby Whithouse, Doctor Who - Reunião escolar, 2006) 



\section{RESUMO}

SILVA, L. H. Equações diferenciais ordinárias e suas aplicações. 2020. 133 p. Dissertação (Mestrado em Ciências - Mestrado Profissional em Matemática em Rede Nacional) Instituto de Ciências Matemáticas e de Computação, Universidade de São Paulo, São Carlos SP, 2020.

Neste trabalho, estudamos alguns conceitos da teoria de equações diferenciais ordinárias bem como as demonstrações de teoremas de existência e unicidade para equações diferenciais ordinárias de primeira e segunda ordens. Também apresentamos alguns métodos de resolução para essas equações e várias aplicações relacionadas a esses métodos. Finalizamos o trabalho estudando os métodos de Euler e Euler aperfeiçoado, ambos métodos numéricos, para equações diferenciais ordinárias de primeira ordem e descrevemos uma aplicação feita com alunos do ensino médio utilizando esses dois métodos.

Palavras-chave: Equações diferenciais ordinárias, Teorema de existência e unicidade, Método das iteradas de Picard, Método de Euler. 



\section{ABSTRACT}

SILVA, L. H. Ordinary differential equations and their applications. 2020. 133 p. Dissertação (Mestrado em Ciências - Mestrado Profissional em Matemática em Rede Nacional) Instituto de Ciências Matemáticas e de Computação, Universidade de São Paulo, São Carlos SP, 2020.

In this work, we study some concepts of the theory of ordinary differential equations as well as the proofs of existence and uniqueness theorems for differential equations first and second order ordinary shares. We also present some methods of solving these equations and several applications related to these methods. We finished the work studying the methods of Euler and improved Euler, both numerical methods, for ordinary differential equations of first order and describe an application made with high school students using these two methods.

Keywords: Ordinary differential equations, Existence and uniqueness theorem, Picard's iterate method, Euler method. 



\section{LISTA DE ILUSTRAÇÕES}

Figura 1 - Retas $r$ e $s$ delimitando a região em que se encontram os termos da sequência de Picard . . . . . . . . . . . . . . . . . . . . . . 33

Figura 2 - Comportamento das soluções de (3.17) . . . . . . . . . . . . 70

Figura 3 - Comportamento das soluções de (3.19) . . . . . . . . . . . . . 73

Figura 4 - Comportamento das soluções de (3.21) . . . . . . . . . . 75

Figura 5 - Catenária . . . . . . . . . . . . . . . 79

Figura 6 - Uma aproximação pela reta tangente . . . . . . . . . . . . . . . 120

Figura 7 - Gráfico da solução e da aproximação pela reta tangente visto no Exemplo 60122

Figura 8 - Aproximação da integral por um retângulo . . . . . . . . . . . . . 124

Figura 9 - Aproximação da integral por um trapezoide . . . . . . . . . . . 125

Figura 10 - Comparação das soluções . . . . . . . . . . . . . . . . . 130 



\section{LISTA DE TABELAS}

Tabela 1 - Análise do erro do método de Euler . . . . . . . . . . . . . . . . . . 123

Tabela 2 - Análise do erro do método de Euler aperfeiçoado . . . . . . . . . . . . 127 

INTRODUÇÃO . . . . . . . . . . . . . . . . 19

2 EQUAÇÕES DIFERENCIAIS ORDINÁRIAS E UM TEOREMA DE EXISTÊNCIA E UNICIDADE . . . . . . . . . . . . . . . . 21

$2.1 \quad$ Equações Diferenciais Ordinárias . . . . . . . . . . . . . . 21

$2.2 \quad$ Problemas concretos envolvendo EDOs . . . . . . . . . . . . . . . 24

2.2.1 Variação da Temperatura de um Corpo . . . . . . . . . . . . . . . . . 24

2.2.2 Decaimento Radioativo . . . . . . . . . . . . . . . . . . . . . 24

2.2.3 Corpo em Queda Livre com Influência do Atrito . . . . . . . . . . . . 25

2.2.4 Modelos Populacionais . . . . . . . . . . . . . . . . 25

2.2.4.1 Modelo de Malthus . . . . . . . . . . . . . . . . . . . 25

2.2.4.2 Modelo de Verhulst . . . . . . . . . . . . . . . 25

2.2.4.3 Modelo Presa-predador . . . . . . . . . . . . . . . . 26

$2.3 \quad$ Existência e Unicidade de Soluções . . . . . . . . . . . . . . 27

3 EQUAÇÕES DIFERENCIAIS ORDINÁRIAS DE PRIMEIRA ORDEM 41

3.1 Equações Diferenciais Lineares . . . . . . . . . . . . . . 41

3.1.1 Equações Homogêneas . . . . . . . . . . . . . . . . . . 42

3.1.2 Equações Não Homogêneas . . . . . . . . . . . . . . . . . . . . 44

3.1.3 Algumas Aplicações . . . . . . . . . . . . . . . . . 46

3.1.3.1 Modelo Populacional de Malthus . . . . . . . . . . . . . . . . . . . 46

3.1.3.2 Decaimento Radioativo . . . . . . . . . . . . . . . . . 47

3.1.3.3 Diluição de Misturas . . . . . . . . . . . . . . . . . . . . . . . 49

3.1.3.4 Variação da Temperatura de um Corpo . . . . . . . . . . . . . . . . . . 50

3.1.4 A Equação de Bernoulli . . . . . . . . . . . . . . . . . . . . 53

3.1.5 A Equação de Ricatti . . . . . . . . . . . . . . . . . . . . . 55

$3.2 \quad$ Equações Diferenciais Não Lineares . . . . . . . . . . . . 56

3.2.1 Equações Exatas . . . . . . . . . . . . . . . . . 56

3.2.2 Equações com Variáveis Separáveis . . . . . . . . . . . . . 60

3.2.2.1 Equações Autômomas . . . . . . . . . . . . . . . . . . . . . . . 62

3.2.3 Equações Homogêneas . . . . . . . . . . . . . . . . . . . 63

3.2.4 Fatores Integrantes . . . . . . . . . . . . . . . . 65

3.2.5 Algumas Aplicações . . . . . . . . . . . . . . 68 
3.2.5.1 Modelo Populacional de Verhulst . . . . . . . . . . . . . . . . . 68

3.2.5.2 Reação Química . . . . . . . . . . . . . . . . . . . . 76

3.2.5.3 Catenária . . . . . . . . . . . . . . . . . 78

3.2.5.4 Corpo em Queda Livre com Influência do Atrito . . . . . . . . . . . . . . 81

4 EQUAÇÕES DIFERENCIAIS ORDINÁRIAS DE SEGUNDA ORDEM 83

4.1 Equações Diferenciais Lineares de Segunda Ordem . . . . . . . . . 84

4.1.1 Equações Lineares Homogêneas . . . . . . . . . . . . . . . . . 86

4.1.1.1 Método da Redução de Ordem . . . . . . . . . . . . . . . . . . . 88

4.1.1.2 Equações Homogêneas com Coeficientes Constantes . . . . . . . . . . . . 90

4.2 A Equação Não Homogênea . . . . . . . . . . . . . . . . . . 96

4.2.1 Método dos Coeficientes a Determinar . . . . . . . . . . . . . . . . 97

4.2.2 Método da Variação de Parâmetros . . . . . . . . . . . . . . . . 105

4.3 Equação de Cauchy-Euler . . . . . . . . . . . . . . 107

$4.4 \quad$ Algumas Aplicações . . . . . . . . . . . . . . . . 109

4.4.1 Corpo em Queda Livre com Influência do Atrito . . . . . . . . . . . . 109

4.4.2 Aquecimento e Resfriamento de Prédios . . . . . . . . . . . . . . 111

4.4.3 Um Modelo para Detecção de Diabetes . . . . . . . . . . . . . . . . . 113

5 O MÉTODO DE APROXIMAÇÕES DE EULER E UMA APLICAÇÃO NA EDUCAÇÃO BÁSICA . . . . . . . . . . . . . . . . . 119

$5.1 \quad 0$ método de Euler . . . . . . . . . . . . . . 119

5.2 O Método de Euler Aperfeiçoado . . . . . . . . . . . . . . . 124

$5.3 \quad$ Uma Aplicação na Educação Básica . . . . . . . . . . . . . . . 130

5.3.1 A aplicação . . . . . . . . . . . . . . . . . . 130

5.3.2 Resultados . . . . . . . . . . . . . . . . 131

$5.3 .3 \quad$ Conclusão . . . . . . . . . . . . . . . . . . . 132

REFERÊNCIAS . . . . . . . . . . . . . . . . . . 133 


\section{1}

\section{INTRODUÇÃO}

Muito se fala em contextualização dos conteúdos ensinados, no caso de matemática, ao mesmo tempo em que há diversas possibilidades para contextualizar os conteúdos, há também o problema de gerar dúvidas, como "por que é deste jeito?" ou "por que se usa esse número e não o outro?". Um exemplo é quando se relaciona o conceito de funções exponenciais a populações usando a potência de base $e$ e não a base 2, questionamento esse feito por mim.

Para evitar esse tipo de dúvidas e até mesmo aprofundar mais no tema da contextualização, o ideal é que seja feita uma modelagem da situação que está sendo trabalhada no momento. Em muitos desses modelos, surgem equações envolvendo taxas de variações e, por consequência, as chamadas equações diferenciais.

O estudo das equações diferenciais foi iniciado no final do século $X V I I$, a partir dos estudos de Issac Newton e Gottfried Leibniz sobre o Cálculo Diferencial. Desde então, esses tipos de equações vêm atraindo a atenção de muitos matemáticos.

Atualmente, a teoria de equações diferenciais é considerada uma das áreas mais importantes, dinâmicas e aplicadas da matemática. Especificamente, é uma teoria que está em constante desenvolvimento e possui um estreito relacionamento com diversas outras áreas, pois constitui uma ferramenta importante para a modelagem de problemas concretos que surgem, por exemplo, em física, biologia, química, ciências da engenharia, economia, dentre outras.

O objetivo deste trabalho é o estudo de alguns conceitos, propriedades e aplicações da teoria de equações diferenciais ordinárias. Especificamente, iniciamos o estudo apresentando a definição de uma equação diferencial ordinária, alguns problemas concretos que podem ser modelados e estudados através de equações diferenciais ordinárias bem como um teorema de existência e unicidade de soluções. Além disso, estudamos alguns métodos de resolução para equações diferenciais ordinárias de primeira e segunda ordens e algumas aplicações relacionadas a esses métodos. Finalmente, apresentamos o estudo do método de Euler (um método numérico) para equações de primeira ordem, para que fosse possível uma aplicação do estudo de equações 
diferenciais com alunos do ensino médio.

O estudo dos resultados e aplicações acima citados são apresentados de forma detalhada, esperando que seja um material de fácil leitura e que, ao mesmo tempo, estimule o interesse do leitor num tipo de equação que está estreitamente relacionado ao nosso dia-a-dia.

Este trabalho é baseado, principalmente, em (BOYCE; DIPRIMA, 2006), (JUNIOR; LADEIRA, 2011), (JUNIOR, 2013), (NAGLE; SAFF; SNIDER, 2012) e está dividido em quatro capítulos. A seguir descrevemos, brevemente, os conteúdos abordados em cada um desses capítulos.

No Capítulo 2, apresentamos os conceitos básicos de equações diferenciais, como sua definição, alguns problemas concretos que podem ser modelados por equações diferenciais ordinárias e também um teorema de existência e unicidade de soluções para equações diferenciais de primeira ordem utilizando o método das iteradas de Picard, e com a demonstração baseada em (BRAUN, 1992) e (CHINCHIO, 2012).

No Capítulo 3, apresentamos métodos para encontrar soluções para alguns tipos de equações diferenciais ordinárias de primeira ordem e algumas aplicações envolvendo equações desse tipo como, por exemplo, a catenária, baseada em (FARIA, 2011) e (TEIXEIRA, 2012). Além disso, vimos dois tipos de equações que não são de primeira ordem, mas podem ser convertidas para esta forma.

No Capítulo 4, apresentamos métodos para encontrar soluções para equações diferenciais ordinárias lineares de segunda ordem e algumas aplicações envolvendo esse tipo de equação como, por exemplo, um modelo para detecção de diabetes, baseado em (BRAUN, 1992) e (CHINCHIO, 2012). Além disso, apresentamos a equação de Cauchy-Euler, uma equação mais geral do que as equações ordinárias de segunda ordem com coeficientes constantes, mas que pode ser tratada de maneira similar a essas.

Finalmente, no Capítulo 5, apresentamos o método de aproximações de Euler, que é um método numérico para obter aproximações de soluções de equações diferenciais ordinárias de primeira ordem e também o método de Euler aperfeiçoado, baseado em (NAGLE; SAFF; SNIDER, 2012). Além disso, apresentamos uma aplicação do ensino das equações diferenciais feita para alguns alunos do ensino médio utilizando os dois métodos acima para resolvê-las. E, por fim, apresentamos uma conclusão sobre a possibilidade de integrar o conceito de equações diferenciais ao ensino médio. 
CAPÍTULO

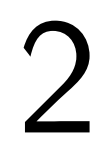

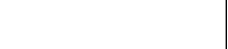

\section{EQUAÇÕES DIFERENCIAIS ORDINÁRIAS E UM TEOREMA DE EXISTÊNCIA E UNICIDADE}

A matemática se relaciona com diversos fenômenos da natureza e do universo, e muitos modelos matemáticos são desenvolvidos para auxiliar no entendimento desses fenômenos, os quais, geralmente, envolvem mudanças com o passar do tempo.

Considerando que a derivada $\frac{d y}{d t}=f^{\prime}(t)$ de uma função $f$ pode ser vista como a taxa na qual a quantidade $y=f(t)$ varia em relação à variável independente $t$, é natural esperar que os modelos acima citados gerem equações envolvendo uma função desconhecida e suas derivadas. Essas equações são chamadas de equações diferenciais e serão apresentadas e estudadas neste e nos próximos capítulos.

Especificamente, neste capítulo, estudamos conceitos importantes para uma classe de equações diferenciais bem como alguns problemas concretos que podem ser modelados por essas equações e um teorema de existência e unicidade de soluções para algumas dessas equações.

\subsection{Equações Diferenciais Ordinárias}

As equações diferenciais, em geral, são definidas da seguinte maneira.

Definição 1. Uma equação diferencial é uma equação que envolve uma função incógnita e suas derivadas.

A seguir temos alguns exemplos de equações diferenciais.

(1) $y^{\prime}(t)=3 t+2$ 
(2) $y^{\prime \prime}(t)+y^{\prime}(t)-2 y(t)=0$

(3) $t y^{\prime \prime}(t)+y^{\prime}(t)=4$

(4) $\left(y^{\prime \prime \prime}(t)\right)^{2}+\left(y^{\prime \prime}(t)\right)^{4}+t y(t)=y(t)$

(5) $y^{\prime \prime}(t)+2 y(t) y^{\prime}(t)+t y(t)=0$

(6) $\left(y^{\prime}(t)\right)^{3}-y(t)=0$

(7) $\frac{\partial z(t, y)}{\partial t}+\frac{\partial z(t, y)}{\partial y}=z(t, y)$

(8) $\frac{\partial^{2} z(t, y)}{\partial t^{2}}+\frac{\partial^{2} z(t, y)}{\partial y^{2}}=t+y$

Uma equação diferencial na qual a função incógnita depende apenas de uma variável, ou seja, só há uma variável independente, é denominada equação diferencial ordinária (EDO), e são exemplos de EDOs as equações de (1) à (6) acima. As equações desse tipo serão o foco deste trabalho.

Quando a função incógnita depender de mais de uma variável, ou seja, quando houver duas ou mais variáveis independentes, e a equação envolver derivadas parciais da função incógnita com relação a diferentes variáveis independentes, então a equação diferencial é denominada equação diferencial parcial (EDP). Por exemplo, as equações (7) e (8) acima são EDPs, pois em ambos os casos a função incógnita $z=z(t, y)$ depende das variáveis $t$ e $y$ e as equações apresentam derivadas de $z$ tanto em relação a $t$ quanto a $y$. Neste trabalho não trataremos de equações desse tipo.

Definição 2. A ordem de uma equação diferencial é a ordem da mais alta derivada que nela aparece.

Por exemplo, as equações (1), (6) e (7) são de primeira ordem, já as equações (2), (3), (5) e (8) são de segunda ordem e a equação (4) é de terceira ordem.

Definição 3. O grau de uma equação diferencial é o grau da derivada de mais alta ordem.

As equações (1), (2), (3), (5), (7), (8) são de primeiro grau. A equação (4) é de segundo grau, pois embora ela possua 4 como o expoente de $y^{\prime \prime}(t)$, o expoente que devemos analisar é o de $y^{\prime \prime \prime}(t)$, porque esta é a derivada de maior ordem. Já a equação (6) é de terceiro grau.

Definição 4. Uma solução de uma equação diferencial é uma função $y=y(t)$, que juntamente com suas derivadas, satisfaz essa equação. 
Exemplo 1. A função $y(t)=2 e^{t}$ é solução da equação diferencial ordinária $(1-t) y^{\prime \prime}+t y^{\prime}-y=0$, pois $y^{\prime}=2 e^{t}, y^{\prime \prime}=2 e^{t}$ e substituindo na equação temos

$$
(1-t)\left(2 e^{t}\right)+t\left(2 e^{t}\right)-\left(2 e^{t}\right)=\left(2 e^{t}\right)(1-t+t-1)=0 .
$$

Mais ainda, a função $z(t)=3 t$ também é solução da EDO dada, pois $z^{\prime}=3, z^{\prime \prime}=0$ e substituindo na equação temos

$$
(1-t) 0+t 3-3 t=0
$$

Finalmente, é interessante observar que se considerarmos a função $f(t)=y(t)+z(t)$, ou seja, $f(t)=2 e^{t}+3 t$, ela também é solução da EDO dada, pois $f^{\prime}=2 e^{t}+3, f^{\prime \prime}=2 e^{t}$ e fazendo a substituição na equação temos

$$
(1-t)\left(2 e^{t}\right)+t\left(2 e^{t}+3\right)-\left(2 e^{t}+3 t\right)=2 e^{t}-t 2 e^{t}+t 2 e^{t}+3 t-2 e^{t}-3 t=0 .
$$

Utilizando uma notação mais formal, podemos definir uma equação diferencial ordinária da seguinte forma.

Definição 5. Uma equação diferencial ordinária é uma equação da forma

$$
F\left(t, y(t), y^{\prime}(t), \ldots, y^{(n)}(t)\right)=0
$$

envolvendo uma função incógnita, $y=y(t)$, e suas derivadas.

Na teoria de Equações Diferenciais Ordinárias é comum representar a equação (2.1) da seguinte forma

$$
y^{(n)}(t)=f\left(t, y(t), y^{\prime}(t), \ldots, y^{(n-1)}(t)\right) .
$$

Observação 1. Para facilitar a notação, muitas vezes escreveremos $y=y(t)$. Neste caso, (2.2) fica

$$
y^{(n)}=f\left(t, y, y^{\prime}, \ldots, y^{(n-1)}\right)
$$

Como veremos mais adiante, é de grande utilidade classificar uma EDO como linear ou não linear. Portanto, a seguir apresentamos uma definição de linearidade e, mais para a frente, apresentaremos uma outra definição que será mais adequada para os nossos estudos.

Definição 6. Uma EDO é dita linear quando a variável dependente e suas derivadas aparecem somadas e elevadas ao grau 1 , da seguinte forma

$$
f_{n}(t) y^{(n)}+f_{n-1}(t) y^{(n-1)}+\ldots+f_{1}(t) y^{\prime}+f_{0}(t) y=g(t) .
$$

Caso contrário chamamos a EDO de não linear.

Assim, notamos que as equações (1), (2), (3) acima são lineares, enquanto (4), (5) e (6) são não lineares, pois tanto (4) quanto (6) apresentam termos elevados a graus diferentes de 1 , e (5) apresenta o termo $2 y \cdot y^{\prime}$, onde temos a variável dependente multiplicando uma de suas derivadas. 


\subsection{Problemas concretos envolvendo EDOs}

\subsubsection{Variação da Temperatura de um Corpo}

Sabemos que a taxa de variação da temperatura $T(t)$ de um corpo no instante $t$ é proporcional à diferença entre a temperatura do corpo e a temperatura do ambiente onde o corpo se encontra. Denotando por $T_{a}$ a temperatura do ambiente e considerando-a constante, essa situação pode ser descrita pela equação

$$
\frac{d T}{d t}=-K\left(T(t)-T_{a}\right), \quad K>0
$$

que pode ser reescrita como

$$
T^{\prime}(t)+K T(t)-K T_{a}=0
$$

onde $K$ é uma constante de proporcionalidade.

Note que o sinal - acompanhando $K$ na equação acima deve-se ao fato de que o calor flui da fonte quente para a fonte fria. Desse modo, quando a temperatura do corpo for maior que a temperatura do ambiente, ocorre que a temperatura do corpo cai (decresce), portanto a variação é negativa. Logo, como o fator $\left(T-T_{a}\right)$ é positivo, devemos multiplicá-lo por um valor negativo.

$$
T(t)>T_{a} \Rightarrow \text { o corpo perde calor } \Rightarrow T(t)-T(\tilde{t})<0, \text { se } t>\tilde{t} \Rightarrow \frac{d T}{d t}<0 .
$$

Por outro lado, quando a temperatura do corpo é menor que a temperatura do ambiente, ocorre que a temperatura do corpo aumenta (cresce), portanto a variação é positiva. Logo, como o fator $\left(T-T_{a}\right)$ é negativo, devemos multiplicá-lo por um valor negativo.

$$
T(t)<T_{a} \Rightarrow \text { o corpo ganha calor } \Rightarrow T(t)-T(\tilde{t})>0, \text { se } t>\tilde{t} \Rightarrow \frac{d T}{d t}>0 .
$$

\subsubsection{Decaimento Radioativo}

A taxa de desintegração (variação) de um elemento radioativo, em um dado instante, é proporcional à quantidade de elemento naquele instante. Tal situação pode ser descrita pela equação

$$
\frac{d Q}{d t}=-K Q, \quad K>0
$$

que equivale a

$$
Q^{\prime}(t)+K Q(t)=0,
$$

onde $Q(t)$ representa a quantidade do elemento no instante $t$. Como se trata de decaimento, ou seja, decrescimento, a quantidade do elemento presente em certo instante é menor que a quantidade presente em qualquer instante anterior, ou seja, $\frac{d Q}{d t}<0$ e, por outro lado, a quantidade $Q(t)$ é maior ou igual a zero. Por isso, o fator pelo qual multiplicamos $Q(t)$ deve ser negativo. 


\subsubsection{Corpo em Queda Livre com Influência do Atrito}

Um corpo em queda livre está sob influência da gravidade (agindo a favor do movimento) e da resistência do ar (agindo contra o movimento) de forma proporcional à velocidade do corpo. Tomando $h(t)$ como a altura do corpo, em relação ao solo, no instante $t$, obtemos pela $2^{a}$ lei de Newton $(F=m \cdot a)$ a equação do movimento como

$$
m \frac{d^{2} h}{d t^{2}}=m g-K \frac{d h}{d t}, \quad K>0,
$$

que é equivalente a

$$
m h^{\prime \prime}(t)=m g-K h^{\prime}(t),
$$

onde $m$ representa a massa do corpo, $g(>0)$ a aceleração da gravidade e $K(>0)$ a contatante de resistência do ar.

Chegamos a esse resultado, pois, da física, temos que a força resultante $F$ de um corpo é igual à soma das forças que atuam no corpo. Assim sendo, a força resultante equivale a $m \cdot a$, onde a aceleração é obtida por meio da derivada da velocidade em relação ao tempo, e a velocidade, por sua vez, da derivada da função espaço (neste caso, $h(t)$ ) em relação ao tempo. Ou seja, a aceleração corresponde a segunda derivada da função espaço em relação ao tempo $\left(a=h^{\prime \prime}(t)\right)$. Por outro lado, a soma das forças que atuam sobre o corpo corresponde à gravidade subtraída a resistência do ar, ou seja, $m \cdot g-K h^{\prime}(t)$, assim chegamos à forma final da equação.

\subsubsection{Modelos Populacionais}

\subsubsection{Modelo de Malthus}

Suponhamos que as taxas de natalidade e mortalidade de uma população sejam proporcionais à população presente, suposta maior que zero. Assim, a variação populacional corresponde à diferença entre a taxa de natalidade e a taxa de mortalidade. Podemos descrever esta situação do seguinte modo,

$$
\frac{d p}{d t}=k_{1} p(t)-k_{2} p(t)=\left(k_{1}-k_{2}\right) p(t)
$$

ou seja,

$$
p^{\prime}(t)=k p(t)
$$

onde $p(t)$ representa a população no instante $t$, sendo $k_{1}$ a constante de proporcionalidade da taxa de natalidade, $k_{2}$ a constante de proporcionalidade da taxa de mortalidade e $k=k_{1}-k_{2}$.

Note que se $k_{1}>k_{2}$, então $k>0$ e a população cresce, mas se $k_{1}<k_{2}$, então $k<0$ e a população decresce.

\subsubsection{Modelo de Verhulst}

No modelo de Malthus considera-se apenas a mortalidade natural da população. Entretanto, sabemos que existem muitos outros fatores que afetam a mortalidade, tais como, escassez 
de alimento e água, alguns tipos de doenças, entre outros. O modelo de Verhulst, também conhecido como modelo logístico, considera fatores limitantes do ambiente, e pode ser expresso na seguinte forma

$$
p^{\prime}(t)=k p(t)\left(1-\frac{p(t)}{C}\right),
$$

onde $p(t)$ representa a população no instante $t, k$ a taxa de crescimento na falta de fator limitante e $C$ é a capacidade de carga populacional suportada pelo ambiente em que a população vive, ou nível de saturação.

Especificamente, para que, no modelo de Malthus, a taxa de crescimento realmente dependa da população devemos substituir a constante $k$ por uma função $f(p)$, tal que, quando a população for pequena, ela seja próxima de $k>0$ (para uma população devidamente pequena as condições do ambiente não serão um fator limitante), decresça conforme a população aumente e $f(p)<0$ quando a população for suficientemente grande. A função mais simples com tais características é $f(p)=k-a p$, onde $a$ é uma constante positiva. Substituindo a constante $k$ por esta função no modelo de Malthus, temos que a taxa de variação populacional para esse modelo pode ser dada por

$$
p^{\prime}=(k-a p) p .
$$

Colocando $k$ em evidência e tomando $\frac{k}{C}=a$ temos

$$
\frac{d p}{d t}=k p\left(1-\frac{p}{C}\right)
$$

Podemos ver que a equação acima comporta-se como o esperado, ou seja, uma vez que estamos interessados em $p(t)>0$ temos que

a) $p(t)>C \Rightarrow \frac{p(t)}{C}>1 \Rightarrow 1-\frac{p(t)}{C}<0 \Rightarrow p^{\prime}(t)<0$, o que representa um decrescimento da população.

b) $0<p(t)<C \Rightarrow 0<\frac{p(t)}{C}<1 \Rightarrow 1-\frac{p(t)}{C}>0 \Rightarrow p^{\prime}(t)>0$, o que representa um crescimento da população.

Mais ainda, estes fatos justificam dizer que $C$ é a capacidade de carga populacional suportada pelo ambiente ou nível de saturação.

\subsubsection{Modelo Presa-predador}

A relação predatória entre duas espécies também pode ser expressa por uma equação diferencial ordinária e para tal, devemos considerar algumas hipóteses sobre a população de presas $p_{x}(t)$, e a população de predadores $p_{y}(t)$. Então,

- Na ausência de predador, a população de presas comporta-se de forma malthusiana, ou seja, varia de acordo com a população atual e, assim, $\frac{d p_{x}}{d t}=a p_{x}(t)$, onde $a>0$ representa a taxa de crescimento das presas. 
- Na ausência de presas, a população de predador diminui e, desse modo, $\frac{d p_{y}}{d t}=-b p_{y}(t)$, onde $b>0$ representa a taxa de mortalidade dos predadores.

- O número de interações entre presa e predador é proporcional ao produto das duas espécies, onde o crescimento da população de predadores é estimulado e o da de presas é inibido. Dessa forma, a taxa de variação da população de presas é afetada por um termo da forma $-C p_{x} p_{y}$ e a de predadores por um termo da forma $K p_{x} p_{y}$, onde $C, K>0$. Portanto, temos que

$$
\begin{gathered}
\frac{d p_{x}}{d t}=a p_{x}(t)-C p_{x}(t) p_{y}(t) \\
\frac{d p_{y}}{d t}=-b p_{y}(t)+K p_{x}(t) p_{y}(t) .
\end{gathered}
$$

Por meio desse modelo temos que se a população de presas aumenta, então a população de predadores aumenta, mas se a população de predadores aumentar excessivamente, a população de presas diminui em mesma proporção, e a população de predadores é forçada a diminuir.

\subsection{Existência e Unicidade de Soluções}

Note que para estudarmos as propriedades de soluções de uma EDO, precisamos primeiro ter informações sobre a existência dessas soluções. Logo, o objetivo desta seção é apresentar um resultado de existência e unicidade de solução para uma EDO de primeira ordem. Para isso, precisamos ,inicialmente, introduzir alguns conceitos e definições.

Para começar, consideremos o seguinte exemplo.

Exemplo 2. Se um isótopo radioativo tem constante de desintegração equivalente a 0,1733 ao dia, temos que sua taxa de variação pode ser descrita pela seguinte EDO

$$
Q^{\prime}(t)+0,1733 Q(t)=0 .
$$

Mais ainda, se a quantidade do isótopo presente em algum momento for conhecido, por exemplo, se a quantidade inicial do isótopo for de 200 gramas, podemos descrever o problema a ser resolvido da seguinte forma

$$
\left\{\begin{array}{l}
Q^{\prime}(t)=-0,1733 Q(t) \\
Q(0)=200
\end{array}\right.
$$

O problema que surgiu após o conhecimento da quantidade inicial de isótopo é o que chamamos de problema de valor inicial. Formalmente, temos a seguinte definição.

Definição 7. Um Problema de Valor Inicial (P.V.I.) consiste em uma equação diferencial acompanhada do valor da função incógnita em um determinado ponto, a condição inicial. 
Assim, um P.V.I. para uma EDO de primeira ordem pode ser descrito na forma

$$
\left\{\begin{array}{l}
y^{\prime}(t)=f(t, y(t)), \\
y\left(t_{0}\right)=y_{0},
\end{array}\right.
$$

onde $f$ é uma função definida num aberto $A$ de $\mathbb{R}^{2}, \operatorname{com}\left(t_{0}, y_{0}\right) \in A$.

Note agora que, se estivermos no caso particular em que $f:[a, b] \rightarrow \mathbb{R}$ é uma função contínua, segue do Teorema Fundamental do Cálculo, que a função

$$
F(t)=\int_{a}^{t} f(s) d s, \quad a \leqslant t \leqslant b
$$

é diferenciável em $(a, b)$ e $F^{\prime}(t)=f(t)$ para todo $t \in(a, b)$. Logo, $F(t)$ é uma solução da EDO de primeira ordem

$$
y^{\prime}(t)=f(t), \quad a<t<b .
$$

Mais ainda, como $F(a)=0$, temos que $F(t)$ é uma solução do P.V.I.

$$
\left\{\begin{array}{l}
y^{\prime}(t)=f(t), \quad a<t<b, \\
y(a)=0 .
\end{array}\right.
$$

Mas podemos questionar se $F(t)$ é a única solução para o P.V.I. acima. Neste caso, a solução é única, pois se $G(t)$ é uma outra solução, então

$$
G^{\prime}(t)=f(t)=F^{\prime}(t) \Rightarrow(F-G)^{\prime}(t)=0 \Rightarrow(F-G)(t)=c, \quad t \in(a, b),
$$

onde $c$ é uma constante. Como $F-G$ é uma função contínua em $[a, b]$, podemos concluir que

$$
(F-G)(t)=c, \forall t \in[a, b] .
$$

Por outro lado, temos que

$$
(F-G)(a)=F(a)-G(a)=0-0=0 .
$$

Desse modo, $c=0$ e $F(t)=G(t)$ para todo $t \in[a, b]$.

Entretanto, existem casos em que o P.V.I. dado não apresenta unicidade da solução. Considere, por exemplo, o P.V.I.

$$
\left\{\begin{array}{l}
y^{\prime}(t)=|y(t)|^{\frac{1}{2}}, \quad t \in \mathbb{R} \\
y(0)=0
\end{array}\right.
$$

É fácil verificar que ambas as funções $y_{1}(t) \equiv 0 \mathrm{e}$

$$
y_{2}(t)=\left\{\begin{array}{l}
\frac{t^{2}}{4}, \quad t \geqslant 0, \\
\frac{-t^{2}}{4}, \quad t<0
\end{array}\right.
$$

são soluções do P.V.I. (2.5). Assim, o P.V.I. (2.5) possui pelo menos duas soluções.

Desse modo, dado o P.V.I. (2.4) surgem as seguintes questões: 
1. Como sabemos se o P.V.I. (2.4) possui solução?

2. Caso exista uma solução para (2.4), como saber se ela é única?

O teorema a seguir nos ajuda responder estas questões.

Teorema 1. (Existência e Unicidade Local) Suponha que $f$ e $\frac{\partial f}{\partial y}$ sejam funções contínuas no retângulo $R=\left\{(t, y) \mid t_{0} \leqslant t \leqslant t_{0}+a\right.$ e $\left.\left|y-y_{0}\right| \leqslant b\right\} \subset A$, onde $a, b \in \mathbb{R}^{+}$. Sejam ainda $M=\max _{(t, y) \in R}|f(t, y)|$ e $\alpha=\min \left\{a, \frac{b}{M}\right\}$. Então, o P.V.I. (2.4) possui uma única solução $y=y(t)$ no intervalo $\left[t_{0}, t_{0}+\alpha\right]$.

Antes de provar este teorema, introduzimos o seguinte resultado.

Proposição 1. Seja $\alpha>0$ tal que $(t, y(t)) \in A$ para todo $t \in\left[t_{0}, t_{0}+\alpha\right]$. Suponha que $f$ seja contínua em $A$ e que $y$ seja contínua em $\left[t_{0}, t_{0}+\alpha\right]$. Então, $y=y(t)$ é solução de (2.4) se, e somente se,

$$
y(t)=y_{0}+\int_{t_{0}}^{t} f(s, y(s)) d s, t \in\left[t_{0}, t_{0}+\alpha\right]
$$

Demonstração. Seja y uma solução de (2.4). Então, integrando ambos os membros da equação $y^{\prime}=f(t, y)$ em relação a $t$ temos que

$$
\int_{t_{0}}^{t} y^{\prime}(s) d s=\int_{t_{0}}^{t} f(s, y(s)) d s
$$

que, pelo Teorema Fundamental do Cálculo, resulta em

$$
y(t)-y\left(t_{0}\right)=\int_{t_{0}}^{t} f(s, y(s)) d s, \quad t \in\left[t_{0}, t_{0}+\alpha\right]
$$

ou seja,

$$
y(t)=y_{0}+\int_{t_{0}}^{t} f(s, y(s)) d s, \quad t \in\left[t_{0}, t_{0}+\alpha\right]
$$

Reciprocamente, se

$$
y(t)=y_{0}+\int_{t_{0}}^{t} f(s, y(s)) d s, \quad t \in\left[t_{0}, t_{0}+\alpha\right]
$$

como $f$ e $y$ são contínuas, quando derivamos em relação a $t$ temos que

$$
\begin{aligned}
y^{\prime}(t) & =\frac{d}{d t}\left(\int_{t_{0}}^{t} f(s, y(s)) d s\right) \\
& =f(t, y(t)) .
\end{aligned}
$$

Mais ainda, é claro que $y\left(t_{0}\right)=y_{0}$. Portanto, $y=y(t)$ é solução do P.V.I. (2.4) no intervalo $\left[t_{0}, t_{0}+\alpha\right]$.

Observação 2. A equação (2.6) é chamada Equação Integral associada ao P.V.I. (2.4) 
A ideia da demonstração que apresentaremos para o Teorema 1 é de construir uma sequência de funções $\left(y_{n}(t)\right)_{n}$ que seja convergente para uma função $y(t)$ no intervalo $\left[t_{0}, t_{0}+\alpha\right]$ que seja uma solução contínua de (2.6) e, portanto, do P.V.I. (2.4). Em seguida mostraremos que a solução encontrada é única.

Supondo que as hipóteses Teorema 1 sejam verificadas, dividiremos sua demonstração nas 4 etapas a seguir.

\section{Construção da sequência de funções}

Começamos definindo $y_{0}(t)=y_{0}$. Para verificar se $y_{0}(t)$ é solução de (2.6) calculamos

$$
y_{1}(t):=y_{0}+\int_{t_{0}}^{t} f\left(s, y_{0}(s)\right) d s .
$$

Se $y_{1}(t)=y_{0}, t \in\left[t_{0}, t_{0}+\alpha\right]$, então $y(t)=y_{0}$ é uma solução de (2.6). Caso contrário, verificamos se $y_{1}(t)$ é solução de (2.6) calculando

$$
y_{2}(t):=y_{0}+\int_{t_{0}}^{t} f\left(s, y_{1}(s)\right) d s
$$

Se $y_{2}(t)=y_{1}(t), t \in\left[t_{0}, t_{0}+\alpha\right]$, então $y(t)=y_{1}(t)$ é solução de (2.6). Caso contrário, verificamos se $y_{2}(t)$ é solução de (2.6) calculando

$$
y_{3}(t):=y_{0}+\int_{t_{0}}^{t} f\left(s, y_{2}(s)\right) d s .
$$

Se $y_{3}(t)=y_{2}(t), t \in\left[t_{0}, t_{0}+\alpha\right]$, então $y(t)=y_{2}(t)$ é solução de (2.6). Caso contrário, verificamos a próxima iteração e assim por diante. Desse modo, definimos uma sequência de funções $\left(y_{n}(t)\right)_{n}$, onde

$$
y_{n}(t)=y_{0}+\int_{t_{0}}^{t} f\left(s, y_{n-1}(s)\right) d s
$$

Definição 8. Chamamos a sequência de funções

$$
y_{n}(t)=y_{0}+\int_{t_{0}}^{t} f\left(s, y_{n-1}(s)\right) d s, \quad n \in \mathbb{N},
$$

de Iteradas de Picard do P.V.I. (2.4).

Exemplo 3. Calculemos as iteradas de Picard do P.V.I.

$$
\left\{\begin{array}{l}
y^{\prime}=y, \\
y(0)=1
\end{array}\right.
$$

Primeiramente, note que a equação integral correspondente ao problema dado é

$$
y(t)=y(0)+\int_{0}^{t} y(s) d s=1+\int_{0}^{t} y(s) d s .
$$


Então, fazendo $y_{0}(t)=1$ temos que

$$
\begin{aligned}
& y_{1}(t)=1+\int_{0}^{t} 1 d s=1+t, \\
& y_{2}(t)=1+\int_{0}^{t}(1+s) d s=1+t+\frac{t^{2}}{2 !}, \\
& y_{3}(t)=1+\int_{0}^{t}\left(1+s+\frac{s^{2}}{2}\right) d s=1+t+\frac{t^{2}}{2 !}+\frac{t^{3}}{3 !} .
\end{aligned}
$$

Continuando o processo acima podemos ver que

$$
y_{n}(t)=1+\int_{0}^{t} y_{n-1}(s) d s=1+t+\frac{t^{2}}{2 !}+\cdots+\frac{t^{n}}{n !}=\sum_{i=0}^{n} \frac{t^{i}}{i !} .
$$

Além disso, é bem conhecido que

$$
\lim _{n \rightarrow \infty} y_{n}(t)=\sum_{i=0}^{\infty} \frac{t^{i}}{i !}=e^{t}
$$

que é solução do P.V.I. dado.

O exemplo acima é uma motivação para a próxima etapa em que mostramos que as Iteradas de Picard sempre definem uma sequência que converge para uma solução do P.V.I. (2.4).

\section{Convergência das Iteradas de Picard}

É importante notar que, em geral, as soluções de uma EDO podem não existir para todos os pontos $t$ do domínio. Logo, é natural pensar que as Iteradas de Picard podem não convergir para todo $t$ do seu domínio, caso elas convirjam, realmente, para uma solução do P.V.I. (2.4). Assim, para descobrir onde as Iteradas de Picard convergem, tentaremos, inicialmente, encontrar um intervalo $I$ em que $\left(y_{n}(t)\right)_{n}$ seja uniformemente limitada, ou seja, $\left|y_{n}(t)\right| \leqslant K$ para todo $n \in \mathbb{N}$ e $t \in I$, onde $K$ é uma constante fixada.

Em outras palavras, buscaremos uma região retangular $R$ que contenha os gráficos de todas as iteradas de Picard. O lema a seguir mostra como encontrar esta região.

Lema 1. Sejam $a, b \in \mathbb{R}^{+}$tais que $R=\left\{(t, y) \mid t_{0} \leqslant t \leqslant t_{0}+a\right.$ e $\left.\left|y-y_{0}\right| \leqslant b\right\} \subset A$ e $f$ seja contínua em $R$. Se $M=\max _{(t, y) \in R}|f(t, y)|$ e $\alpha=\min \left\{a, \frac{b}{M}\right\}$, então

$$
\left|y_{n}(t)-y_{0}\right| \leqslant M\left(t-t_{0}\right), \forall t \in\left[t_{0}, t_{0}+\alpha\right] .
$$

Demonstração. Provaremos o resultado por indução sobre $n$. Para $n=0$ é claro que (2.7) vale, pois

$$
\left|y_{0}(t)-y_{0}\right|=0
$$

para todo $t$. Agora, suponha que (2.7) vale para $n-1$, ou seja,

$$
\left|y_{n-1}(t)-y_{0}\right| \leqslant M\left(t-t_{0}\right), \forall t \in\left[t_{0}, t_{0}+\alpha\right]
$$


e note que

$$
\begin{aligned}
\left|y_{n}(t)-y_{0}\right| & =\left|y_{0}+\int_{t_{0}}^{t} f\left(s, y_{n-1}(s)\right) d s-y_{0}\right| \\
& =\left|\int_{t_{0}}^{t} f\left(s, y_{n-1}(s)\right) d s\right| \\
& \leqslant \int_{t_{0}}^{t}\left|f\left(s, y_{n-1}(s)\right)\right| d s .
\end{aligned}
$$

Mas, para todo $t \in\left[t_{0}, t_{0}+\alpha\right]$,

$$
\left|y_{n-1}(t)-y_{0}\right| \leqslant M\left(t-t_{0}\right) \Leftrightarrow-M\left(t-t_{0}\right)+y_{0} \leqslant y_{n-1}(t) \leqslant M\left(t-t_{0}\right)+y_{0} .
$$

Assim, definindo as retas

$$
\begin{aligned}
& r: y(t)=-M\left(t-t_{0}\right)+y_{0}, \quad t \in \mathbb{R}, \\
& s: y(t)=M\left(t-t_{0}\right)+y_{0}, \quad t \in \mathbb{R},
\end{aligned}
$$

vemos que $y_{n-1}(t)$ fica entre $r$ e $s$ quando $t \in\left[t_{0}, t_{0}+\alpha\right]$. Mais ainda, aplicando essas retas no ponto $t_{0}+\alpha$ e lembrando que $\alpha=\min \left\{a, \frac{b}{M}\right\}$ temos que

$$
\begin{aligned}
& r: y\left(t_{0}+\alpha\right)=-M\left(t_{0}+\alpha-t_{0}\right)+y_{0}=-M \alpha+y_{0} \geqslant y_{0}-b \\
& s: y\left(t_{0}+\alpha\right)=M\left(t_{0}+\alpha-t_{0}\right)+y_{0}=M \alpha+y_{0} \leqslant y_{0}+b .
\end{aligned}
$$

Portanto, $\left(t, y_{n-1}(t)\right) \in R$, para todo $t \in\left[t_{0}, t_{0}+\alpha\right]$, como mostra a Figura 1 a seguir. Assim,

$$
\left|f\left(t, y_{n-1}(t)\right)\right| \leqslant M, \quad \forall t \in\left[t_{0}, t_{0}+\alpha\right]
$$

Daí segue de (2.8) que

$$
\left|y_{n}(t)-y_{0}\right| \leqslant \int_{t_{0}}^{t}\left|f\left(s, y_{n-1}(s)\right)\right| d s \leqslant M\left(t-t_{0}\right), \quad \forall t \in\left[t_{0}, t_{0}+\alpha\right] .
$$

Note que, usando o mesmo raciocínio na demonstração do Lema 1, vemos que o resultado deste Lema afirma que o gráfico de $y_{n}(t)$ está entre as retas $r$ e $s$, e que, portanto, $\left(t, y_{n}(t)\right) \in R$, para todo $t \in\left[t_{0}, t_{0}+\alpha\right]$ e todo $n=0,1,2, \ldots$ Este significado geométrico está apresentado na Figura 1 a seguir. 
Figura 1 - Retas $r$ e $s$ delimitando a região em que se encontram os termos da sequência de Picard

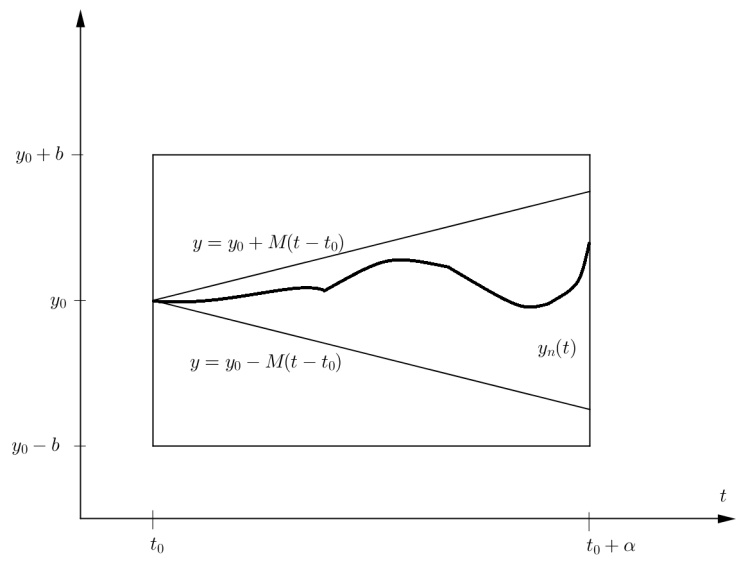

(a) $\alpha=a$

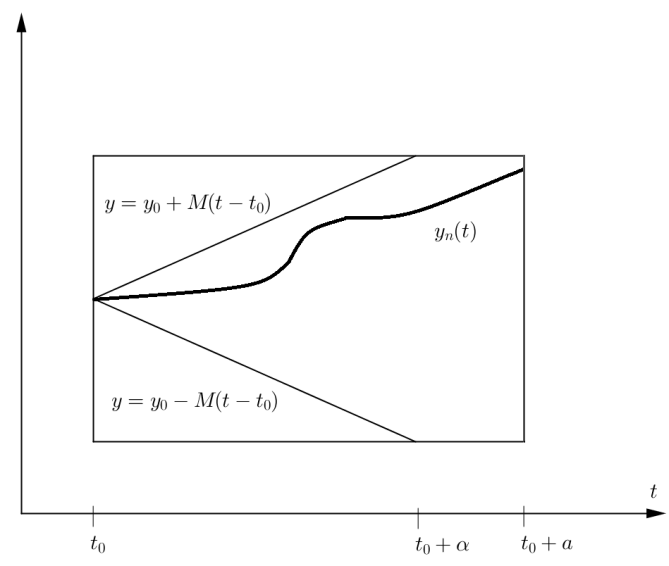

(b) $\alpha=\frac{b}{M}$

Fonte: Elaborada pelo autor.

Proposição 2. Se $\frac{\partial f}{\partial y}$ existe e é contínua em $R$, então a sequência $\left(y_{n}(t)\right)_{n}$ definida pelas Iteradas de Picard, converge uniformemente no intervalo $\left[t_{0}, t_{0}+\alpha\right]$. Ou seja, existe uma função $y(\cdot)$ tal que $y_{n}(t) \rightarrow y(t)$ para todo $t \in\left[t_{0}, t_{0}+\alpha\right]$.

Demonstração. Primeiramente, vamos reduzir o problema de mostrar que a sequência de funções $\left(y_{n}(t)\right)_{n}$ converge para o problema de mostrar que uma determinada série infinita converge. Para isso, escrevemos $y_{n}(t)$ como

$$
y_{n}(t)=y_{0}(t)+\left[y_{1}(t)-y_{0}(t)\right]+\left[y_{2}(t)-y_{1}(t)\right]+\ldots+\left[y_{n-1}(t)-y_{n-2}(t)\right]+\left[y_{n}(t)-y_{n-1}(t)\right],
$$

ou seja,

$$
y_{n}(t)=y_{0}+\sum_{k=1}^{n}\left[y_{k}(t)-y_{k-1}(t)\right]
$$

Assim, $\left(y_{n}(t)\right)_{n}$ converge uniformemente em $\left[t_{0}, t_{0}+\alpha\right]$ se, e somente se, $\sum_{k=1}^{\infty}\left[y_{k}(t)-y_{k-1}(t)\right]$ converge uniformemente em $\left[t_{0}, t_{0}+\alpha\right]$.

Mostraremos a seguir que a série $\sum_{k=1}^{\infty}\left[y_{k}(t)-y_{k-1}(t)\right]$ converge uniformemente no intervalo $\left[t_{0}, t_{0}+\alpha\right]$. Para isso, note primeiro que

$$
\begin{aligned}
\left|y_{k}(t)-y_{k-1}(t)\right| & =\left|y_{0}+\int_{t_{0}}^{t} f\left(s, y_{k-1}(s)\right) d s-y_{0}-\int_{t_{0}}^{t} f\left(s, y_{k-2}(s)\right) d s\right| \\
& =\left|\int_{t_{0}}^{t} f\left(s, y_{k-1}(s)\right) d s-\int_{t_{0}}^{t} f\left(s, y_{k-2}(s)\right) d s\right| \\
& =\left|\int_{t_{0}}^{t}\left[f\left(s, y_{k-1}(s)\right)-f\left(s, y_{k-2}(s)\right)\right] d s\right| \\
& \leqslant \int_{t_{0}}^{t}\left|f\left(s, y_{k-1}(s)\right)-f\left(s, y_{k-2}(s)\right)\right| d s .
\end{aligned}
$$


Agora, aplicando o Teorema do Valor Médio para funções de uma variável, ou seja, se $f$ é contínua em $[a, b]$ e derivável em $(a, b)$, então existe $c \in(a, b)$ tal que $f(a)-f(b)=f^{\prime}(c)(b-a)$, na segunda componente de $f$, obtemos que

$$
\int_{t_{0}}^{t}\left|f\left(s, y_{k-1}(s)\right)-f\left(s, y_{k-2}(s)\right)\right| d s=\int_{t_{0}}^{t}\left|\frac{\partial f(s, \xi(s))}{\partial y}\right|\left|y_{k-1}(s)-y_{k-2}(s)\right| d s,
$$

onde $\xi(s)$ é um valor entre $y_{k-1}(s)$ e $y_{k-2}(s)$. Logo, definindo

$$
L=\max _{(t, y) \in R}\left|\frac{\partial f(t, y)}{\partial y}\right|
$$

e notando que $(s, \xi(s)) \in R$ para cada $s \in\left[t_{0}, t_{0}+\alpha\right]$, temos que

$$
\left|y_{k}(t)-y_{k-1}(t)\right| \leqslant \int_{t_{0}}^{t}\left|\frac{\partial f(s, \xi(s))}{\partial y}\right|\left|y_{k-1}(s)-y_{k-2}(s)\right| d s \leqslant L \int_{t_{0}}^{t}\left|y_{k-1}(s)-y_{k-2}(s)\right| d s
$$

para todo $t_{0} \leqslant t \leqslant t_{0}+\alpha$. Ou seja, temos

$$
\left|y_{k}(t)-y_{k-1}(t)\right| \leqslant L \int_{t_{0}}^{t}\left|y_{k-1}(s)-y_{k-2}(s)\right| d s, \quad t_{0} \leqslant t \leqslant t_{0}+\alpha .
$$

Logo, aplicando a equação (2.9) para $k=2$, segue do Lema 1 que

$$
\left|y_{2}(t)-y_{1}(t)\right| \leqslant L \int_{t_{0}}^{t}\left|y_{1}(s)-y_{0}(s)\right| d s \leqslant L \int_{t_{0}}^{t} M\left(s-t_{0}\right) d s=M L \frac{\left(t-t_{0}\right)^{2}}{2},
$$

o que implica, para $k=3$, que

$$
\left|y_{3}(t)-y_{2}(t)\right| \leqslant L \int_{t_{0}}^{t}\left|y_{2}(s)-y_{1}(s)\right| d s \leqslant M L^{2} \int_{t_{0}}^{t} \frac{\left(s-t_{0}\right)^{2}}{2} d s=M L^{2} \frac{\left(t-t_{0}\right)^{3}}{3 !} .
$$

Por indução, podemos concluir que para todo $t_{0} \leqslant t \leqslant t_{0}+\alpha$

$$
\begin{aligned}
{\left[y_{k}(t)-y_{k-1}(t)\right] } & \leqslant\left|y_{k}(t)-y_{k-1}(t)\right| \\
& \leqslant \frac{M L^{k-1}\left(t-t_{0}\right)^{k}}{k !} \\
& \leqslant \frac{M L^{k-1} \alpha^{k}}{k !} \\
& =\frac{M}{L} \frac{(\alpha L)^{k}}{k !} .
\end{aligned}
$$

Agora, a série $\sum_{k=1}^{\infty} \frac{M}{L} \frac{(\alpha L)^{k}}{k !}$ converge, pois

$$
\sum_{k=1}^{\infty} \frac{M}{L} \frac{(\alpha L)^{k}}{k !}=\frac{M}{L} \sum_{k=1}^{\infty} \frac{(\alpha L)^{k}}{k !}=\frac{M}{L}\left(\sum_{k=0}^{\infty} \frac{(\alpha L)^{k}}{k !}-1\right)=\frac{M}{L}\left(e^{\alpha L}-1\right) .
$$

Logo, pelo teste da comparação, a série $\sum_{k=1}^{\infty}\left[y_{k}(t)-y_{k-1}(t)\right]$ converge. Mais ainda, a série converge uniformemente em $\left[t_{0}, t_{0}+\alpha\right]$. Logo, $\left(y_{n}(t)\right)_{n}$ converge uniformemente no intervalo $\left[t_{0}, t_{0}+\alpha\right]$. Então, existe uma função $y(\cdot)$ tal que $y_{n}(t) \rightarrow y(t)$, para todo $t \in\left[t_{0}, t_{0}+\alpha\right]$. 


\section{A existência de solução}

Proposição 3. A função $y(\cdot)$ da Proposição 2 é solução do P.V.I. (2.4) em $\left[t_{0}, t_{0}+\alpha\right]$.

Demonstração. Para cada $t \in\left[t_{0}, t_{0}+\alpha\right]$, sabemos que $y_{n}(t)=y_{0}+\int_{t_{0}}^{t} f\left(s, y_{n-1}(s)\right) d s$. Logo, aplicando o limite quando $n \rightarrow \infty$ obtemos que

$$
\lim _{n \rightarrow \infty} y_{n}(t)=\lim _{n \rightarrow \infty}\left(y_{0}+\int_{t_{0}}^{t} f\left(s, y_{n-1}(s)\right) d s\right)
$$

o que resulta em

$$
y(t)=y_{0}+\lim _{n \rightarrow \infty} \int_{t_{0}}^{t} f\left(s, y_{n-1}(s)\right) d s .
$$

Então, basta mostrar que

$$
\lim _{n \rightarrow \infty} \int_{t_{0}}^{t} f\left(s, y_{n-1}(s)\right) d s=\int_{t_{0}}^{t} f(s, y(s)) d s .
$$

Agora, como na Proposição 2, usando o Teorema do Valor Médio segue que

$$
\begin{aligned}
\left|\int_{t_{0}}^{t} f(s, y(s)) d s-\int_{t_{0}}^{t} f\left(s, y_{k}(s)\right) d s\right| & \leqslant \int_{t_{0}}^{t}\left|f(s, y(s))-f\left(s, y_{k}(s)\right)\right| d s \\
& =\int_{t_{0}}^{t}\left|\frac{\partial f(s, \xi(s))}{\partial y}\right|\left|y(s)-y_{k}(s)\right| d s \\
& \leqslant L \int_{t_{0}}^{t}\left|y(s)-y_{k}(s)\right| d s .
\end{aligned}
$$

Mas observe que

$$
y(s)-y_{k}(s)=\sum_{j=k+1}^{\infty}\left[y_{j}(s)-y_{j-1}(s)\right],
$$

o que implica, como na Proposição 2, que

$$
\begin{aligned}
\left|y(s)-y_{k}(s)\right| & =\left|\sum_{j=k+1}^{\infty}\left[y_{j}(s)-y_{j-1}(s)\right]\right| \\
& \leqslant \sum_{j=k+1}^{\infty}\left|y_{j}(s)-y_{j-1}(s)\right| \\
& \leqslant \frac{M}{L} \sum_{j=k+1}^{\infty} \frac{(L \alpha)^{j}}{j !} .
\end{aligned}
$$

Logo,

$$
\left|\int_{t_{0}}^{t} f\left(s, y_{k}(s)\right)-\int_{t_{0}}^{t} f(s, y(s))\right| \leqslant M \sum_{j=k+1}^{\infty} \frac{(\alpha L)^{j}}{j !} \int_{t_{0}}^{t} d s \leqslant M \alpha \sum_{j=k+1}^{\infty} \frac{(\alpha L)^{j}}{j !} .
$$

Este somatório tende a zero quando $k$ tende ao infinito, uma vez que se trata da parte final da expansão da série de Taylor da função $e^{\alpha L}$. Desse modo, concluímos que

$$
\lim _{n \rightarrow \infty} \int_{t_{0}}^{t} f\left(s, y_{n}(s)\right) d s=\int_{t_{0}}^{t} f(s, y(s)) d s .
$$


Desse modo,

$$
y(t)=y_{0}+\int_{t_{0}}^{t} f(s, y(s)) d s,
$$

e, portanto, $y(\cdot)$ é solução de (2.6).

Mostremos agora que $y(\cdot)$ é contínua em $\left[t_{0}, t_{0}+\alpha\right]$. Para isso, devemos mostrar que, para $t \in\left[t_{0}, t_{0}+\alpha\right]$ e $h$ suficientemente pequeno tal que $t+h \in\left[t_{0}, t_{0}+\alpha\right]$, dado $\varepsilon>0$, existe $\delta>0$ tal que

$$
|y(t+h)-y(t)|<\varepsilon
$$

para $|h|<\delta$. Não podemos comparar $y(t+h) \operatorname{com} y(t)$ diretamente, pois não conhecemos $y(t)$ explicitamente. Então, escolhemos $n_{0}$ suficientemente grande de modo que $\frac{M}{L} \sum_{j=n_{0}+1}^{\infty} \frac{(\alpha L)^{j}}{j !}<\frac{\varepsilon}{3}$. Agora, observando que

$$
y(t+h)-y(t)=\left[y(t+h)-y_{n_{0}}(t+h)\right]+\left[y_{n_{0}}(t+h)-y_{n_{0}}(t)\right]+\left[y_{n_{0}}(t)-y(t)\right],
$$

e prosseguindo como em (2.11) temos que

$$
\left|y(t+h)-y_{n_{0}}(t+h)\right|<\frac{\varepsilon}{3} \quad \text { e } \quad\left|y_{n_{0}}(t)-y(t)\right|<\frac{\varepsilon}{3} .
$$

Por outro lado, por construção, $y_{n_{0}}$ é uma função contínua. Logo, existe $\delta>0$ tal que,

$$
\left|y_{n_{0}}(t+h)-y_{n_{0}}(t)\right|<\frac{\varepsilon}{3},
$$

para $|h|<\delta$. Consequentemente, para $|h|<\delta$ concluímos que

$$
\begin{aligned}
|y(t+h)-y(t)| & \leqslant\left|y(t+h)-y_{n_{0}}(t+h)\right|+\left|y_{n_{0}}(t+h)-y_{n_{0}}(t)\right|+\left|y_{n_{0}}(t)-y(t)\right| \\
& <\frac{\varepsilon}{3}+\frac{\varepsilon}{3}+\frac{\varepsilon}{3}=\varepsilon
\end{aligned}
$$

o que mostra que $y(\cdot)$ é contínua para todo $t \in\left[t_{0}, t_{0}+\alpha\right]$. Logo, $y(t)$ é uma solução contínua da equação (2.6) e, assim, pela Proposição 1 é uma solução do P.V.I. (2.4).

\section{A unicidade de solução}

Para finalizar a demonstração do Teorema 1, mostraremos, nesta etapa, que a solução encontrada na etapa 3 é única no intervalo $\left[t_{0}, t_{0}+\alpha\right]$. Para isso, provaremos, inicialmente, o seguinte resultado.

Lema 2. Seja $w:\left[t_{0}, c\right) \rightarrow \mathbb{R}, c>t_{0}$, uma função contínua e não negativa. Se

$$
w(t) \leqslant K \int_{t_{0}}^{t} w(s) d s, \quad t_{0} \leqslant t<c
$$

onde $K>0$, então $w(t)=0$ para todo $t_{0} \leqslant t<c$. 
Demonstração. Dado $t \in\left[t_{0}, c\right)$ e $\varepsilon>0$ defina

$$
U(t)=\varepsilon+\int_{t_{0}}^{t} w(s) d s
$$

Então, como $w$ é contínua, temos que $U^{\prime}(t)=w(t)$ e, por hipótese,

$$
\begin{aligned}
w(t) & \leqslant K \int_{t_{0}}^{t} w(s) d s \\
& \leqslant K\left(\varepsilon+\int_{t_{0}}^{t} w(s) d s\right) \\
& =K U(t) .
\end{aligned}
$$

Ou seja, $U^{\prime}(t) \leqslant K U(t)$, o que implica que

$$
\frac{U^{\prime}(t)}{U(t)} \leqslant K
$$

e, portanto, que

$$
\frac{d}{d t}(\ln U(t)) \leqslant K
$$

Logo, integrando de $t_{0}$ a $t$ temos que

$$
\ln U(t)-\ln U\left(t_{0}\right) \leqslant K\left(t-t_{0}\right) .
$$

Ou seja,

$$
\begin{aligned}
& \ln \left(\frac{U(t)}{U\left(t_{0}\right)}\right) \leqslant K\left(t-t_{0}\right) \\
\Rightarrow & \frac{U(t)}{U\left(t_{0}\right)} \leqslant e^{K\left(t-t_{0}\right)} \\
\Rightarrow & e^{-K\left(t-t_{0}\right)} U(t) \leqslant U\left(t_{0}\right)=\varepsilon .
\end{aligned}
$$

Como $\varepsilon>0$ é arbitrário, fazendo $\varepsilon$ se aproximar de zero, concluímos que

$$
e^{-K\left(t-t_{0}\right)} U(t) \leqslant 0,
$$

o que implica que $U(t) \leqslant 0$, pois $e^{-K\left(t-t_{0}\right)}>0$. Por outro lado, é claro que $U(t) \geqslant 0$. Logo, $U(t)=0$. Assim, concluímos que $0 \leqslant w(t) \leqslant K U(t)=0$ para todo $t_{0} \leqslant t<c$, e, portanto, que $w(t)=0$ para todo $t_{0} \leqslant t<c$.

Proposição 4. A solução do P.V.I. (2.4) encontrada na Proposição 3 é única.

Demonstração. Suponha que $z(t)$ seja outra solução contínua de (2.4). Então, temos que

$$
y(t)=y_{0}+\int_{t_{0}}^{t} f(s, y(s)) d s \quad \text { e } \quad z(t)=y_{0}+\int_{t_{0}}^{t} f(s, z(s)) d s
$$


Logo,

$$
\begin{aligned}
|y(t)-z(t)| & =\left|\int_{t_{0}}^{t} f(s, y(s)) d s-\int_{t_{0}}^{t} f(s, z(s)) d s\right| \\
& \leqslant \int_{t_{0}}^{t}|f(s, y(s))-f(s, z(s))| d s \\
& \leqslant L \int_{t_{0}}^{t}|y(s)-z(s)| d s .
\end{aligned}
$$

Fazendo agora $|y(t)-z(t)|=g(t)$ temos que $g$ é contínua e não negativa em $\left[t_{0}, t_{0}+\alpha\right] \mathrm{e}$ $g(t) \leqslant L \int_{t_{0}}^{t} g(s) d s$. Logo, pelo Lema 2 , segue que $g(t)=0$ para todo $t \in\left[t_{0}, t_{0}+\alpha\right]$ e, assim, $y(t)-z(t)=0$ para todo $t \in\left[t_{0}, t_{0}+\alpha\right]$. Ou seja, $y(t)=z(t)$ para todo $t \in\left[t_{0}, t_{0}+\alpha\right]$. Portanto, concluímos a unicidade da solução.

As quatro etapas apresentadas acima mostram que nas condições do Teorema 1 existe uma única solução $y(\cdot)$ de $(2.4)$ em $\left[t_{0}, t_{0}+\alpha\right]$. A seguir apresentaremos dois exemplos simples envolvendo o Teorema 1.

Exemplo 4. Vejamos que o P.V.I.

$$
\left\{\begin{array}{l}
y^{\prime}=1+y+y^{2} \cos (t), \\
y(0)=0
\end{array}\right.
$$

possui uma única solução em um intervalo específico.

Primeiramente, note que $f(t, y)=1+y+y^{2} \cos (t)$ e $\frac{\partial f}{\partial y}=1+2 y \cos (t)$ são contínuas em qualquer retângulo $R=\{(t, y)|0 \leqslant t \leqslant a| y \mid, \leqslant b\}$, em que $a$ e $b \in \mathbb{R}^{+}$. Assim, calculamos

$$
M=\max _{(t, y) \in R}|f(t, y)|=\max \left\{\left|1+y+y^{2} \cos (t)\right| / 0 \leqslant t \leqslant a,|y| \leqslant b\right\}=b^{2}+b+1 .
$$

Desse modo, $\alpha=\min \left\{a, \frac{b}{b^{2}+b+1}\right\}$. Como podemos tomar qualquer valor para $a$, temos que o maior valor de $\alpha$ será quando $\frac{b}{b^{2}+b+1}$ for máximo (tomamos o maior valor de $\alpha$ a fim de obter o maior intervalo em que garantimos a existência e unicidade local).

É fácil ver que o máximo de $\frac{b}{b^{2}+b+1}$ é $\frac{1}{3}$. Assim sendo, o maior valor de $\alpha$ é $\frac{1}{3}$ e, pelo Teorema 1, temos que o P.V.I.

$$
\left\{\begin{array}{l}
y^{\prime}(t)=1+y+y^{2} \cos (t) \\
y(0)=0
\end{array}\right.
$$

possui uma única solução no intervalo $0 \leqslant t \leqslant \frac{1}{3}$.

Exemplo 5. Vejamos agora o seguinte P.V.I.

$$
\left\{\begin{array}{l}
y y^{\prime}-4 t=0 \\
y(0)=0
\end{array}\right.
$$


Temos que $f(t, y)=\frac{4 t}{y}$ e $\frac{\partial f}{\partial y}=-\frac{4 t}{y^{2}}$. Note que, embora ambas sejam contínuas em algumas regiões, nenhuma delas é contínua em algum retângulo da forma $R=\{(t, y)|0 \leqslant t \leqslant a| y \mid, \leqslant b\}$, pois não estão definidas no ponto $(0,0)$. Logo, não podemos garantir a unicidade das soluções para esse P.V.I.

Note que, neste caso, temos a existência de solução, mas não temos a unicidade. De fato, temos, por exemplo, que tanto $y(t)=2 t$ quanto $z(t)=-2 t$ são soluções para o P.V.I.

$$
\left\{\begin{array}{l}
y y^{\prime}-4 t=0 \\
y(0)=0
\end{array}\right.
$$

pois considerando, primeiramente, $y(t)=2 t$ temos que $\frac{d y}{d t}=2 \mathrm{e}$, assim, substituindo na equação, obtemos que

$$
2 t \cdot 2-4 t=4 t-4 t=0
$$

e $y(0)=0$. Já considerando $z(t)=-2 t$ temos que $\frac{d z}{d t}=-2$ e, então, ao substituirmos na equação, temos

$$
(-2 t) \cdot(-2)-4 t=4 t-4 t=0,
$$

$\mathrm{e} z(0)=0$. 



\section{3}

\section{EQUAÇÕES DIFERENCIAIS ORDINÁRIAS DE PRIMEIRA ORDEM}

Neste capítulo, nosso objetivo é apresentar alguns métodos de resolução para equações diferenciais ordinárias de primeira ordem que aparecem de maneira mais frequente. Observamos que o Teorema de Existência e Unicidade apresentado no capítulo anterior garante a existência de soluções dos problemas que serão apresentados.

\subsection{Equações Diferenciais Lineares}

Iniciaremos nosso estudo com uma classe de EDOs de primeira ordem que são mais simples e que aparecem com frequência em aplicações, as equações diferenciais lineares.

Definição 9. Uma equação diferencial linear de primeira ordem é uma equação da forma

$$
y^{\prime}+a(t) y=b(t)
$$

onde $a(t)$ e $b(t)$ são funções contínuas num intervalo $I$.

Observação 3. Note que se $a_{0}(t), a_{1}(t)$ e $b_{1}(t)$ são funções contínuas num intervalo $I$, com $a_{1}(t) \neq 0$ em $I$, então a equação

$$
a_{1}(t) y^{\prime}+a_{0}(t) y=b_{1}(t)
$$

pode ser reescrita na forma $y^{\prime}+a(t) y=b(t)$, onde $a(t)$ e $b(t)$ são contínuas em $I$.

Observação 4. A equação (3.1) é chamada linear, pois a parte que depende de y é linear em y. De fato, definindo $g(t, y):=a(t) y$, temos que

$$
\begin{aligned}
g\left(t, \alpha_{1} y_{1}+\alpha_{2} y_{2}\right) & =a(t)\left[\alpha_{1} y_{1}+\alpha_{2} y_{2}\right] \\
& =\alpha_{1} a(t) y_{1}+\alpha_{2} a(t) y_{2} \\
& =\alpha_{1} g\left(t, y_{1}\right)+\alpha_{2} g\left(t, y_{2}\right) .
\end{aligned}
$$


Exemplo 6. A equação $y^{\prime}=t^{3} \operatorname{sen}(t)-y \cos (t)$ é uma EDO linear de $1^{\mathrm{a}}$ ordem. De fato, note que, neste caso, $g(t, y)=y \cos (t) \mathrm{e}$

$$
\begin{aligned}
g\left(t, \alpha_{1} y_{1}+\alpha_{2} y_{2}\right) & =\left(\alpha_{1} y_{1}+\alpha_{2} y_{2}\right) \cos (t) \\
& =\alpha_{1} y_{1} \cos (t)+\alpha_{2} y_{2} \cos (t) \\
& =\alpha_{1} g\left(t, y_{1}\right)+\alpha_{2} g\left(t, y_{2}\right)
\end{aligned}
$$

Exemplo 7. A equação $y^{\prime}=t y^{2}+\operatorname{sen}(t)$ não é uma EDO linear de $1^{\mathrm{a}}$ ordem. Neste caso, $g(t, y)=t y^{2} \mathrm{e}$

$$
\begin{aligned}
g\left(t, \alpha_{1} y_{1}+\alpha_{2} y_{2}\right) & =\left(\alpha_{1} y_{1}+\alpha_{2} y_{2}\right)^{2} t \\
& =\left(\alpha_{1} y_{1}\right)^{2} t+2\left(\alpha_{1} y_{1}\right)\left(\alpha_{2} y_{2}\right) t+\left(\alpha_{2} y_{2}\right)^{2} t \\
& =\alpha_{1}^{2} g\left(t, y_{1}\right)+\alpha_{2}^{2} g\left(t, y_{2}\right)+2 \alpha_{1} y_{1} \alpha_{2} y_{2} t \\
& \neq \alpha_{1} g\left(t, y_{1}\right)+\alpha_{2} g\left(t, y_{2}\right) .
\end{aligned}
$$

Observação 5. Considere o P.V.I. associado à equação (3.1)

$$
\left\{\begin{array}{l}
y^{\prime}+a(t) y=b(t), \\
y\left(t_{0}\right)=y_{0}
\end{array}\right.
$$

onde $a(t)$ e $b(t)$ são contínuas num intervalo $I$ e $t_{0} \in I$. Então, pelo Teorema de Existência e Unicidade, este P.V.I. possui uma única solução local, pois as funções $f(t, y)=-a(t) y+b(t) \mathrm{e}$ $\frac{\partial f}{\partial y}(t, y)=-a(t)$ são contínuas tanto em $t$ quanto em $y$.

No que segue tentaremos encontrar uma solução para a equação (3.1) e, assim, para um P.V.I. associado a esta equação.

\subsubsection{Equações Homogêneas}

Como não é imediato encontrar uma solução para equação (3.1), inicialmente, iremos simplificá-la, colocando $b(t) \equiv 0$, e assim teremos

$$
y^{\prime}+a(t) y=0
$$

Definição 10. A equação (3.3) é chamada equação diferencial linear homogênea de primeira ordem [L.H.] associada a (3.1). Já a equação (3.1) é chamada equação diferencial linear não homogênea de primeira ordem [L.N.H.].

Supondo que $y(t) \neq 0$ em $I$, a equação (3.3) pode facilmente ser resolvida. De fato, isolando $y^{\prime}$ e dividindo ambos os membros por $y$, obtemos

$$
\frac{y^{\prime}}{y}=-a(t) \text {. }
$$


Como $\frac{y^{\prime}}{y}=\frac{d}{d t}(\ln |y(t)|)$, podemos reescrever a equação acima como

$$
\frac{d}{d t}(\ln |y(t)|)=-a(t)
$$

e agora, integrando ambos os membros desta equação, ficamos com

$$
\ln |y(t)|=-\int a(t) d t+c_{1}
$$

onde $c_{1}$ é uma constante de integração. Por meio da exponencial, obtemos

$$
|y(t)|=e^{\left(-\int a(t) d t+c_{1}\right)}
$$

o que implica que

$$
y(t)=c e^{-\int a(t) d t}
$$

Assim, vemos que $y(t)$ dada por (3.4) é uma solução de (3.3) e, além disso, qualquer outra solução será desta forma para algum $c \in \mathbb{R}$. Assim, podemos considerar a seguinte definição.

Definição 11. Dizemos que (3.4) é a solução geral da equação diferencial linear homogênea [L.H.].

Exemplo 8. Encontremos a solução do P.V.I.

$$
\left\{\begin{array}{l}
y^{\prime}+2 y=0 \\
y(0)=1
\end{array}\right.
$$

Neste caso, $a(t)=2 \mathrm{e}$, assim, temos que a solução geral é dada por

$$
y(t)=c e^{-\int 2 d t}=c e^{-2 t} .
$$

Agora, como $y(0)=1$, temos que

$$
1=y(0)=c e^{0} \Rightarrow c=1 .
$$

Desse modo, a solução do P.V.I. é dada por

$$
y(t)=e^{-2 t}
$$

Proposição 5. Sejam $y_{1}$ e $y_{2}$ soluções da [L.H.]. Então:

(i) $y_{1}+y_{2}$ também é solução da [L.H.];

(ii) $c y_{1}, c \in \mathbb{R}$, também é solução da [L.H.];

(iii) a função $y(t) \equiv 0$ é solução da [L.H.]. 
Demonstração. (i) Substituindo $y_{1}+y_{2}$ em [L.H.] temos que

$$
\begin{aligned}
\left(y_{1}+y_{2}\right)^{\prime}+a(t)\left(y_{1}+y_{2}\right) & =y_{1}^{\prime}+y_{2}^{\prime}+a(t) y_{1}+a(t) y_{2} \\
& =\left(y_{1}^{\prime}+a(t) y_{1}\right)+\left(y_{2}^{\prime}+a(t) y_{2}\right) \\
& =0
\end{aligned}
$$

pois $y_{1}$ e $y_{2}$ são soluções de [L.H.]. Logo, $y_{1}+y_{2}$ é solução da [L.H.].

(ii) Substituindo $c y_{1}$ em [L.H.] temos

$$
\left(c y_{1}\right)^{\prime}+a(t)\left(c y_{1}\right)=c y_{1}^{\prime}+c a(t) y_{1}=c\left(y_{1}^{\prime}+a(t) y_{1}\right)=0
$$

pois $y_{1}$ é solução de [L.H.]. Logo, $c y_{1}$ é solução da [L.H.].

(iii) Sendo $y(t) \equiv 0$ então $y^{\prime}(t) \equiv 0$ e substituindo em [L.H.] temos

$$
y^{\prime}+a(t) y=0+a(t) 0=0
$$

Portanto, $y(t) \equiv 0$ é solução da [L.H.].

Observação 6. A proposição anterior nos permite dizer que o conjunto das soluções da [L.H.] é um espaço vetorial de dimensão 1 e que $y(t)=e^{-\int a(t) d t}$ é uma base para este espaço.

\subsubsection{Equações Não Homogêneas}

Consideremos agora a equação não homogênea

$$
y^{\prime}+a(t) y=b(t)
$$

e tentemos encontrar uma solução para ela como fizemos para a [L.H.]. Se pudéssemos fazer algo para que a [L.N.H.] possa ser reescrita como

$$
\frac{d}{d t}\left({ }^{\prime \prime} \operatorname{alg} o^{\prime \prime}\right)=b(t)
$$

nosso problema estaria resolvido, pois bastaria integrar ambos os membros para encontrarmos o valor de "algo". Mas $y^{\prime}+a(t) y$ não parece ser a derivada de uma expressão simples. Procuraremos, então, uma função $\mu(t)$, contínua e diferenciável, tal que multiplicando ambos os membros da [L.N.H.] por $\mu(t)$ e considerando a equação equivalente

$$
\mu(t) y^{\prime}+\mu(t) a(t) y=\mu(t) b(t)
$$

a expressão $\mu(t) y^{\prime}+\mu(t) a(t) y$ seja a derivada de alguma sentença simples.

Agora, observe que

$$
\frac{d}{d t}(\mu(t) y)=\mu(t) y^{\prime}+\mu^{\prime}(t) y
$$


Logo,

$$
\begin{aligned}
\mu(t) y^{\prime}+a(t) \mu(t) y=\frac{d}{d t}(\mu(t) y) & \Leftrightarrow \mu(t) y^{\prime}+a(t) \mu(t) y=\mu(t) y^{\prime}+\mu^{\prime}(t) y \\
& \Leftrightarrow \mu^{\prime}(t)=a(t) \mu(t) \\
& \Leftrightarrow \mu^{\prime}(t)-a(t) \mu(t)=0 .
\end{aligned}
$$

Mas, note que, $\mu^{\prime}(t)-a(t) \mu(t)=0$ é uma EDO linear de primeira ordem homogênea, para a qual já temos um método de resolução apresentado na Seção 3.1.1. Assim, resolvendo esta equação temos que

$$
\mu(t)=c e^{\int a(t) d t} .
$$

Como precisamos de apenas uma função $\mu(t)$ satisfazendo as condições acima, podemos tomar $c=1$ e, então, considerar a função

$$
\mu(t)=e^{\int a(t) d t}
$$

Desse modo, a equação (3.5) pode ser escrita como

$$
\frac{d}{d t}(\mu(t) y)=\mu(t) b(t)
$$

e, integrando ambos os membros, obtemos

$$
\mu(t) y=\int \mu(t) b(t) d t+c
$$

o que equivale a

$$
y(t)=\frac{1}{\mu(t)}\left(\int \mu(t) b(t) d t+c\right)=e^{-\int a(t) d t}\left(c+\int e^{\int a(t) d t} b(t) d t\right) .
$$

Ou seja,

$$
y(t)=c e^{-\int a(t) d t}+e^{-\int a(t) d t} \int e^{\int a(t) d t} b(t) d t
$$

é a solução geral da equação linear não homogênea.

Observação 7. Note que a $1^{\mathrm{a}}$ parcela da solução geral de [L.N.H.] é a solução geral da equação linear homogênea associada e que a $2^{\mathrm{a}}$ parcela é uma solução particular da equação não homogênea, obtida quando $c=0$, ou seja,

$$
y_{p}(t)=0 e^{-\int a(t) d t}+e^{-\int a(t) d t} \int e^{\int a(t) d t} b(t) d t=e^{-\int a(t) d t} \int e^{\int a(t) d t} b(t) d t .
$$

Assim, a solução geral da [L.N.H.] é a soma da solução geral da [L.H.] associada com uma solução particular da [L.N.H.]. Esta observação será útil quando tratarmos das EDOs de segunda ordem.

Definição 12. A função $\mu(t)=e^{\int a(t) d t}$ é chamada fator integrante para a equação linear não homogênea. 
Observação 8. Note que para uma EDO linear não homogênea é mais simples repetir o processo apresentado acima do que decorar a fórmula (3.6).

Exemplo 9. Encontremos a solução do P.V.I.

$$
\left\{\begin{array}{l}
y^{\prime}-\frac{y}{t}=t e^{t}, \quad t \geqslant 1, \\
y(1)=e-1
\end{array}\right.
$$

Neste caso, $a(t)=-\frac{1}{t}$. Então,

$$
\mu(t)=e^{\int a(t) d t}=e^{\int-\frac{1}{t} d t}=e^{-\ln |t|}=\frac{1}{e^{\ln |t|}}=\frac{1}{t} .
$$

Agora, multiplicando toda a equação por $\mu(t)$ temos

$$
\frac{y^{\prime}}{t}-\frac{y}{t^{2}}=e^{t}
$$

que equivale a

$$
\frac{d}{d t}\left(\frac{y}{t}\right)=e^{t}
$$

Integrando ambos os membros da equação acima obtemos

$$
\frac{y}{t}=\int e^{t} d t \Rightarrow \frac{y}{t}=e^{t}+c
$$

Logo,

$$
y(t)=t e^{t}+t c
$$

Finalmente, como

$$
y(1)=1 e^{1}+1 c \Rightarrow e-1=e+c \Rightarrow c=-1,
$$

temos que a solução do P.V.I. é dada por

$$
y(t)=t e^{t}-t
$$

\subsubsection{Algumas Aplicações}

\subsubsection{Modelo Populacional de Malthus}

Como vimos anteriormente, a taxa de variação populacional segundo o modelo de Malthus é dada pela EDO linear homogênea de primeira ordem $p^{\prime}-k p=0$, onde $a(t)=-k$ e, assim, tem como solução

$$
p(t)=c e^{-\int-k d t}=c e^{k t} .
$$

Sendo a condição inicial $p(0)=p_{0}$ temos que

$$
p_{0}=p(0)=c e^{k 0} \Rightarrow p_{0}=c .
$$

Desse modo,

$$
p(t)=p_{0} e^{k t}
$$


Note que, se a taxa de natalidade para essa população é maior que a de mortalidade, ou seja, $k>0$, então, como já esperávamos, a população cresce. E se a taxa de natalidade para essa população é menor que a de mortalidade, ou seja, $k<0$, então, também como já esperávamos, a população decresce.

Exemplo 10. Uma colônia de bactérias cresce a uma razão proporcional ao número de bactérias presente. Se o número duplica a cada 24 horas, quantas horas serão necessárias para que o número de bactérias aumente cem vezes de sua quantidade inicial?

O número de bactérias em um instante $t$ é dado por

$$
p(t)=p_{0} e^{k t}
$$

Sabemos que o número de bactérias dobra a cada 24 horas, então

$$
p(24)=p_{0} e^{k 24}=2 p_{0} \Rightarrow k \cong 0,029 \text {. }
$$

Desse modo,

$$
p(t)=p_{0} e^{0,029 t}
$$

Como desejamos saber quando o número de bactérias aumentará em cem vezes de sua quantidade inicial, basta substituir $p(t)=100 p_{0}$ e obtemos

$$
100 p_{0}=p_{0} e^{0,029 t} \Rightarrow e^{0,029 t}=100 \Rightarrow t \cong 158,8 \text {. }
$$

Desse modo, para que o número de bactérias aumente em cem vezes de sua quantidade inicial são necessárias aproximadamente 158 horas e 48 minutos.

Como já observamos anteriormente, no modelo de Malthus só são consideradas as mortes por causas naturais. Assim, ele não é um modelo adequado para todos os tipos de populações, pois quando $k<0$ e $t$ tende ao infinito temos que $p(t)$ tende a 0 e, assim, a população será extinta, e quando $k>0$ e $t$ tende ao infinito temos que $p(t)$ tende ao infinito e, assim, esta população crescerá indefinidamente. Outro modelo populacional mais adequado (de Verhulst) será trabalhado mais a frente.

\subsubsection{Decaimento Radioativo}

Como também já vimos, a taxa de desintegração de um elemento radioativo é dada pela EDO linear homogênea de primeira ordem $Q^{\prime}+K Q=0$, com $K>0$. Aqui $a(t)=K$ e, assim, a solução da EDO é dada por

$$
Q(t)=c e^{-\int K d t}=c e^{-K t} .
$$

Considerando a condição inicial $Q(0)=Q_{0}$ temos

$$
Q_{0}=Q(0)=c e^{-K 0} \Rightarrow Q_{0}=c .
$$


Assim sendo, temos que

$$
Q(t)=Q_{0} e^{-K t}
$$

Observemos que vale uma equação semelhante quando, ao invés de considerarmos a quantidade do elemento, consideramos a massa do elemento. Se $m$ denota a massa do elemento, o problema fica

$$
m^{\prime}+K m=0
$$

Se $m(0)=m_{0}$, a solução é dada por

$$
m(t)=m_{0} e^{-K t}
$$

Para o próximo exemplo observamos que a meia-vida de um elemento ou substância radioativa é definida como o tempo necessário para a decomposição da metade do elemento ou da substância.

Exemplo 11. Um químico possui 200 gramas de um elemento radioativo e sabe que ele possui meia-vida de 4 dias. Este químico deseja saber em quanto tempo terá 75 gramas do elemento.

A quantidade de elemento em qualquer instante é dada por

$$
m(t)=200 e^{-K t} .
$$

Além disso, como sabemos que a meia-vida desse elemento é de 4 dias, então

$$
Q(4)=\frac{1}{2} Q_{0}=\frac{1}{2} 200=100 \text {. }
$$

Por outro lado, por (3.7) temos que

$$
Q(4)=200 e^{-K 4}
$$

o que implica que

$$
200 e^{-4 K}=100 \Rightarrow e^{-4 K}=\frac{1}{2} \Rightarrow K \cong 0,1733 .
$$

Desse modo, a solução do nosso problema é dada por

$$
Q(t)=200 e^{-0,1733 t}
$$

Agora, como desejamos saber quando restará 75 gramas do elemento, basta substituir esse valor em $Q(t)$ na equação acima, e obtemos

$$
75=200 e^{-0,1733 t} \Rightarrow e^{-0,1733 t}=0,375 \Rightarrow t \cong 5,66 .
$$

Ou seja, teremos 75 gramas do elemento aproximadamente em 5,66 dias, ou 5 dias, 15 horas e 50 minutos.

Observação 9. É interessante notar que a desintegração radioativa é utilizada, por exemplo, na datação por carbono-14 e também para descobrir a falsificação de obras de arte, entre outras aplicações. 


\subsubsection{Diluição de Misturas}

Consideremos um problema sobre mistura de fluidos num tanque, em que $y(t)$ representa a quantidade da substância no tanque no instante $t, T_{E}(t)$ representa a taxa de entrada da substância no tanque no instante $t$ e $T_{S}(t)$ representa a taxa de saída da substância do tanque no instante $t$.

Das considerações acima, a derivada de $y$ com relação a $t$ pode ser entendida como a taxa de variação da quantidade da substância no tanque com relação ao tempo. Assim, se quisermos encontrar a quantidade da substância presente no tanque num dado instante $t$ podemos resolver a seguinte EDO

$$
y^{\prime}=T_{E}(t)-T_{S}(t),
$$

onde $T_{E}(t)$ e $T_{S}(t)$ podem depender de $y(t)$. Mais ainda, a concentração da substância presente no tanque no instante $t$ é dada por $C(t)=\frac{y(t)}{k}$, onde $k$ é a capacidade total do tanque.

Exemplo 12. Um tanque contém 100 litros de água salgada. É adicionado, neste tanque, água salgada à razão de 5 litros por minuto, com uma concentração de sal de $2 \mathrm{~g} / \ell$. Ao mesmo tempo, a mistura deixa o tanque através de um buraco à mesma razão. A mistura do tanque é continuamente agitada, de modo a manter a solução homogênea, ou seja, a concentração é a mesma em todo tanque. Se, inicialmente, a mistura contém uma concentração de $1 \mathrm{~g} / \ell$, determine a concentração num instante futuro.

Note primeiramente que, como a água que entra tem concentração de sal de $2 \mathrm{~g} / \ell$ e está entrando numa razão de $5 \ell / \mathrm{min}$, então a quantidade de sal adicionado ao tanque corresponde a $2 \cdot 5 \mathrm{~g} / \mathrm{min}=10 \mathrm{~g} / \mathrm{min}$. Da mesma forma, como a água deixa o tanque com uma concentração de sal de $\frac{y(t)}{100} g / \ell$, numa razão de $5 \ell / \min$, temos que a quantidade de sal deixando o tanque corresponde a $5 \cdot \frac{y(t)}{100} \mathrm{~g} / \mathrm{min}=\frac{y(t)}{20} \mathrm{~g} / \mathrm{min}$. Logo, temos que $T_{E}(t)=10$ e $T_{S}(t)=\frac{y(t)}{20}$ e a variação da quantidade de sal no tanque é dada por

$$
y^{\prime}(t)=10-\frac{y(t)}{20}
$$

ou seja,

$$
y^{\prime}(t)+\frac{y(t)}{20}=10
$$

que é uma EDO linear não homogênea de primeira ordem. Então, para resolver esta EDO encontremos, inicialmente, o fator integrante. Como aqui $a(t)=\frac{1}{20}$, temos que

$$
\mu(t)=e^{\int a(t) d t}=e^{\int \frac{1}{20} d t}=e^{\frac{t}{20}} .
$$

Multiplicando a EDO por $\mu(t)$ ficamos com

$$
e^{\frac{t}{20}} y^{\prime}(t)+e^{\frac{t}{20}} \frac{y(t)}{20}=10 e^{\frac{t}{20}}
$$

ou seja,

$$
\frac{d}{d t}\left(e^{\frac{t}{20}} y(t)\right)=10 e^{\frac{t}{20}}
$$


Integrando ambos os membros da igualdade acima de $t_{0}=0$ a $t$ obtemos

$$
e^{\frac{t}{20}} y(t)-y(0)=200 e^{\frac{t}{20}}-200
$$

Como $y(0)=100$, concluímos que

$$
y(t)=200-100 e^{\frac{-t}{20}}
$$

Logo, a concentração de sal no tanque no instante $t$ será de

$$
C(t)=\frac{y(t)}{100}=2-e^{\frac{-t}{20}}
$$

Observação 10. Note que no exemplo acima

$$
\lim _{t \rightarrow \infty} C(t)=\lim _{t \rightarrow \infty}\left(2-e^{\frac{-t}{20}}\right)=2 .
$$

Ou seja, depois de muito tempo, a concentração de sal no tanque tende a ser de $2 \mathrm{~g} / \ell$.

Observação 11. Problemas que envolvem a quantidade de um certo elemento presente em um recipiente, geralmente, podem ser descritos da mesma forma que no Exemplo 12 como, por exemplo, a concentração de oxigênio em uma sala com ventilação controlada.

\subsubsection{Variação da Temperatura de um Corpo}

Como já foi visto, a variação da temperatura de um corpo pode ser descrita pela EDO

$$
T^{\prime}(t)+K T(t)=K T_{a}(t)
$$

ou seja, a variação da temperatura do corpo é proporcional à diferença entre as temperaturas do corpo e do meio ambiente. A seguir consideramos o caso em que a temperatura $T_{a}$ do ambiente é constante e o caso em que esta temperatura varia com o tempo.

\section{$1^{\circ}$ Caso: Temperatura do Ambiente Constante}

Se a temperatura do ambiente é constante e não nula, ou seja, $T_{a}(t)=T_{a} \neq 0$ para todo $t$, então a EDO

$$
T^{\prime}(t)+K T(t)=K T_{a},
$$

é uma EDO linear não homogênea de primeira ordem e, assim, sabemos encontrar sua solução. Como, neste caso, $a(t)=K$, então o fator integrante é dado por

$$
\mu(t)=e^{\int K d t}=e^{K t} .
$$

Muliplicando a EDO por $\mu(t)$ temos

$$
e^{K t} T^{\prime}(t)+e^{K t} K T(t)=e^{K t} K T_{a}
$$


ou seja,

$$
\frac{d}{d t}\left(e^{K t} T(t)\right)=e^{K t} K T_{a}
$$

Integrando ambos os membros da igualdade acima obtemos

$$
\begin{aligned}
e^{K t} T(t) & =\int e^{K t} K T_{a} d t \\
& =T_{a} \int e^{K t} K d t \\
& =T_{a}\left(e^{K t}+c_{1}\right) \\
& =T_{a} e^{K t}+c .
\end{aligned}
$$

Logo,

$$
T(t)=c e^{-K t}+T_{a} .
$$

Supondo que a temperatura inicial é dada por $T(0)=T_{0}$, temos que

$$
T_{0}=T(0)=c e^{-K 0}+T_{a} \Rightarrow c=T_{0}-T_{a},
$$

ou seja, a temperatura do corpo, conhecida sua temperatura inicial, é dada por

$$
T(t)=\left(T_{0}-T_{a}\right) e^{-K t}+T_{a}
$$

Observação 12. É interessante notar os seguintes fatos:

i) Quando $T_{0}>T_{a}$, temos que

$$
T_{0}-T(t)=T_{0}-\left[\left(T_{0}-T_{a}\right) e^{-K t}+T_{a}\right]=\left(T_{0}-T_{a}\right)\left(1-e^{-K t}\right)>0,
$$

ou seja, $T(t)<T_{0}$. Assim, como já esperávamos, a temperatura do corpo deve diminuir com o passar do tempo.

ii) Quando $T_{0}<T_{a}$, temos que

$$
T_{0}-T(t)=T_{0}-\left[\left(T_{0}-T_{a}\right) e^{-K t}+T_{a}\right]=\left(T_{0}-T_{a}\right)\left(1-e^{-K t}\right)<0,
$$

ou seja, $T(t)>T_{0}$. Novamente, como esperávamos, a temperatura do corpo deve aumentar com o passar do tempo.

iii) Finalmente, quando $T_{0}=T_{a}$, temos que

$$
T_{0}-T(t)=T_{0}-\left[\left(T_{0}-T_{a}\right) e^{-K t}+T_{a}\right]=0,
$$

ou seja, $T(t)=T_{0}$. Ou seja, $T(t)$ permanece constante.

Observação 13. Note que, quando $t$ tende ao infinito, $T(t)$ tende a $T_{a}$. De fato,

$$
\lim _{t \rightarrow \infty} T(t)=\lim _{t \rightarrow \infty}\left[\left(T_{0}-T_{a}\right) e^{-K t}+T_{a}\right]=0+T_{a}=T_{a} .
$$

Por este motivo, $T_{a}$ é chamada de temperatura de equilíbrio. 


\section{$2^{\circ}$ Caso: Temperatura do Ambiente não Constante}

Suponhamos agora que a temperatura do ambiente varia com o tempo ao receber ou ceder calor ao corpo. Sejam $m$ e $m_{a}$ as massas do corpo e do meio ambiente, respectivamente, e $c$ e $c_{a}$ os calores específicos do corpo e do meio ambiente, respectivamente. Supondo que não haja mudanças de estado físico, sabe-se que a lei de conservação da quantidade de calor é descrita por

$$
m c\left(T_{0}-T\right)=m_{a} c_{a}\left(T_{a}-T_{a, 0}\right),
$$

onde $T=T(t)$ e $T_{a}=T_{a}(t)$ são as temperaturas do corpo e do ambiente, respectivamente, no instante $t$, e $T_{0}=T(0)$ e $T_{a, 0}=T_{a}(0)$. Assim, temos que a temperatura do ambiente varia da seguinte forma

$$
T_{a}=\frac{m c}{m_{a} c_{a}}\left(T_{0}-T\right)+T_{a, 0}
$$

Substituindo na equação $T^{\prime}+K T=K T_{a}$ obtemos

$$
\begin{aligned}
T^{\prime}+K T & =K\left(\frac{m c}{m_{a} c_{a}}\left(T_{0}-T\right)+T_{a, 0}\right) \\
& =K\left(\frac{m c}{m_{a} c_{a}}\right)\left(T_{0}-T\right)+K T_{a, 0} .
\end{aligned}
$$

Logo, temos a seguinte EDO

$$
T^{\prime}+K\left(1+\frac{m c}{m_{a} c_{a}}\right) T=K\left(T_{a, 0}+\frac{m c}{m_{a} c_{a}} T_{0}\right)
$$

Como $m, c, m_{a}$ e $c_{a}$ são constantes, podemos tomar $A=\frac{m c}{m_{a} c_{a}}$ e a EDO acima fica

$$
T^{\prime}+K(1+A) T=K\left(T_{a, 0}+A T_{0}\right),
$$

que é uma EDO linear não homogênea de primeira ordem, com $a(t)=K(1+A)$. Procedendo como no caso anterior, vemos que sua solução satisfazendo a condição inicial $T(0)=T_{0}$ é dada por

$$
T(t)=\frac{T_{0}-T_{a, 0}}{1+A} e^{-K(1+A) t}+\frac{T_{a, 0}+A T_{0}}{1+A} .
$$

Observação 14. Novamente, é fácil verificar os seguintes fatos:

i) $T_{0}>T_{a, 0} \Rightarrow T(t)$ decresce com o tempo e, por consequência, $T_{a}(t)$ cresce.

ii) $T_{0}<T_{a, 0} \Rightarrow T(t)$ cresce com o tempo e, por consequência, $T_{a}(t)$ decresce.

iii) $T_{0}=T_{a, 0} \Rightarrow T(t)$ e $T_{a}(t)$ são constantes iguais a $T_{0}$.

Observação 15. Note que

$$
\lim _{t \rightarrow \infty} T(t)=\lim _{t \rightarrow \infty}\left(\frac{T_{0}-T_{a, 0}}{1+A} e^{-k(1+A) t}+\frac{T_{a, 0}+A T_{0}}{1+A}\right)=\frac{T_{a, 0}+A T_{0}}{1+A} .
$$


Utilizando o que foi visto anteriormente, sabemos que $T_{a}(t)=A\left(T_{0}-T(t)\right)+T_{a, 0}$. Assim,

$$
\lim _{t \rightarrow \infty} T_{a}(t)=\lim _{t \rightarrow \infty}\left[A\left(T_{0}-T(t)\right)+T_{a, 0}\right]=A\left(T_{0}-\frac{T_{a, 0}+A T_{0}}{1+A}\right)+T_{a, 0}=\frac{T_{a, 0}+A T_{0}}{1+A} .
$$

Dessa forma, neste caso, chamaremos $\frac{T_{a, 0}+A T_{0}}{1+A}$ de temperatura de equilíbrio.

Exemplo 13. Um detetive encontra uma vítima de assassinato às 9 horas da manhã em um quarto de hotel. A temperatura do corpo é de $32,4^{\circ} \mathrm{C}$. Depois de uma hora a temperatura do corpo é de $31,7^{\circ} \mathrm{C}$. Sabendo que durante todo o tempo o ar-condicionado esteve ligado mantendo a temperatura do quarto em $20,6^{\circ} \mathrm{C}$, estimemos a hora do crime.

Sabemos que $T_{0}=T(0)=32,4^{\circ} C, T(1)=31,7^{\circ} C$, e que a temperatura do ambiente é constante igual a $T_{a}=20,6^{\circ} \mathrm{C}$. Logo, a temperatura do corpo em um instante qualquer é dada por

$$
T(t)=\left(T_{0}-T_{a}\right) e^{-K t}+T_{a}=(32,4-20,6) e^{-K t}+20,6=11,8 e^{-K t}+20,6 .
$$

Como sabemos o valor de $T(1)$, então

$$
T(1)=11,8 e^{-K 1}+20,6 \Rightarrow 31,7=11,8 e^{-K}+20,6 \Rightarrow e^{-K} \cong 0,9408 \Rightarrow K \cong 0,061 .
$$

Desse modo, podemos tomar

$$
T(t)=11,8 e^{-0,061 t}+20,6
$$

Sabemos também que a temperatura de um ser humano saudável varia entre $36^{\circ} \mathrm{C}$ e $37^{\circ} \mathrm{C}$. Assim, substituindo esses valores na equação temos

$$
\begin{gathered}
37=11,8 e^{-0,061 t}+20,6 \Rightarrow t \cong-5,4 ; \\
36=11,8 e^{-0,061 t}+20,6 \Rightarrow t \cong-4,365 .
\end{gathered}
$$

Note que já era esperado encontrar um tempo negativo, pois o assassinato ocorreu antes da chegada do detetive, então, o corpo já havia cedido calor. Agora, $t=-5,4$ equivale à 5 horas e 24 minutos antes das 9 horas, ou seja, às 3 horas e 36 minutos da manhã, e que $t=-4,365$ equivale à 4 horas e 22 minutos antes das 9 horas, ou seja, às 4 horas e 38 minutos da manhã. Então, o crime ocorreu entre as $3: 36$ e $4: 38$.

\subsubsection{A Equação de Bernoulli}

Uma equação da forma

$$
y^{\prime}+p(t) y=q(t) y^{n}
$$

onde $p(t)$ e $q(t)$ são funções contínuas em algum intervalo $I$ da reta e $n \in \mathbb{R}$, é chamada Equação de Bernoulli. Observe que, quando $n=0$ ou $n=1$, a Equação de Bernoulli é uma equação linear e pode ser resolvida como visto anteriormente. Entretanto, se $n \neq 0$ e $n \neq 1$ esta equação 
não é linear, mas pode ser transformada em uma equação linear fazendo a mudança de variável $z=y^{1-n}$. De fato,

$$
z=y^{1-n} \Longleftrightarrow y=z^{\frac{1}{1-n}} \Rightarrow y^{\prime}=\frac{z^{\prime}}{1-n} z^{\frac{n}{1-n}}
$$

Substituindo na Equação de Bernoulli temos que

$$
\frac{z^{\prime}}{1-n} z^{\frac{n}{1-n}}+p(t) z^{\frac{1}{1-n}}=q(t) z^{\frac{n}{1-n}}
$$

Logo, multiplicando por $(1-n)$ encontramos

$$
z^{\prime} z^{\frac{n}{1-n}}+(1-n) p(t) z^{\frac{1}{1-n}}=(1-n) q(t) z^{\frac{n}{1-n}}
$$

Notando que $z \cdot z^{\frac{n}{1-n}}=z^{\frac{1}{1-n}}$ e substituindo na igualdade acima temos

$$
z^{\prime} z^{\frac{n}{1-n}}+(1-n) p(t) z z^{\frac{n}{1-n}}=(1-n) q(t) z^{\frac{n}{1-n}},
$$

que é equivalente a

$$
z^{\prime}+(1-n) p(t) z=(1-n) q(t)
$$

Veja que esta nova EDO é linear não homogênea de primeira ordem e pode ser resolvida pelo método do fator integrante visto na Seção 3.1.2. Assim, podemos encontrar uma solução para a equação de Bernoulli.

Exemplo 14. Encontremos a solução da equação $y^{\prime}+t^{2} y=t^{2} y^{4}$.

Neste exemplo, temos que $n=4, p(t)=t^{2}, q(t)=t^{2}$ e $z=y^{-3}$. Substituindo na equação (3.8) obtemos

$$
z^{\prime}-3 t^{2} z=-3 t^{2}
$$

e, nesta EDO, $a(t)=-3 t^{2}$ e o fator integrante é dado por

$$
\mu(t)=e^{-\int 3 t^{2} d t}=e^{-t^{3}}
$$

Multiplicando a equação por $\mu(t)$ obtemos

$$
e^{-t^{3}} z^{\prime}-e^{-t^{3}} 3 t^{2} z=-e^{-t^{3}} 3 t^{2},
$$

que é equivalente a

$$
\frac{d}{d t}\left(e^{-t^{3}} z\right)=-e^{-t^{3}} 3 t^{2}
$$

Integrando ambos os lados obtemos

$$
e^{-t^{3}} z=\int-e^{-t^{3}} 3 t^{2} d t=e^{-t^{3}}+c
$$

o que implica que

$$
z(t)=1+c e^{t^{3}}
$$

Como $y=z^{-\frac{1}{3}}$, a solução da equação dada é

$$
y(t)=\left(1+c e^{t^{3}}\right)^{-\frac{1}{3}} .
$$




\subsubsection{A Equação de Ricatti}

Uma equação da forma

$$
y^{\prime}+p(t) y+q(t) y^{2}=f(t)
$$

onde $p(t), q(t)$ e $f(t)$ são funções contínuas em algum intervalo $I$ da reta e $q(t) \neq 0$ em $I$, é chamada Equação de Ricatti. Caso seja conhecida uma solução particular $y_{1}$ da Equação de Ricatti podemos transformá-la em uma EDO linear de primeira ordem, fazendo a mudança de variável $y=y_{1}+\frac{1}{z}$. De fato, observe, primeiramente, que $y^{\prime}=y_{1}^{\prime}-\frac{z^{\prime}}{z^{2}}$. Agora, substituindo na Equação de Ricatti, obtemos

$$
\left(y_{1}^{\prime}-\frac{z^{\prime}}{z^{2}}\right)+p(t)\left(y_{1}+\frac{1}{z}\right)+q(t)\left(y_{1}+\frac{1}{z}\right)^{2}=f(t),
$$

ou seja,

$$
\left(y_{1}^{\prime}+p(t) y_{1}+q(t) y_{1}^{2}\right)-\frac{z^{\prime}}{z^{2}}+\frac{p(t)}{z}+\frac{2 q(t) y_{1}}{z}+\frac{q(t)}{z^{2}}=f(t) .
$$

Como $y_{1}$ é solução da Equação de Ricatti, a igualdade acima fica

$$
f(t)-\frac{z^{\prime}}{z^{2}}+\frac{p(t)}{z}+\frac{2 q(t) y_{1}}{z}+\frac{q(t)}{z^{2}}=f(t)
$$

o que equivale a

$$
-\frac{z^{\prime}}{z^{2}}+\frac{p(t)}{z}+\frac{2 q(t) y_{1}}{z}+\frac{q(t)}{z^{2}}=0 .
$$

Multiplicando por $z^{2}$ obtemos

$$
-z^{\prime}+p(t) z+2 q(t) y_{1} z+q(t)=0
$$

ou seja,

$$
z^{\prime}-\left[p(t)+2 q(t) y_{1}\right] z=q(t)
$$

que é uma EDO linear não homogênea de primeira ordem. Assim, conhecendo uma solução particular da Equação de Ricatti, podemos transformá-la em uma EDO linear não homogênea de primeira ordem e resolvê-la pelo método do fator integrante.

Exemplo 15. Encontremos a solução da equação $y^{\prime}+t y^{2}-2 t^{2} y+t^{3}=t+1$ conhecendo a solução $y_{1}(t)=t-1$.

Aqui temos que $p(t)=-2 t^{2}$ e $q(t)=t$. Então, substituindo na equação (3.9) obtemos

$$
z^{\prime}-\left[-2 t^{2}+2 t(t-1)\right] z=t
$$

ou seja,

$$
z^{\prime}+2 t z=t
$$

e, nesta equação, $a(t)=2 t$. Assim, temos que

$$
\mu(t)=e^{\int 2 t d t}=e^{t^{2}}
$$


Multiplicando a EDO acima por $\mu(t)$ obtemos

$$
e^{t^{2}} z^{\prime}+e^{t^{2}} 2 t z=e^{t^{2}} t
$$

que é equivalente a

$$
\frac{d}{d t}\left(e^{t^{2}} z\right)=e^{t^{2}} t
$$

Integrando ambos os membros da igualdade acima obtemos

$$
e^{t^{2}} z=\int e^{t^{2}} t d t=\frac{1}{2} \int e^{t^{2}} 2 t d t=\frac{1}{2}\left(e^{t^{2}}+c_{1}\right)=c+\frac{1}{2} e^{t^{2}} .
$$

Logo,

$$
z(t)=c e^{-t^{2}}+\frac{1}{2}
$$

Agora, como $y=y_{1}+\frac{1}{z}$, a solução da equação dada é

$$
y(t)=(t-1)+\frac{1}{c e^{-t^{2}}+\frac{1}{2}} .
$$

\subsection{Equações Diferenciais Não Lineares}

Nesta seção, estudaremos alguns tipos de equações diferenciais não lineares, com o objetivo de buscar métodos para encontrar soluções para essas equações. Observamos, primeiramente, que muitas vezes é conveniente escrever a equação

$$
y^{\prime}=f(t, y)
$$

na forma

$$
M(t, y)+N(t, y) y^{\prime}=0 .
$$

Note que isto será sempre possível, pois basta tomarmos $M(t, y)=-f(t, y)$ e $N(t, y)=1$.

\subsubsection{Equações Exatas}

Definição 13. Dada a equação diferencial de primeira ordem

$$
M(t, y)+N(t, y) \frac{d y}{d t}=0
$$

ou

$$
M(t, y) d t+N(t, y) d y=0
$$

onde $M, N: \Omega \rightarrow \mathbb{R}$ e $\Omega$ é um subconjunto aberto do $\mathbb{R}^{2}$, dizemos que ela é uma equação diferencial exata se existir uma função $V: \Omega \rightarrow \mathbb{R}$ tal que

$$
\frac{\partial V(t, y)}{\partial t}=M(t, y) \quad \text { e } \quad \frac{\partial V(t, y)}{\partial y}=N(t, y),
$$

para todo $(t, y) \in \Omega$. 
Exemplo 16. A equação $\left(t^{2}+y^{2}\right) d t+2 t y d y=0$ é exata, pois $V(t, y)=\frac{t^{3}}{3}+t y^{2}$ é tal que

$$
\frac{\partial V}{\partial t}=t^{2}+y^{2}=M(t, y) \quad \text { e } \quad \frac{\partial V}{\partial y}=2 t y=N(t, y) .
$$

Note ainda que esta não é uma equação linear.

Observação 16. Note que, se $y=y(t), t \in I$, é solução da equação (3.10) e ela é exata, então existe uma função $V(t, y)$ tal que $\frac{\partial V}{\partial t}(t, y)=M(t, y)$ e $\frac{\partial V}{\partial y}(t, y)=N(t, y)$ e, assim,

$$
\frac{\partial V}{\partial t}(t, y)+\frac{\partial V}{\partial y}(t, y) \frac{d y}{d t}=0 .
$$

Logo, se $V(t, y)$ é diferenciável, pela regra da cadeia, a equação acima é equivalente à

$$
\frac{d}{d t} V(t, y(t))=0, \quad t \in I
$$

ou seja, $V(t, y(t))=c$, para todo $t \in I$ e algum $c \in \mathbb{R}$. Logo, as soluções da equação exata são dadas implicitamente pela equação $V(t, y)=c$.

Definição 14. A função $V(t, y)$ é chamada uma integral primeira de (3.10) e as curvas definidas pela equação $V(t, y)=c$ são chamadas curvas integrais de (3.10).

Exemplo 17. No Exemplo 16, $V(t, y)=\frac{t^{3}}{3}+t y^{2}$ é uma integral primeira da equação dada e $\frac{t^{3}}{3}+t y^{2}=c$ são as curvas integrais.

Observe que no Exemplo 16 foi fácil concluir que a equação era exata e encontrar sua solução reconhecendo que o primeiro membro é a derivada de $\frac{t^{3}}{3}+t y^{2}$ em relação a $t$ e o segundo membro é a derivada desta função em relação a $y$. No entanto, para equações mais complicadas pode não ser possível fazer isto. $\mathrm{O}$ teorema a seguir nos fornece um critério para determinar se uma equação dada é exata ou não.

Teorema 2. Suponha que $M, \frac{\partial M}{\partial y}, \frac{\partial M}{\partial t}, \frac{\partial N}{\partial y}, \frac{\partial N}{\partial t}$ sejam contínuas num retângulo

$$
R=\left\{(t, y) \in \mathbb{R}^{2} \mid a<t<b \text { e } c<y<d\right\} .
$$

Então (3.10) é uma equação diferencial exata se, e somente se,

$$
\frac{\partial M}{\partial y}=\frac{\partial N}{\partial t}
$$

para todo $(t, y) \in R$.

Demonstração. Suponhamos que (3.10) seja exata. Então existe uma função $V(t, y)$ tal que

$$
\frac{\partial V}{\partial t}=M \quad \text { e } \quad \frac{\partial V}{\partial y}=N
$$


Assim,

$$
\frac{\partial M}{\partial t}=\frac{\partial^{2} V}{\partial t^{2}}, \quad \frac{\partial N}{\partial y}=\frac{\partial^{2} V}{\partial y^{2}}, \quad \frac{\partial M}{\partial y}=\frac{\partial^{2} V}{\partial y \partial t} \quad \text { e } \quad \frac{\partial N}{\partial t}=\frac{\partial^{2} V}{\partial t \partial y} .
$$

Como $\frac{\partial M}{\partial t}, \frac{\partial M}{\partial y}, \frac{\partial N}{\partial t}$ e $\frac{\partial N}{\partial y}$ são contínuas, segue que todas as derivadas parciais de $2^{\text {a }}$ ordem de $V$ são contínuas. Desse modo, pelo Teorema de Schwarz, temos que

$$
\frac{\partial^{2} V}{\partial y \partial t}=\frac{\partial^{2} V}{\partial t \partial y}
$$

ou seja,

$$
\frac{\partial M}{\partial y}=\frac{\partial N}{\partial t}
$$

Reciprocamente, suponhamos que $M$ e $N$ satisfaçam (3.11). Mostraremos que (3.10) é exata, ou seja, vamos construir uma função $V(t, y)$ satisfazendo

$$
\frac{\partial V}{\partial t}=M \quad \text { e } \quad \frac{\partial V}{\partial y}=N
$$

Note que integrando a primeira das equações acima em $t$ obtemos

$$
V(t, y)=\int M(t, y) d t+h(y)
$$

onde $h(y)$ é uma função arbitrária de $y$. Derivando esta expressão em relação a $y$, podemos concluir que

$$
\frac{\partial V}{\partial y}(t, y)=\int \frac{\partial M}{\partial y}(t, y) d t+h^{\prime}(y)
$$

Assim, $\frac{\partial V}{\partial y}(t, y)=N(t, y)$ se, e somente se,

$$
N(t, y)=\int \frac{\partial M}{\partial y}(t, y) d t+h^{\prime}(y)
$$

ou seja, se, e somente se,

$$
h^{\prime}(y)=N(t, y)-\int \frac{\partial M}{\partial y}(t, y) d t
$$

Agora, note que o segundo membro de (3.12) depende apenas de $y$. De fato,

$$
\frac{\partial}{\partial t}\left(N(t, y)-\int \frac{\partial M}{\partial y}(t, y) d t\right)=\frac{\partial N}{\partial t}(t, y)-\frac{\partial M}{\partial y}(t, y)=0,
$$

pois, por hipótese, $M$ e $N$ satisfazem (3.11). Assim, integrando (3.12) em $y$ obtemos

$$
h(y)=\int\left[N(t, y)-\int \frac{\partial M}{\partial y}(t, y) d t\right] d y .
$$

Logo,

$$
V(t, y)=\int M(t, y) d t+\int\left[N(t, y)-\int \frac{\partial M}{\partial y}(t, y) d t\right] d y
$$

é uma função tal que $\frac{\partial V}{\partial t}=M \mathrm{e} \frac{\partial V}{\partial y}=N$. 
Note que a demonstração do Teorema 2 nos proporciona um método para calcularmos $V(t, y)$ e, desse modo, a solução da equação (3.10). Veja ainda que é mais fácil repetir o processo realizado no Teorema 2 do que utilizar a expressão de $V(t, y)$ obtida neste Teorema. Observamos ainda que as soluções são dadas, na maioria dos casos, de forma implícita e nem sempre será possível encontrá-las na forma explícita.

Exemplo 18. Encontremos a solução do P.V.I.

$$
\left\{\begin{array}{l}
\left(2 t^{3}+3 y\right) d t+(3 t+y-1) d y=0 \\
y(0)=4
\end{array}\right.
$$

Neste caso, temos que $M(t, y)=2 t^{3}+3 y$ e $N(t, y)=3 t+y-1$. Esta equação é exata, pois $\frac{\partial M}{\partial y}=3=\frac{\partial N}{\partial t} \mathrm{e}$, assim, existe uma função $V(t, y)$ tal que

$$
\text { (i) } \quad \frac{\partial V}{\partial t}(t, y)=2 t^{3}+3 y \quad \text { e } \quad(i i) \quad \frac{\partial V}{\partial y}(t, y)=3 t+y-1
$$

Integrando $(i)$ em $t$ obtemos

$$
V(t, y)=\int\left(2 t^{3}+3 y\right) d t+h(y)=\frac{t^{4}}{2}+3 t y+h(y) .
$$

Derivando agora em relação a $y$ e usando $(i i)$ temos

$$
3 t+h^{\prime}(y)=\frac{\partial V}{\partial y}=3 t+y-1 \Rightarrow h^{\prime}(y)=y-1 \Rightarrow h(y)=\frac{y^{2}}{2}-y .
$$

Assim, as curvas integrais são dadas por

$$
V(t, y)=\frac{t^{4}}{2}+3 t y+\frac{y^{2}}{2}-y=c .
$$

Aplicando a condição inicial do P.V.I., ou seja, $y(0)=4$, obtemos

$$
\frac{0^{2}}{2}+3 \cdot 0 \cdot 4+\frac{4^{2}}{2}-4=c \Rightarrow c=4
$$

Logo, a solução do P.V.I. é dada implicitamente por

$$
\frac{t^{4}}{2}+3 t y+\frac{y^{2}}{2}-y=4
$$

Observação 17. É interessante observar que embora tenhamos começado o processo por $V(t, y)=\int M(t, y) d t+h(y)$, poderíamos ter começado por $V(t, y)=\int N(t, y) d y+g(t)$ e prosseguido de maneira similar ao que foi feito na demonstração do Teorema 2.

Para ilustrar a observação acima, veja que no Exemplo 18 podemos começar o processo integrando (ii) em y e ficando com

$$
V(t, y)=\int(3 t+y-1) d y+g(t)=3 t y+\frac{y^{2}}{2}-y+g(t) .
$$


Derivando agora com relação a $t$ e usando $(i)$ temos

$$
3 y+g^{\prime}(t)=\frac{\partial V}{\partial t}=2 t^{3}+3 y \Rightarrow g^{\prime}(t)=2 t^{3} \Rightarrow g(t)=\frac{t^{4}}{2} .
$$

Assim, as curvas integrais são dadas por

$$
V(t, y)=\frac{t^{4}}{2}+3 t y+\frac{y^{2}}{2}-y=c
$$

que é a mesma função encontrada anteriormente.

\subsubsection{Equações com Variáveis Separáveis}

Definição 15. Uma equação com variáveis separáveis é uma equação da forma

$$
M(t) N(y) d t+P(t) Q(y) d y=0
$$

onde $P(t) \neq 0$ para todo $t$ e $N(y) \neq 0$ para todo $y$.

Note que quando multiplicamos (3.13) por $\mu(t, y)=\frac{1}{P(t) N(y)}$ obtemos

$$
\frac{M(t)}{P(t)} d t+\frac{Q(y)}{N(y)} d y=0
$$

o que justifica o nome dado para estas equações.

É claro que (3.14) é uma equação exata, uma vez que

$$
\frac{\partial}{\partial y}\left(\frac{M(t)}{P(t)}\right)=0=\frac{\partial}{\partial t}\left(\frac{Q(y)}{N(y)}\right) .
$$

Logo, as curvas integrais são dadas por

$$
V(t, y)=\int \frac{M(t)}{P(t)} d t+\int \frac{Q(y)}{N(y)} d y=c,
$$

que definem implicitamente a solução $y(t)$ de (3.13). De fato, se $V(t, y)$ é tal que

$$
\frac{\partial V}{\partial t}=\frac{M(t)}{P(t)} \quad \text { e } \quad \frac{\partial V}{\partial y}=\frac{Q(y)}{N(y)},
$$

integrando a primeira equação em $t$ temos que

$$
V(t, y)=\int \frac{M(t)}{P(t)} d t+h(y) .
$$

Derivando esta equação em $y$ e substituindo $\frac{\partial V}{\partial y}(t, y)=\frac{Q(y)}{N(y)}$ ficamos com

$$
\begin{aligned}
\frac{Q(y)}{N(y)} & =\frac{\partial}{\partial y}\left[\int \frac{M(t)}{P(t)} d t\right]+h^{\prime}(y) \\
& =0+h^{\prime}(y) \\
& =h^{\prime}(y)
\end{aligned}
$$


o que implica que $h(y)=\int \frac{Q(y)}{N(y)} d y$ e, portanto, que

$$
V(t, y)=\int \frac{M(t)}{P(t)} d t+\int \frac{Q(y)}{N(y)} d y .
$$

Exemplo 19. Encontremos a solução do P.V.I.

$$
\left\{\begin{array}{l}
y^{\prime}+e^{-y} \cos t=0 \\
y(0)=1
\end{array}\right.
$$

Note que podemos escrever a equação deste P.V.I. na forma

$$
1 d y+e^{-y} \cos t d t=0
$$

que é uma equação com variáveis separáveis onde $M(t)=\cos t, N(y)=e^{-y}, P(t)=1$ e $Q(y)=1$. Assim, $\mu(t, y)=\frac{1}{1 e^{-y}}$ e multiplicando a equação por $\mu(t, y)$ ficamos com

$$
e^{y} d y+\cos t d t=0
$$

Logo, as curvas integrais da equação são dadas por

$$
\begin{aligned}
V(t, y) & =\int e^{y} d y+\int \cos t d t \\
& =e^{y}+\operatorname{sen} t \\
& =c,
\end{aligned}
$$

o que implica que

$$
e^{y}=c-\operatorname{sen} t
$$

Como $y(0)=1$, concluímos que

$$
e^{1}=c-\operatorname{sen} 0 \Rightarrow c=e
$$

Portanto, a solução do P.V.I. é dada por

$$
y(t)=\ln (e-\operatorname{sen} t) .
$$

Exemplo 20. Encontremos a solução do P.V.I.

$$
\left\{\begin{array}{l}
\left(1+t^{3}\right) d y-3 t^{2} y d t=0, \\
y(1)=4
\end{array}\right.
$$

Note primeiro que, como no P.V.I. dado temos a condição inicial $y(1)=4$, podemos assumir que estamos procurando uma solução em torno do ponto $(1, y(1))=(1,4)$. Especificamente, podemos assumir que $t, y>0$. Assim, temos uma equação com variáveis separáveis, onde 
$M(t)=\left(-3 t^{2}\right), N(y)=y, P(t)=\left(1+t^{3}\right)$ e $Q(y)=1$. Assim, $\mu(t, y)=\frac{1}{\left(1+t^{3}\right) y}$ e multiplicando a equação por $\mu(t, y)$ ficamos com

$$
\frac{d y}{y}-\frac{3 t^{2}}{1+t^{3}} d t=0
$$

Logo, as curvas integrais da equação são dadas por

$$
\begin{aligned}
V(t, y) & =\int \frac{d y}{y}-\int \frac{3 t^{2}}{1+t^{3}} d t \\
& =\ln |y|-\ln \left|1+t^{3}\right| \\
& =\ln y-\ln \left(1+t^{3}\right) \\
& =c,
\end{aligned}
$$

o que implica que

$$
\ln y=c+\ln \left(1+t^{3}\right)
$$

e, assim

$$
y(t)=e^{c} \cdot\left(1+t^{3}\right) .
$$

Como $y(1)=4$, concluímos que

$$
4=e^{c} \cdot 2 \Rightarrow e^{c}=2 .
$$

Portanto, a solução do P.V.I. é dada por

$$
y(t)=2\left(1+t^{3}\right) .
$$

\subsubsection{Equações Autômomas}

Uma equação autônoma é aquela em que a variável independente não aparece explicitamente. Essas equações têm a forma

$$
\frac{d y}{d t}=f(y)
$$

É fácil observar que uma equação autônoma é uma equação de variáveis separáveis, desde que $f(y) \neq 0$ para todo $y$.

Exemplo 21. Resolva a equação $\frac{d y}{d t}=2 y^{2}$, onde $y(t) \neq 0$ para todo $t \in I$.

Temos que essa equação pode ser reescrita como $\frac{d y}{2 y^{2}}-d t=0$, e as curvas integrais da equação são dadas por

$$
\int \frac{d y}{2 y^{2}}-\int d t=c_{1}
$$

de onde concluímos que

$$
-\frac{1}{2 y}=c_{1}+t
$$

Portanto, a solução da equação é dada por

$$
y(t)=\frac{1}{2\left(-c_{1}-t\right)}=\frac{1}{c-2 t} .
$$




\subsubsection{Equações Homogêneas}

Definição 16. Dizemos que uma função $f: D \subset \mathbb{R}^{2} \rightarrow \mathbb{R}$ é homogênea de grau $n$ se

$$
f(\lambda t, \lambda y)=\lambda^{n} f(t, y)
$$

para todo $\lambda \neq 0$ e todo $(t, y) \in D$ tais que $(\lambda t, \lambda y) \in D$.

Exemplo 22. A função $f(t, y)=t^{2}-t y$ é homogênea de grau 2, pois

$$
f(\lambda t, \lambda y)=(\lambda t)^{2}-(\lambda t)(\lambda y)=\lambda^{2} t^{2}-\lambda^{2} t y=\lambda^{2}\left(t^{2}-t y\right)=\lambda^{2} f(t, y) .
$$

Definição 17. Dizemos que a equação $M(t, y)+N(t, y) y^{\prime}=0$ é homogênea se $M(t, y)$ e $N(t, y)$ são funções homogêneas de mesmo grau.

Exemplo 23. A equação $(4 t-3 y)+(2 y-3 t) y^{\prime}=0$ é homogênea de grau 1 . De fato, neste caso, $M(t, y)=4 t-3 y \mathrm{e}$

$$
M(\lambda t, \lambda y)=4 \lambda t-3 \lambda y=\lambda(4 t-3 y)=\lambda M(t, y) .
$$

Do mesmo modo, $N(t, y)=2 y-3 t \mathrm{e}$

$$
N(\lambda t, \lambda y)=2 \lambda y-3 \lambda t=\lambda(2 y-3 t)=\lambda N(t, y) .
$$

Exemplo 24. A equação $\left(t^{2}+y^{2}\right)+\left(t^{3}-y^{3}\right) y^{\prime}=0$ não é homogênea. De fato, neste caso, $M(t, y)=t^{2}+y^{2} \mathrm{e}$

$$
M(\lambda t, \lambda y)=\lambda^{2} t^{2}+\lambda^{2} y^{2}=\lambda^{2}\left(t^{2}+y^{2}\right)=\lambda^{2} M(t, y) .
$$

Por outro lado, $N(t, y)=t^{3}-y^{3} \mathrm{e}$

$$
N(\lambda t, \lambda y)=\lambda^{3} t^{3}-\lambda^{3} y^{3}=\lambda^{3}\left(t^{3}-y^{3}\right)=\lambda^{3} N(t, y) .
$$

Logo, embora $M(t, y)$ e $N(t, y)$ sejam homogêneas, elas não são de mesmo grau. Portanto, a equação não é homogênea.

Exemplo 25. A equação $(t+3 y)+\left(2 t-y^{2}\right) y^{\prime}=0$ não é homogênea. De fato, temos que $M(t, y)=t+3 y \mathrm{e}$

$$
M(\lambda t, \lambda y)=\lambda t+3 \lambda y=\lambda(t+3 y)=\lambda M(t, y) .
$$

Por outro lado, $N(t, y)=2 t-y^{2} \mathrm{e}$

$$
N(\lambda t, \lambda y)=2 \lambda t-\lambda^{2} y^{2}
$$

o que implica que $N(t, y)$ não é homogênea e, portanto, a equação não é homogênea. 
Para resolver a equação homogênea fazemos a mudança de variáveis $y(t)=t v(t) \mathrm{e}$, consequentemente, temos

$$
d y=v d t+t d v
$$

Veremos que esta mudança de variáveis transformará a equação homogênea em uma equação com variáveis separáveis.

Partindo de $M(t, y)+N(t, y) y^{\prime}=0$ temos $y^{\prime}=-\frac{M(t, y)}{N(t, y)}$, ou seja,

$$
d y=-\frac{M(t, y)}{N(t, y)} d t
$$

Portanto, supondo que a equação é homogênea de grau $n$ e usando a mudança de variáveis acima, para $t \neq 0$ e $(1, v)$ pertencente ao domínio de $M$ e $N$, temos que

$$
\begin{aligned}
v d t+t d v & =-\frac{M(t \cdot 1, t \cdot v)}{N(t \cdot 1, t \cdot v)} d t \\
& =-\frac{t^{n} M(1, v)}{t^{n} N(1, v)} d t \\
& =-\frac{M(1, v)}{N(1, v)} d t
\end{aligned}
$$

ou seja,

$$
\left[v+\frac{M(1, v)}{N(1, v)}\right] d t+t d v=0,
$$

que é equivalente à seguinte equação com variáveis separáveis

$$
\frac{1}{t} d t+\frac{1}{v+\frac{M(1, v)}{N(1, v)}} d v=0 .
$$

Exemplo 26. Encontremos a solução do P.V.I.

$$
\left\{\begin{array}{l}
(4 t-3 y)+(2 y-3 t) y^{\prime}=0 \\
y(1)=3
\end{array}\right.
$$

Sabemos, do exemplo 23, que esta equação é homogênea. Assim, fazendo a substiuição $y=t v$ temos que ela pode ser reescrita como

$$
(4 t-3 t v) d t+(2 t v-3 t)(v d t+t d v)=0
$$

e reorganizando as derivadas temos

$$
\left(4-6 v+2 v^{2}\right) t d t+(2 v-3) t^{2} d v=0
$$

Separando as variáveis obtemos

$$
\frac{1}{t} d t+\frac{2 v-3}{2 v^{2}-6 v+4} d v=0 .
$$


Integrando ambos os membros, para encontrarmos as curvas integrais, ficamos com

$$
\int \frac{1}{t} d t+\int \frac{2 v-3}{2 v^{2}-6 v+4} d v=c
$$

Ou seja,

$$
\ln |t|+\frac{1}{2} \ln \left|v^{2}-3 v+2\right|=c
$$

o que implica que

$$
\ln \left|v^{2}-3 v+2\right|^{\frac{1}{2}}=c-\ln |t|
$$

Logo,

$$
\begin{aligned}
\left|v^{2}-3 v+2\right|^{\frac{1}{2}} & =e^{(c-\ln |t|)} \\
& =e^{c} \cdot e^{-\ln |t|} \\
& =e^{c} \cdot \frac{1}{|t|} .
\end{aligned}
$$

Utilizando a substituição $v=\frac{y}{t}$, concluímos que a solução da equação é dada implicitamente por

$$
\left|\frac{y^{2}}{t^{2}}-\frac{3 y}{t}+2\right|^{\frac{1}{2}}=\frac{e^{c}}{|t|} .
$$

Usando agora a condição inicial $y(1)=3$ obtemos

$$
|9-9+2|^{\frac{1}{2}}=e^{c} \Rightarrow e^{c}=\sqrt{2} .
$$

Logo, observando que podemos supor $t>0$, a solução do P.V.I. é dada implicitamente por

$$
\left|\frac{y^{2}}{t^{2}}-\frac{3 y}{t}+2\right|^{\frac{1}{2}}=\frac{\sqrt{2}}{t} .
$$

Observação 18. Como vimos no Exemplo 26, em geral, é mais fácil repetir o processo do que decorar a equação (3.15).

\subsubsection{Fatores Integrantes}

Suponha que a equação diferencial

$$
M(t, y)+N(t, y) y^{\prime}=0
$$

não seja exata, com variáveis separáveis ou homogênea. Então, para resolvê-la tentaremos encontrar uma função $\mu(t, y)$, chamada de fator integrante de (3.16), tal que

$$
\mu(t, y) M(t, y)+\mu(t, y) N(t, y) y^{\prime}=0
$$

seja uma EDO exata. 
Geralmente, é difícil determinarmos fatores integrantes, pois $\mu(t, y)$ é fator integrante de (3.16) se, e somente se,

$$
\frac{\partial(\mu M)}{\partial y}=\frac{\partial(\mu N)}{\partial t}
$$

ou seja,

$$
M \frac{\partial \mu}{\partial y}+\mu \frac{\partial M}{\partial y}=N \frac{\partial \mu}{\partial t}+\mu \frac{\partial N}{\partial t}
$$

que não é uma equação fácil de trabalhar. A seguir, veremos uma classe de EDOs cujo fator integrante pode ser encontrado com facilidade.

Veja que, se for possível encontrar um fator integrante para (3.16) que só dependa de $t$, então a equação

$$
\mu(t) M(t, y)+\mu(t) N(t, y) y^{\prime}=0
$$

é exata. Logo,

$$
\frac{\partial}{\partial y}(\mu(t) M(t, y))=\frac{\partial}{\partial t}(\mu(t) N(t, y))
$$

isto é,

$$
M \frac{\partial \mu(t)}{\partial y}+\mu(t) \frac{\partial M}{\partial y}=N \frac{d \mu(t)}{d t}+\mu(t) \frac{\partial N}{\partial t}
$$

Assim,

$$
\frac{d \mu(t)}{d t}=\frac{1}{N}\left(\frac{\partial M}{\partial y}-\frac{\partial N}{\partial t}\right) \mu(t)
$$

Observe agora que a equação acima só tem sentido quando $\frac{1}{N}\left(\frac{\partial M}{\partial y}-\frac{\partial N}{\partial t}\right)$ for uma função que só depende de $t$, ou seja,

$$
\frac{1}{N}\left(\frac{\partial M}{\partial y}-\frac{\partial N}{\partial t}\right)=f(t)
$$

Desse modo, temos

$$
\frac{d \mu(t)}{d t}=f(t) \mu(t)
$$

que é uma EDO linear homogênea de primeira ordem, cuja solução é dada por

$$
\mu(t)=e^{\int f(t) d t} .
$$

Analogamente, verificamos que se $\mu$ depender somente de $y$ teremos

$$
\frac{1}{M}\left(\frac{\partial N}{\partial t}-\frac{\partial M}{\partial y}\right)=g(y)
$$

e, então, $\mu(y)=e^{\int g(y) d y}$ será um fator integrante de (3.16).

Exemplo 27. Encontremos um fator integrante para a equação

$$
\left(3 t^{2} y+2 t y+y^{3}\right) d t+\left(t^{2}+y^{2}\right) d y=0 .
$$


Neste caso, temos que $M(t, y)=3 t^{2} y+2 t y+y^{3}$ e, então, $\frac{\partial M}{\partial y}=3 t^{2}+2 t+3 y^{2}$. Além disso, $N(t, y)=t^{2}+y^{2}$ e, então, $\frac{\partial N}{\partial t}=2 t$. Logo, notando que

$$
\frac{1}{N}\left(\frac{\partial M}{\partial y}-\frac{\partial N}{\partial t}\right)=\frac{3\left(t^{2}+y^{2}\right)}{t^{2}+y^{2}}=3=f(t),
$$

podemos procurar um fator integrante da forma $\mu(t)$, tal que

$$
\mu(t)\left(3 t^{2} y+2 t y+y^{3}\right) d t+\mu(t)\left(t^{2}+y^{2}\right) d y=0
$$

seja uma equação exata, ou seja,

$$
\frac{\partial}{\partial y}\left(\mu(t)\left(3 t^{2} y+2 t y+y^{3}\right)\right)=\frac{\partial}{\partial t}\left(\mu(t)\left(t^{2}+y^{2}\right)\right) .
$$

Procedendo como acima, chegamos na seguinte EDO linear homogênea de primeira ordem

$$
\mu^{\prime}(t)=3 \mu(t)
$$

Resolvendo esta EDO, teremos que um fator integrante para a equação diferencial é

$$
\mu(t)=e^{\int 3 d t}=e^{3 t} .
$$

Observação 19. Note que no Exemplo 27 não seria conveniente procurarmos um fator integrante da forma $\mu(y)$, pois

$$
\frac{1}{M}\left(\frac{\partial N}{\partial t}-\frac{\partial M}{\partial y}\right)=\frac{-3 t^{2}-3 y^{2}}{3 t^{2}+2 t y+y^{3}} \neq g(y) .
$$

Exemplo 28. Encontremos um fator integrante para a equação $y d t+\left(2 t y-e^{-2 y}\right) d y=0$.

Neste caso, temos que $M(t, y)=y$ e, então, $\frac{\partial M}{\partial y}=1$, e $N(t, y)=2 t y-e^{-2 y}$ e, então, $\frac{\partial N}{\partial t}=2 y$. Então, observando que

$$
\frac{1}{M}\left(\frac{\partial N}{\partial t}-\frac{\partial M}{\partial y}\right)=\frac{2 y-1}{y}=g(y)
$$

podemos procurar um fator integrante da forma $\mu(y)$, tal que

$$
\mu(y) y d t+\mu(y)\left(2 t y-e^{-2 y}\right) d y=0
$$

seja uma equação exata, ou seja,

$$
\frac{\partial}{\partial y}(\mu(y) y)=\frac{\partial}{\partial t}\left(\mu(y)\left(2 t y-e^{-2 y}\right)\right),
$$

de onde obtemos

$$
\mu^{\prime}(y)=\left(\frac{2 y-1}{y}\right) \mu(y) .
$$


Resolvendo esta EDO vemos que

$$
\mu(y)=e^{\int \frac{2 y-1}{y} d y}=e^{\int\left[2-\frac{1}{y}\right] d y}=e^{2 y-\ln |y|}=\frac{e^{2 y}}{|y|} .
$$

Logo, um fator integrante para a equação dada é, por exemplo,

$$
\mu(y)=\frac{e^{2 y}}{y} .
$$

Observação 20. Novamente, veja que no Exemplo 28 não seria conveniente procurarmos um fator integrante da forma $\mu(t)$, pois

$$
\frac{1}{N}\left(\frac{\partial M}{\partial y}-\frac{\partial N}{\partial t}\right)=\frac{1-2 y}{2 t y-e^{-2 y}} \neq f(t) .
$$

Observação 21. Veja que nos exemplos acima não nos preocupamos com a constante de integração, pois escolhemos "uma" dentre todas as funções $\mu$ que tornam a equação exata.

\subsubsection{Algumas Aplicações}

\subsubsection{Modelo Populacional de Verhulst}

Como já foi visto, a taxa de variação populacional segundo o modelo de Verhulst é dada pela EDO

$$
p^{\prime}(t)=k p(t)\left(1-\frac{p(t)}{C}\right)
$$

Ou ainda,

$$
\frac{d p}{d t}=k p\left(1-\frac{p}{C}\right)
$$

onde $k$ representa a taxa de crescimento na falta de fator limitante e $C>0$ é a capacidade de carga populacional suportada pelo ambiente em que a população vive.

Note que esta EDO, desde que $p(t) \neq 0$ e $p(t) \neq C$, é uma equação com variáveis separáveis e pode ser reescrita da forma

$$
\frac{1}{p\left(1-\frac{p}{C}\right)} d p=k d t
$$

Logo, podemos obter sua solução integrando ambos os membros da equação. A integral do primeiro membro é encontrada pelo método das frações parciais. Especificamente, temos que

$$
\frac{1}{p\left(1-\frac{p}{C}\right)}=\frac{A}{p}+\frac{B}{\left(1-\frac{p}{C}\right)}=\frac{A\left(1-\frac{p}{C}\right)+B p}{p\left(1-\frac{p}{C}\right)} .
$$

Logo, podemos concluir que

$$
1=A\left(1-\frac{p}{C}\right)+B p
$$


o que implica que $1=\left(B-\frac{A}{C}\right) p+A$. Daí segue que devemos ter $A=1$ e $B=\frac{1}{C}$. Logo,

$$
\frac{1}{p\left(1-\frac{p}{C}\right)}=\frac{1}{p}+\frac{\frac{1}{C}}{\left(1-\frac{p}{C}\right)} .
$$

Então,

$$
\begin{aligned}
\int \frac{1}{p\left(1-\frac{p}{C}\right)} d p & =\int \frac{1}{p} d p+\int \frac{\frac{1}{C}}{\left(1-\frac{p}{C}\right)} d p \\
& =\ln |p|-\ln \left|1-\frac{p}{C}\right| \\
& =\ln \left|\frac{p}{1-\frac{p}{C}}\right| .
\end{aligned}
$$

Logo, integrando a equação (3.17) obtemos

$$
\ln \left|\frac{p}{1-\frac{p}{C}}\right|=k t+c_{1}
$$

o que implica que

$$
\begin{aligned}
\left|\frac{p}{1-\frac{p}{C}}\right| & =e^{c_{1}} e^{k t} \\
& :=c e^{k t}
\end{aligned}
$$

Logo, se $p(0)=p_{0}$, com $p_{0}>0$ e $p_{0} \neq C$, temos que

$$
\frac{p_{0}}{\left|1-\frac{p_{0}}{C}\right|}=c
$$

o que implica que $c=\frac{p_{0} C}{C-p_{0}}$, para $p_{0}<C$, e $c=\frac{p_{0} C}{p_{0}-C}$, para $p_{0}>C$. Considerando estas duas situações obtemos as seguintes conclusões:

I) Para $0<p_{0}<C$, podemos verificar que $0<p(t)<C$ para qualquer $t$ e este fato será explicado mais adiante. Então,

$$
p(t)=\left|1-\frac{p(t)}{C}\right| e^{k t} \frac{p_{0} C}{C-p_{0}}=\left(1-\frac{p(t)}{C}\right) \cdot e^{k t} \frac{p_{0} C}{C-p_{0}}=e^{k t} \frac{p_{0} C}{C-p_{0}}-p(t) e^{k t} \frac{p_{0}}{C-p_{0}},
$$

o que implica que

$$
p(t)=\frac{C e^{k t} p_{0}}{C-p_{0}+p_{0} e^{k t}} .
$$

II) Para $p_{0}>C$, podemos verificar que $p(t)>C$ para qualquer $t$ e este fato será também explicado mais adiante. Então,

$$
p(t)=\left|1-\frac{p(t)}{C}\right| e^{k t} \frac{p_{0} C}{p_{0}-C}=\left(\frac{p(t)}{C}-1\right) \cdot e^{k t} \frac{p_{0} C}{p_{0}-C}=p(t) e^{k t} \frac{p_{0}}{p_{0}-C}-e^{k t} \frac{p_{0} C}{p_{0}-C},
$$


o que implica que

$$
p(t)=\frac{C e^{k t} p_{0}}{C-p_{0}+p_{0} e^{k t}}
$$

Note que obtemos a mesma equação para $p(t)$ nos dois casos considerados acima, a qual é equivalente a

$$
p(t)=\frac{C p_{0}}{p_{0}+\left(C-p_{0}\right) e^{-k t}}
$$

Facilmente, da equação (3.18), vemos que para $t$ suficientemente grande, a população tende à constante $C$, ou seja, à capacidade de carga populacional suportada pelo ambiente.

Note que, além da solução encontrada acima, tanto $p_{1}(t)=0$ quanto $p_{2}(t)=C$ são soluções da equação de Verhulst e são chamadas soluções de equilíbrio, pois não há variação em $p(t)$ quando $t$ cresce, ou seja, a população não tem sua quantidade alterada.

Agora, note que, como a equação de Verhulst está de acordo com as condições do Teorema de Existência e Unicidade, então em cada ponto existe uma única solução. Desse modo, quando a população inicial encontra-se entre 0 e $C$, a solução para este caso deverá estar entre 0 e $C$. Assim, podemos afirmar que para $0<p_{0}<C$, temos $0<p(t)<C$ para qualquer $t$ e, do mesmo modo, temos que quando a população inicial encontra-se acima de $C$, a solução para este caso deverá estar acima de $C$ e, por consequência, podemos afirmar que para $p_{0}>C$, temos $p(t)>C$ para qualquer $t$.

Das considerações acima e de alguns argumentos de Cálculo Diferencial vemos que os gráficos das soluções da equação (3.17) podem ser ilustradas da seguinte forma

Figura 2 - Comportamento das soluções de (3.17)

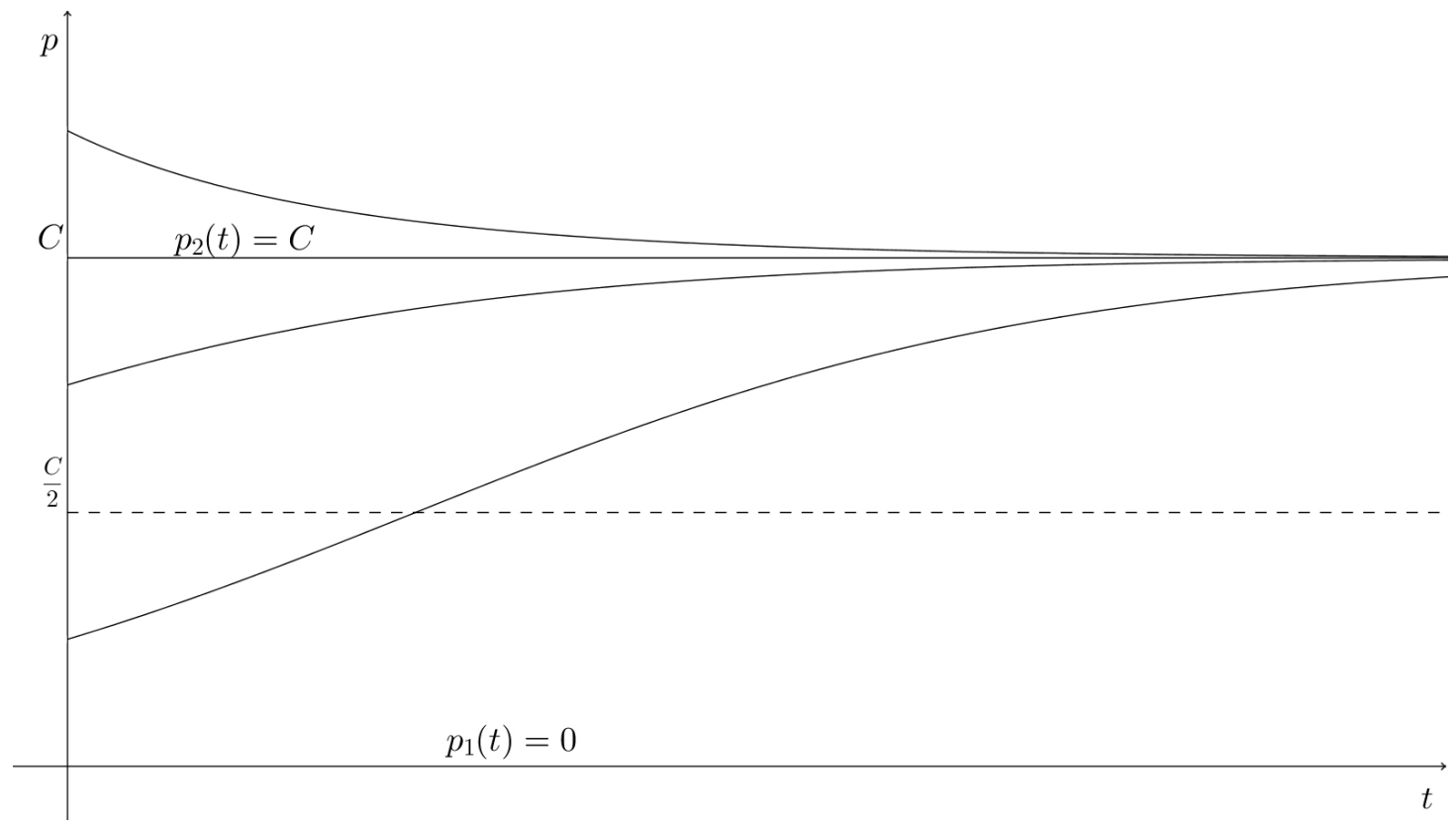

Fonte: Elaborada pelo autor. 
Exemplo 29. Suponha que o modelo populacional de Verhulst será aplicado a uma população de linguados gigantes em determinada área do Oceano Pacífico. Para isso, considere que $p(t)$ seja a massa total, em quilogramas, da população de linguados gigantes no instante $t, k=0,71$ ao ano e $C=80,5 \cdot 10^{6} \mathrm{~kg}$. Considerando ainda que a massa total inicial seja $p_{0}=0,25 C$, qual será a massa total depois de 2 anos?

Substituindo os valores na equação (3.18) obtemos

$$
\begin{aligned}
p(2) & =\frac{C 0,25 C}{0,25 C+(C-0,25 C) e^{-0,71 \cdot 2}} \\
& \cong \frac{0,25 C^{2}}{0,4313 C} \\
& \cong 0,5797 C \\
& \cong 46,67 \cdot 10^{6} .
\end{aligned}
$$

Assim, a massa total da população de linguados gigantes, após 2 anos, será aproximadamente $46,67 \cdot 10^{6} \mathrm{~kg}$.

Exemplo 30. Considerando os dados fornecidos no Exemplo 29, calculemos o instante $t$ no qual $p(t)=0,75 C \mathrm{~kg}$. Para isso, basta substituir $p(t)=0,75 C$ na Equação (3.18), ou seja,

$$
\begin{aligned}
0,75 C & =\frac{C 0,25 C}{0,25+(C-0,25 C) e^{-0,71 t}} \\
0,75 & =\frac{1}{1+3 e^{-0,71 t}} \\
e^{-0,71 t} & =\frac{0,25}{2,25} \\
e^{0,71 t} & =9 \\
0,71 t & =\ln 9 \\
t & \cong 3,095 .
\end{aligned}
$$

Logo, a massa total da população de linguados gigantes atingirá $0,75 \mathrm{C}$ em aproximadamente 3,095 anos, ou seja, em aproximadamente 3 anos e 1 mês.

Exemplo 31. Uma pessoa infectada com o vírus da gripe vai para uma ilha isolada onde habitam outras 999 pessoas, totalizando, então, 1000 pessoas. Suponha que a taxa na qual o vírus se espalha é proporcional tanto à quantidade de pessoas infectadas quanto à de não infectadas. Determine quantas pessoas estarão infectadas após 6 dias sabendo que depois de 4 dias havia 50 pessoas infectadas e que ninguém saiu da ilha.

Considerando $p$ como a quantidade de pessoas infectadas temos que a situação pode ser modelada pela equação

$$
\frac{d p}{d t}=K p(1000-p),
$$

que é equivalente a

$$
\frac{d p}{d t}=1000 K p\left(1-\frac{p}{1000}\right)
$$


que é similar à equação do modelo populacional de Verhulst, onde $k=1000 \mathrm{~K}$ e $C=1000$. Assim, considerando $p(0)=1$, a solução da equação que modela o problema é dada por

$$
p(t)=\frac{1000}{1+999 e^{-1000 K t}} .
$$

Como após 4 dias havia 50 pessoas infectadas, temos que

$$
50=\frac{1000}{1+999 e^{-4000 K}} \Rightarrow K \cong 0,0009906 \Rightarrow 1000 K \cong 0,9906 .
$$

Logo, substitiuindo $1000 K=0,9906$ na última equação, ficamos com

$$
p(t)=\frac{1000}{1+999 e^{-0,9906 t}},
$$

e, então,

$$
p(6)=\frac{1000}{1+999 e^{-0,9906(6)}} \cong 276 .
$$

Assim, temos que após 6 dias aproximadamente 276 pessoas estarão infactadas.

\section{Limiar Crítico}

A seguir apresentamos, brevemente, a ideia do conceito de limiar crítico, com o objetivo de relacionar este conceito com o modelo de Verhulst. Para isso, consideremos a equação

$$
\frac{d p}{d t}=-k p\left(1-\frac{p}{T}\right)
$$

onde $k$ e $T$ são constantes positivas. Note que esta equação difere da Equação de Verhulst apenas pelo sinal negativo no lado direito da igualdade e na substituição da constante $C$ pela constante $T$. No entanto, veremos que o comportamento das soluções da equação (3.19) é bem diferente das soluções da Equação de Verhulst.

Observamos, inicialmente, que $\frac{d p}{d t}=0$ se, e somente se, $p=0$ ou $p=T$, correspondendo às soluções de equilíbrio $p_{1}(t)=0$ e $p_{2}(t)=T$.

Agora, se $0<p<T$, então $\frac{d p}{d t}<0$, o que implica que $p$ é decrescente como função de $t$. Por outro lado, se $p>T$, então $\frac{d p}{d t}>0$ e $p$ é crescente como função de $t$.

De maneira mais específica, utilizando o método de separação de variáveis obtemos, de forma análoga ao que foi feito para a Equação de Verhulst, que a solução de (3.19) com $p(0)=p_{0}$ é dada por

$$
p(t)=\frac{T p_{0}}{p_{0}+\left(T-p_{0}\right) e^{k t}} .
$$

Logo, se $0<p_{0}<T$, vemos facilmente que $p(t) \rightarrow 0$ quando $t \rightarrow \infty$. Por outro lado, se $p_{0}>T$, então o denominador do lado direito de (3.20) se anula para algum valor finito de $t$. Seja $t^{*}$ esse valor. Então

$$
p_{0}+\left(T-p_{0}\right) e^{k t^{*}}=0
$$


o que implica que

$$
t^{*}=\frac{1}{k} \ln \frac{p_{0}}{p_{0}-T}
$$

Logo, $\lim _{t \rightarrow t_{-}^{*}} p(t)=\infty$, ou seja, se a população inicial está acima de $T$, o gráfico de $p$ em função de $t$ tem uma assíntota vertical em $t=t^{*}$. Ou seja, a população se torna ilimitada em um tempo finito que depende de $p_{0}, T$ e $k$.

Do estudo acima e de alguns argumentos de Cálculo Diferencial, podemos esboçar o comportamento das soluções de (3.19) da seguinte forma

Figura 3 - Comportamento das soluções de (3.19)

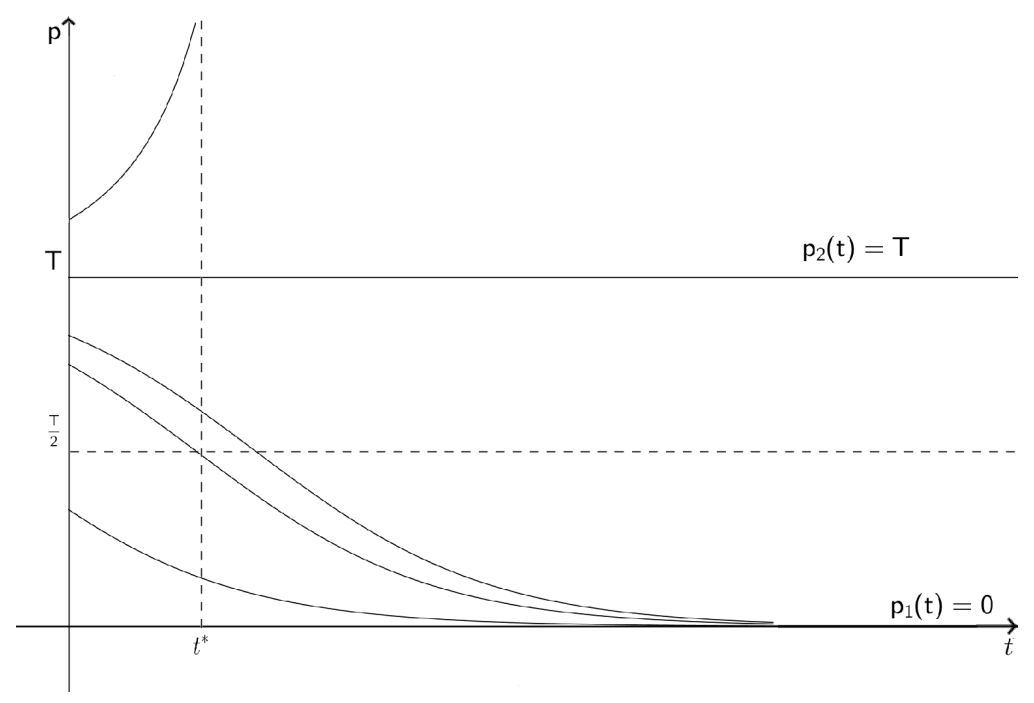

Fonte: Elaborada pelo autor.

Dizemos que $T$, no estudo acima, é um limiar abaixo do qual não há crescimento.

Algumas populações apresentam o fenômeno de limiar, ou seja, se existem poucos indivíduos, essa população tende a ser extinta. Entretanto, se for possível reunir uma população maior que o limiar, então ocorrerá um crescimento ainda maior. Por outro lado, é impossível uma população tornar-se ilimitada. Logo, deve ser feita alguma modificação na equação (3.19) a fim de evitar este problema.

\section{Crescimento Logístico com Limiar}

Como dissemos acima, o modelo com limiar deve ser modificado para evitar crescimento ilimitado quando $p(t)$ está acima do limiar $T$. A maneira mais simples de modificar a equação (3.19), de modo que não ocorra um crescimento ilimitado, é introduzir um fator que tornará $\frac{d p}{d t}$ negativo para $p$ grande. Para isto, consideremos a equação

$$
\frac{d p}{d t}=-k p\left(1-\frac{p}{T}\right)\left(1-\frac{p}{C}\right)
$$


onde $k>0$ e $0<T<C$. A equação (3.21) é uma equação com variáveis separáveis e para encontrar uma solução integramos ambos os membros desta equação. Ou seja,

$$
\int \frac{d p}{p\left(1-\frac{p}{T}\right)\left(1-\frac{p}{C}\right)}=-\int k d t
$$

Pelo método das frações parciais, é fácil verificar que

$$
\frac{1}{p\left(1-\frac{p}{T}\right)\left(1-\frac{p}{C}\right)}=\frac{1}{p}+\frac{C}{(C-T)(T-p)}-\frac{T}{(C-T)(C-p)} .
$$

Portanto, para encontrar a solução da equação (3.21) basta resolver

$$
\int \frac{1}{p} d p+\int \frac{C}{(C-T)(T-p)} d p-\int \frac{T}{(C-T)(C-p)} d p=-\int k d t
$$

Resolvendo as integrais temos

$$
\ln |p|+\left(\frac{C}{C-T}\right) \ln |T-p|-\left(\frac{T}{C-T}\right) \ln |C-p|=-k t+\alpha_{1}
$$

que é equivalente a

$$
\ln \left|\frac{p(T-p)^{\frac{C}{C-T}}}{(C-p)^{\frac{T}{C-T}}}\right|=-k t+\alpha_{1} .
$$

Aplicando a exponencial obtemos

$$
\left|\frac{p(T-p)^{\frac{C}{C-T}}}{(C-p)^{\frac{T}{C-T}}}\right|=\alpha e^{-k t}
$$

ou seja, a solução da equação (3.21) é dada, implicitamente, pela equação

$$
\left|\frac{p(t)(T-p(t))^{\frac{C}{C-T}}}{(C-p(t))^{\frac{T}{C-T}}}\right|=\alpha e^{-k t}
$$

Note que (3.22) não é uma expressão fácil de trabalhar. Logo, para analisar e esboçar o comportamento dessas soluções usaremos a própria equação (3.21). Inicialmente, observe que $p_{1}(t)=0, p_{2}(t)=T$ e $p_{3}(t)=C$ são soluções de equilíbrio da equação (3.21). Por outro lado, é fácil ver que para $T<p_{0}<C$ devemos ter $T<p(t)<C$ para todo $t$ e, assim, $\frac{d p}{d t}>0$, o que implica que $p$ é crescente neste intervalo. Além disso, para $p_{0}<T$ ou $p_{0}>C$ devemos ter $p(t)<T$ ou $p(t)>C$ para todo $t$, respectivamente, e, assim, $\frac{d p}{d t}<0$, o que implica que $p$ é decrescente nestes intervalos. Logo, deste raciocínio e de alguns argumentos de Cálculo, podemos esboçar o comportamento das soluções da equação (3.21), como mostra a figura a seguir. 
Figura 4 - Comportamento das soluções de (3.21)

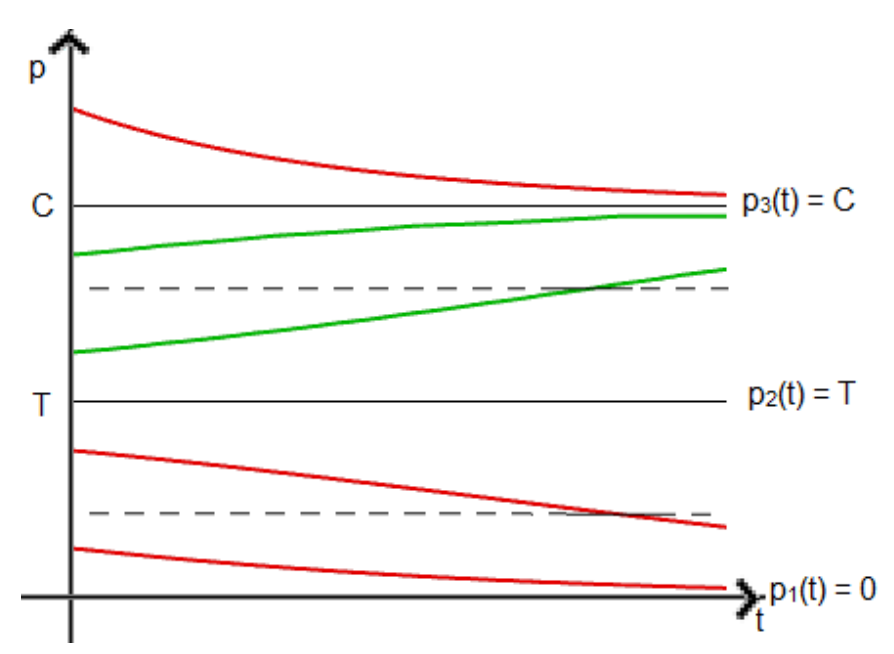

Fonte: Elaborada pelo autor.

Assim, da Figura 4, vemos que, se $p$ começa abaixo do limiar $T$, então $p$ decresce até chegar à sua extinção. Por outro lado, se $p$ começa acima de $T$, então $p$ vai se aproximando da capacidade de carga populacional $C$. Portanto, realmente, a equação (3.21) se mostra mais adequada que a equação (3.19).

Exemplo 32. Certa espécie de lêmure foi considerada em risco de extinção, portanto, com o intuito de salvar a espécie, foram reunidas 3200 lêmures dessa espécie em uma ilha. Após uma década sabe-se que a população é de 3503 lêmures. Sabendo que o limiar dessa espécie é de 2000 lêmures e a ilha tem capacidade de suportar 4000 lêmures, em quanto tempo a população de lêmures deverá se aproximar da capacidade da ilha?

Considerando a população dada em unidade de milhar e a unidade de tempo como década, temos $p(0)=3,2, p(1)=3,503, T=2$ e $C=4$ e o problema pode ser modelado pela equação

$$
\frac{d p}{d t}=-k p\left(1-\frac{p}{2}\right)\left(1-\frac{p}{4}\right)
$$

cuja solução é dada pela equação

$$
\left|\frac{p(t)(2-p(t))^{2}}{(4-p(t))}\right|=\alpha e^{-k t}
$$

Agora, usando que $p_{0}=3,2$ temos que

$$
\frac{3,2(2-3,2)^{2}}{4-3,2}=\alpha e^{0} \Rightarrow \alpha=5,76
$$

Da mesma forma, usando que $p(1)=3,503$ obtemos

$$
\frac{3,503(2-3,503)^{2}}{4-3,503}=0,64 e^{-k} \Rightarrow-k \cong 1,017 \text {. }
$$


Assim, temos que a solução da equação é dada por

$$
\frac{p(t)(2-p(t))^{2}}{(4-p(t))}=5,76 e^{1,017 t} .
$$

Logo, para saber em quanto tempo a população de lêmures deverá se aproximar da capacidade da ilha, basta substituir $p(t)$ por 3,999, ou seja,

$$
\frac{3,999(2-3,999)^{2}}{4-3,999}=5,76 e^{1,017 t} \Rightarrow t \cong 7,796 .
$$

Ou seja, a população de lêmures deverá se aproximar da capacidade da ilha em 7,796 décadas, ou seja, em aproximadamente 77 anos e 11,5 meses.

\subsubsection{Reação Química}

As reações químicas são transformações da matéria, que ocorrem como resultado da interação de certas substâncias, chamadas de reagentes, e o que temos após a reação são os produtos.

Quando se mistura substâncias que reagem entre si, não devemos esperar que tudo aconteça de imediato, pois é um processo em que os reagentes têm as ligações enfraquecidas e são formadas novas ligações.

Assim, sendo uma reação de síntese, ou de adição, aquela em que dois ou mais reagentes geram um único produto, temos que a variação da quantidade do produto final é proporcional à quantidade presente dos reagentes. Especificamente, considerando os reagentes $A$ e $B$ com quantidades, respectivamente, $\tilde{a}$ e $\tilde{b}$, o produto $Y$ com quantidade $y, a^{*}$ a porcentagem de $Y$ oriunda de $A, b^{*}$ a porcentagem de $Y$ oriunda de $B$, podemos modelar este problema da seguinte forma

$$
\frac{d y}{d t}=k_{1}\left(\tilde{a}-a^{*} y\right)\left(\tilde{b}-b^{*} y\right)
$$

que corresponde a

$$
\frac{d y}{d t}=k_{1} a^{*} b^{*}\left(\frac{\tilde{a}}{a^{*}}-y\right)\left(\frac{\tilde{b}}{b^{*}}-y\right) .
$$

Ou seja,

$$
\frac{d y}{d t}=k(a-y)(b-y),
$$

onde $k(>0)$ é uma constante, $a=\frac{\tilde{a}}{a^{*}}$ e $b=\frac{\tilde{b}}{b^{*}}$. Note que a equação (3.23) é uma equação com variáveis separáveis e podemos reescrevê-la na forma

$$
\frac{d y}{(a-y)(b-y)}=k d t \text {. }
$$

Agora, pelo método das frações parciais, é fácil verificar que

$$
\frac{1}{(a-y)(b-y)}=\frac{1}{(b-a)(a-y)}-\frac{1}{(b-a)(b-y)} \text {. }
$$


Logo, nossa equação corresponde a

$$
\frac{d t}{(b-a)(a-y)}-\frac{d t}{(b-a)(b-y)}=k d t .
$$

Integrando ambos os membros obtemos

$$
\frac{1}{(b-a)} \ln |(a-y)|-\frac{1}{(b-a)} \ln |(b-y)|=k t+c_{1},
$$

o que implica que

$$
\ln \left|\frac{a-y}{b-y}\right|=(b-a)\left(k t+c_{1}\right)
$$

e, portanto,

$$
\frac{a-y}{b-y}=e^{(b-a) k t} e^{(b-a) c_{1}} .
$$

Logo, tomando $c=e^{(b-a) c_{1}}$ a solução geral da equação (3.23) é dada por

$$
y(t)=\frac{b c e^{(b-a) k t}-a}{\left(c e^{(b-a) k t}-1\right)} .
$$

Agora, sabendo que no início da reação não há presença do produto $Y$, o que equivale a $y(0)=0$, temos que

$$
0=\frac{b c-a}{(c-1)} \Rightarrow c=\frac{a}{b}
$$

e, assim,

$$
y(t)=\frac{b c e^{(b-a) k t}-a}{\left(c e^{(b-a) k t}-1\right)}=\frac{b\left(\frac{a}{b}\right) e^{(b-a) k t}-a}{\left(\frac{a}{b}\right) e^{(b-a) k t}-1}=\frac{a b\left(e^{(b-a) k t}-1\right)}{a e^{(b-a) k t}-b} .
$$

Ou seja, a solução da equação (3.23) é dada por

$$
y(t)=\frac{a b\left(e^{(b-a) k t}-1\right)}{a e^{(b-a) k t}-b} .
$$

Exemplo 33. O ácido sulfúrico é um produto químico de alta importância, principalmente comercial, pois é utilizado como matéria-prima para diversos produtos como fertilizantes e detergentes. O ácido sulfúrico é obtido como produto da reação do trióxido de enxofre com a água. A parte do ácido sulfúrico oriunda do trióxido de enxofre corresponde a $\frac{40}{49}$, já os $\frac{9}{49}$ restantes vêm da água. Sabendo disso um químico mistura 400 gramas de trióxido de enxofre com 135 gramas de água destilada. Após 5 minutos ele já possui 50 gramas de ácido sulfúrico. Quantos gramas de ácido sulfúrico ele terá após 20 minutos?

Primeiramente, temos que a equação que modela o problema é dada por

$$
\frac{d y}{d t}=k_{1}\left(400-\frac{40}{49} y\right)\left(135-\frac{9}{49} y\right)
$$

que corresponde a

$$
\frac{d y}{d t}=k_{1}\left(\frac{40}{49}\right)\left(\frac{9}{49}\right)(490-y)(735-y) .
$$


Ou seja,

$$
\frac{d y}{d t}=k(490-y)(735-y) .
$$

Então, segue de (3.24), $\operatorname{com} a=490$ e $b=735$, que

$$
y(t)=\frac{360150\left(e^{245 k t}-1\right)}{490 e^{245 k t}-735} .
$$

Agora, como $y(5)=50$, temos que

$$
50=\frac{360150\left(e^{1225 k}-1\right)}{490 e^{1225 k}-735} \Rightarrow 245 k \cong-0,0074
$$

Assim, a solução da equação que modela o problema é dada por

$$
y(t)=\frac{360150\left(e^{-0,0074 t}-1\right)}{490 e^{-0,0074 t}-735} .
$$

Portanto, para saber quantos gramas de ácido sulfúrico ele terá após 20 minutos, basta calcular

$$
y(20)=\frac{360150\left(e^{-0,148}-1\right)}{490 e^{-0,148}-735} \cong \frac{-49545,434}{-312,409} \cong 158,6 .
$$

Logo, após 20 minutos esse químico terá aproximadamente 158,6 gramas de ácido sulfúrico.

\subsubsection{Catenária}

A curva obtida quando um cabo, flexível e inextensível, é suspenso por suas extremidades e submetido apenas à ação do seu próprio peso é conhecida como catenária.

A primeira tentativa de resolver o problema de descrever matematicamente a forma da curva formada por um fio suspenso entre dois pontos e sob ação exclusiva da gravidade foi de Galileu Galilei, conjecturando que a curva fosse uma parábola. Entretanto, em 1647, Huyghens, aos 17 anos, mostrou que a conjectura era falsa, mas sem descobrir a expressão da curva. O problema foi resolvido somente em 1691 por Johann Bernoulli, Leibniz e Huyghens, individualmente. As três soluções consistiam em uma descrição geométrica da curva.

A fim de analisarmos este problema, consideremos um sistema de coordenadas com origem $O$ no ponto mais baixo da curva e o eixo $y$ coincidindo com a vertical. Sendo $P=(x, y)$ um ponto do cabo, consideremos o equilíbrio no trecho $O P$. Desse modo, $H+T+\omega s=0$, onde $H$ é a tensão do cabo em seu ponto mais baixo, $T$ é a tensão no ponto $P$ e $\omega s$ é o peso do trecho $O P$ do cabo, em que $\omega$ é o peso por unidade de comprimento e $s$ é o comprimento do arco $O P$. Sabemos que devido à flexibilidade do fio, a tensão $T$ age na direção tangente. A figura a seguir mostra a representação geométrica dessa situação. 
Figura 5 - Catenária

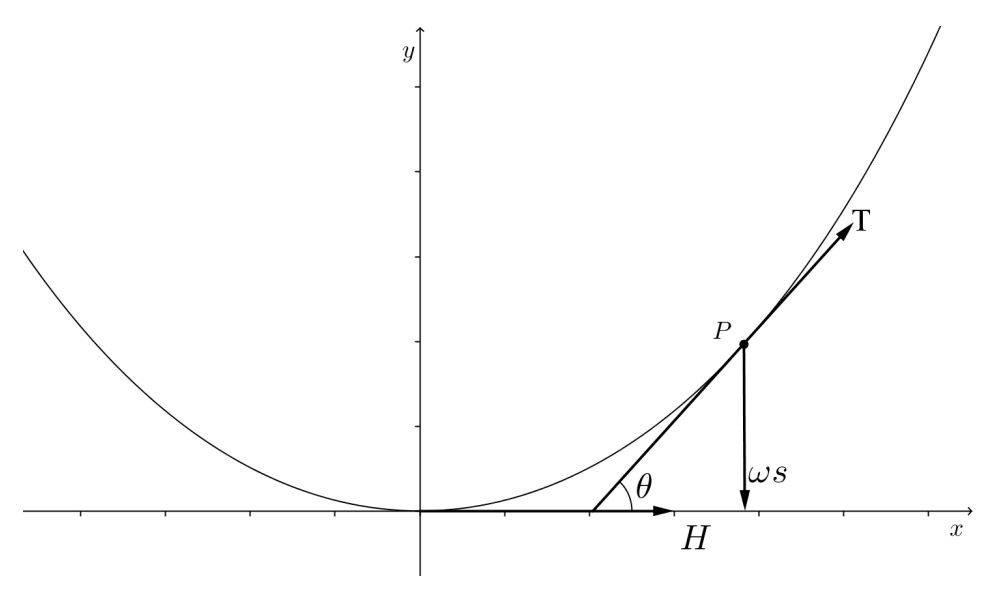

Fonte: Elaborada pelo autor.

Sendo $\theta$ o ângulo formado entre o eixo horizontal e a tensão, temos que

$$
T \cos (\theta)=H \quad \text { e } \quad T \operatorname{sen}(\theta)=\omega s .
$$

Segue daí que

$$
\tan (\theta)=\frac{\omega}{H} s
$$

Tomando $\frac{\omega}{H}=c$ e observando que $\tan (\theta)=y^{\prime}(x)$, pois $y(x)$ é a equação da curva, podemos escrever a equação acima como $y^{\prime}=c s$. Logo, derivando em relação a $x$ obtemos

$$
y^{\prime \prime}=c \frac{d s}{d x} .
$$

Claramente $s$ depende de $x$, basta ver que quanto maior for $x$ maior será $s$. Mais ainda, sabe-se que

$$
\frac{d s}{d x}=\sqrt{1+\left(\frac{d y}{d x}\right)^{2}} .
$$

Logo, fazendo a devida substituição obtemos

$$
y^{\prime \prime}=c \sqrt{1+\left(y^{\prime}\right)^{2}} .
$$

Para resolver a equação (3.25) podemos fazer a mudança de variável $p=y^{\prime}$. Desse modo, ficamos com

$$
p^{\prime}=c \sqrt{1+p^{2}}
$$

e esta é uma equação com variáveis separáveis. Então, podemos reescrevê-la na forma

$$
\frac{d p}{\sqrt{1+p^{2}}}=c d x
$$

Integrando ambos os membros obtemos

$$
\int \frac{d p}{\sqrt{1+p^{2}}}=\int c d x .
$$


Fazendo a substituição $p=\tan (\theta)$ e $d p=\sec ^{2}(\theta) d \theta \operatorname{com} \theta \in\left[0, \frac{\pi}{2}\right)$, vemos que

$$
\begin{aligned}
\int \frac{d p}{\sqrt{1+p^{2}}} & =\int \frac{\sec ^{2}(\theta)}{\sqrt{1+\tan ^{2}(\theta)}} d \theta \\
& =\int \frac{\sec ^{2}(\theta)}{\sec (\theta)} d \theta \\
& =\int \sec (\theta) d \theta=\ln \left(\tan \theta+\sqrt{1+\tan ^{2} \theta}\right) .
\end{aligned}
$$

Logo, a equação (3.26) fica da seguinte forma

$$
\ln \left(\tan \theta+\sqrt{1+\tan ^{2} \theta}\right)=c x+C_{1}, \quad \theta \in\left[0, \frac{\pi}{2}\right) .
$$

Reescrevendo a solução em função de $p$ temos

$$
\ln \left(p+\sqrt{1+p^{2}}\right)=c x+C_{1} .
$$

Uma vez que para $x=0$ temos $p(0)=y^{\prime}(0)=0$, segue da última equação que $C_{1}=0$. Assim,

$$
\ln \left(p+\sqrt{1+p^{2}}\right)=c x .
$$

Resolvendo a equação em $p$ temos

$$
\left(p+\sqrt{1+p^{2}}\right)=e^{c x}
$$

o que implica que

$$
p=\frac{e^{c x}-e^{-c x}}{2}
$$

Agora, como $p=\frac{d y}{d x}$ temos

$$
\frac{d y}{d x}=\frac{e^{c x}-e^{-c x}}{2}
$$

e integrando ambos os membros em relação a $x$, obtemos

$$
y(x)=\frac{e^{c x}+e^{-c x}}{2 c}+K .
$$

Uma vez que $y(0)=0$, obtemos $K=-c^{-1}$. Portanto,

$$
y(x)=\frac{e^{c x}+e^{-c x}}{2 c}-c^{-1} .
$$

Mais ainda, como a função cosseno hiperbólico é dada por $\cosh (a)=\frac{e^{a}+e^{-a}}{2}$ concluímos que

$$
y(x)=c^{-1}(\cosh (c x)-1)
$$

É interessante observar que a forma da catenária pode ser vista nos fios usados para o transporte de energia elétrica. Além disso, a catenária tem como característica o fato de que uma força aplicada em um ponto qualquer da curva é distribuída uniformemente por todo o material ao longo da curva e, por isso, ela é utilizada na fabricação de fundo de latas de refrigerante, túneis e iglus. 


\subsubsection{Corpo em Queda Livre com Influência do Atrito}

Como já foi visto, a variação da velocidade de um corpo em queda livre com influência do atrito pode ser estudada através da seguinte EDO

$$
m v^{\prime}(t)=m g-r_{a}
$$

onde $m$ é a massa do corpo, $g$ é a aceleração da gravidade e $r_{a}>0$ representa a resistência do ar. Uma vez que a resistência do ar pode depender, dentre outros fatores, da velocidade do corpo, a equação acima pode ser tratada como uma EDO com variáveis separáveis, como mostra o próximo exemplo.

Exemplo 34. Um paraquedista está submetido a uma velocidade de $105 \mathrm{~m} / \mathrm{s}$ no momento em que o paraquedas se abre. Sabendo que a resistência do ar é $\frac{P v^{2}}{25} \mathrm{~N}$, onde $P$ é o peso do paraquedista mais o paraquedas, medidos em newtons, e considerando $g=10 \mathrm{~m} / \mathrm{s}^{2}$, qual a velocidade do paraquedista depois de 3 segundos?

Notando que $m=\frac{P}{10}$ podemos modelar o problema dado através da EDO

$$
\frac{P}{10} v^{\prime}=\frac{P}{10} 10-\frac{P v^{2}}{25},
$$

que é equivalente a

$$
\frac{1}{10} \frac{d v}{d t}=\frac{25-v^{2}}{25}
$$

que é uma equação com variáveis separáveis. Então, podemos reescrevê-la como

$$
\frac{d v}{25-v^{2}}=\frac{10}{25} d t
$$

Integrando os dois membros da igualdade, e usando que $\int \frac{d u}{a^{2}-u^{2}}=\frac{1}{2 a} \ln \left|\frac{u+a}{u-a}\right|$, temos

$$
\frac{1}{10} \ln \left|\frac{v+5}{v-5}\right|=\frac{10}{25} t+c_{1}
$$

Logo,

$$
\ln \left|\frac{v+5}{v-5}\right|=4 t+c_{2}
$$

o que implica que

$$
\frac{v+5}{v-5}=e^{4 t} c
$$

Assim, a solução geral para a velocidade do corpo no instante $t$ é dada por

$$
v(t)=5 \frac{\left(e^{4 t} c+1\right)}{\left(e^{4 t} c-1\right)} .
$$

Aplicando agora a condição inicial $v(0)=105$, obtemos

$$
105=5 \frac{c+1}{c-1} \Rightarrow c=1,1 .
$$


Assim, concluímos que

$$
v(t)=5 \frac{\left(1,1 e^{4 t}+1\right)}{\left(1,1 e^{4 t}-1\right)} .
$$

Portanto, podemos ver que

$$
v(3)=5 \frac{\left(1,1 e^{12}+1\right)}{\left(1,1 e^{12}-1\right)} \cong 5 \frac{179031,2706}{179029,2706} \cong 5,
$$

ou seja, a velocidade do paraquedista depois de 3 segundos é de aproximadamente $5 \mathrm{~m} / \mathrm{s}$. 
CAPÍTULO

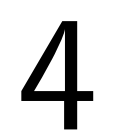

\section{EQUAÇÕES DIFERENCIAIS ORDINÁRIAS DE SEGUNDA ORDEM}

O objetivo deste capítulo é apresentar métodos de resolução para alguns tipos de equações diferenciais ordinárias de segunda ordem. Para isso, começamos lembrando que as EDOs de segunda ordem, geralmente, podem ser escritas da seguinte forma

$$
y^{\prime \prime}=f\left(t, y, y^{\prime}\right)
$$

onde $f$ é uma função definida em algum subconjunto aberto $A \subset R^{3}$.

Lembramos ainda que uma função $y=y(t)$ é uma solução da equação (4.1) num intervalo $I$, se possui segunda derivada em $I$ e satisfaz $y^{\prime \prime}(t)=f\left(t, y(t), y^{\prime}(t)\right)$ para todo $t \in I$.

Exemplo 35. As funções $y_{1}(t)=e^{2 t}$ e $y_{2}(t)=e^{-2 t}$ são soluções da equação $y^{\prime \prime}=4 y$, pois para todo $t \in \mathbb{R}$ temos que

$$
y_{1}(t)=e^{2 t} \Rightarrow y_{1}^{\prime}(t)=2 e^{2 t} \Rightarrow y_{1}^{\prime \prime}(t)=4 e^{2 t}=4 y_{1}(t)
$$

e

$$
y_{2}(t)=e^{-2 t} \Rightarrow y_{2}^{\prime}(t)=-2 e^{-2 t} \Rightarrow y_{2}^{\prime \prime}(t)=4 e^{-2 t}=4 y_{2}(t)
$$

Agora, para motivar o conceito de um P.V.I. para uma equação de segunda ordem, consideremos a equação $y^{\prime \prime}=3$. Para encontrarmos uma solução desta equação basta integrarmos duas vezes. De fato,

$$
y^{\prime \prime}(t)=3 \Rightarrow y^{\prime}(t)=\int 3 d t=3 t+c_{1} \Rightarrow y(t)=\int\left(3 t+c_{1}\right) d t=\frac{3 t^{2}}{2}+c_{1} t+c_{2} .
$$

Observe que agora a solução geral apresenta duas constantes arbitrárias, $c_{1}$ e $c_{2}$. Mas note que se, por exemplo, $y^{\prime}(0)=1$ teremos

$$
1=y^{\prime}(0)=3 \cdot 0+c_{1}
$$


o que implica que $c_{1}=1 \mathrm{e}$, assim,

$$
y(t)=\frac{3 t^{2}}{2}+t+c_{2} .
$$

Além disso, se, por exemplo, $y(0)=2$ teremos

$$
2=y(0)=c_{2},
$$

ou seja, $c_{2}=2$ e, então,

$$
y(t)=\frac{3 t^{2}}{2}+t+2
$$

será a solução do problema

$$
\left\{\begin{array}{l}
y^{\prime \prime}=3 \\
y(0)=2 \\
y^{\prime}(0)=1 .
\end{array}\right.
$$

Assim, podemos perceber que, para conseguirmos a unicidade de solução, precisamos de duas condições, uma sobre $y(t)$ e outra sobre $y^{\prime}(t)$ num instante $t_{0}$. Desse modo, um problema de valor inicial para a equação (4.1) é dado por

$$
\left\{\begin{array}{l}
y^{\prime \prime}=f\left(t, y, y^{\prime}\right), \\
y\left(t_{0}\right)=y_{0} \\
y^{\prime}\left(t_{0}\right)=z_{0}
\end{array}\right.
$$

Agora, veja que para estudarmos métodos de resolução para a equação (4.1), precisamos, inicialmente, ter informações sobre a existência de soluções para esta equação. No entanto, em geral, é difícil resolver a equação (4.1), e por esse motivo, é usual recorrer a equações mais simples, como as lineares que são modelos aproximados de muitas equações diferenciais não lineares. Portanto, nossa atenção agora será voltada para as equações lineares, introduzidas a seguir.

\subsection{Equações Diferenciais Lineares de Segunda Ordem}

Definição 18. Uma equação diferencial linear de segunda ordem é uma equação da forma

$$
y^{\prime \prime}+a(t) y^{\prime}+b(t) y=g(t), \quad[\text { L.N.H. }]
$$

onde $a(t), b(t)$ e $g(t)$ são funções contínuas num intervalo $I$ da reta. Esta equação é chamada de linear não homogênea [L.N.H.]. Quando $g(t) \equiv 0$ ela torna-se

$$
y^{\prime \prime}+a(t) y^{\prime}+b(t) y=0, \quad[\text { L.H. }]
$$

e é chamada de linear homogênea [L.H.]. 
Como estamos interessados em encontrar soluções para equações lineares de segunda ordem, apresentamos a seguir o Teorema de Existência e Unicidade para este caso particular.

Teorema 3. (Existência e Unicidade) Se as funções $a(t), b(t)$ e $g(t)$ são contínuas num intervalo $I$, então dados $t_{0} \in I$ e $y_{0}, z_{0} \in \mathbb{R}$, o P.V.I.

$$
\left\{\begin{array}{l}
y^{\prime \prime}+a(t) y^{\prime}+b(t) y=g(t) \\
y\left(t_{0}\right)=y_{0} \\
y^{\prime}\left(t_{0}\right)=z_{0}
\end{array}\right.
$$

possui uma única solução $y=y(t)$, no intervalo $I$.

Demonstração. Do Teorema de Existência e Unicidade para EDOs de primeira ordem, sabemos que se $F$ e $\frac{\partial F}{\partial x}$ são função contínuas, então o P.V.I.

$$
\left\{\begin{array}{l}
x^{\prime}=F(t, x) \\
x\left(t_{0}\right)=x_{0}
\end{array}\right.
$$

possui uma única solução em $I$. Agora, note que fazendo $y=x_{1}$ e $y^{\prime}=x_{2}$, a equação

$$
y^{\prime \prime}+a(t) y^{\prime}+b(t) y=g(t)
$$

pode ser escrita na forma $x^{\prime}=F(t, x)$, pois como $x_{1}^{\prime}=y^{\prime}=x_{2}$ e $x_{2}^{\prime}=y^{\prime \prime}=-a(t) x_{2}-b(t) x_{1}+g(t)$, chamando $x=\left(\begin{array}{l}x_{1} \\ x_{2}\end{array}\right)$, temos que

$$
x^{\prime}=\left(\begin{array}{c}
x_{1}^{\prime} \\
x_{2}^{\prime}
\end{array}\right)=\left(\begin{array}{c}
x_{2} \\
-a(t) x_{2}-b(t) x_{1}+g(t)
\end{array}\right)=\left(\begin{array}{l}
F_{1}(t, x) \\
F_{2}(t, x)
\end{array}\right)=F(t, x) .
$$

Neste caso, $\frac{\partial F}{\partial x}$ representa a matriz jacobiana de $F\left(t, x_{1}, x_{2}\right)$ em relação a $x_{1}, x_{2}$, isto é,

$$
J F\left(t, x_{1}, x_{2}\right)=\frac{\partial\left(F_{1}, F_{2}\right)}{\partial\left(x_{1}, x_{2}\right)}=\left(\begin{array}{ll}
\frac{\partial F_{1}}{\partial x_{1}} & \frac{\partial F_{1}}{\partial x_{2}} \\
\frac{\partial F_{2}}{\partial x_{1}} & \frac{\partial F_{2}}{\partial x_{2}}
\end{array}\right)=\left(\begin{array}{cc}
0 & 1 \\
-b(t) & -a(t)
\end{array}\right) .
$$

Logo, como $a(t), b(t)$ e $g(t)$ são funções contínuas em $I$, temos que $F$ e $\frac{\partial F}{\partial x}$ são contínuas em uma vizinhança de $\left(t_{0}, x_{0}\right)$ e o P.V.I.

$$
\left\{\begin{array}{l}
x^{\prime}=F(t, x) \\
x\left(t_{0}\right)=x_{0}
\end{array}\right.
$$

possui uma única solução em $I$. Ou seja, o P.V.I.

$$
\left\{\begin{array}{l}
y^{\prime \prime}+a(t) y^{\prime}+b(t) y=g(t) \\
y\left(t_{0}\right)=y_{0} \\
y^{\prime}\left(t_{0}\right)=z_{0}
\end{array}\right.
$$

possui uma única solução em $I$. 
Observação 22. Note que, pelo teorema anterior, a única solução da equação [L.H.] tal que $y\left(t_{0}\right)=y^{\prime}\left(t_{0}\right)=0$ é a função $y(t) \equiv 0$.

\subsubsection{Equações Lineares Homogêneas}

Nesta seção, veremos alguns métodos de resolução para a equação linear homogênea. O resultado a seguir é fundamental no estudo que faremos para encontrar o conjunto de soluções da equação diferencial [L.H.].

Teorema 4. (Princípio da Superposição) Se $\varphi_{1}(t)$ e $\varphi_{2}(t)$ são soluções de [L.H.] e $c_{1}$ e $c_{2}$ são constantes reais, então a função $\varphi(t)=c_{1} \varphi_{1}(t)+c_{2} \varphi_{2}(t)$ também é solução de [L.H.].

Demonstração. Substituindo $\varphi(t)$ em [L.H.], temos

$$
\begin{aligned}
y^{\prime \prime}+a(t) y^{\prime}+b(t) y & =\varphi^{\prime \prime}(t)+a(t) \varphi^{\prime}(t)+b(t) \varphi(t) \\
& =c_{1}\left[\varphi_{1}^{\prime \prime}(t)+a(t) \varphi_{1}^{\prime}(t)+b(t) \varphi_{1}(t)\right]+c_{2}\left[\varphi_{2}^{\prime \prime}(t)+a(t) \varphi_{2}^{\prime}(t)+b(t) \varphi_{2}(t)\right] \\
& =0
\end{aligned}
$$

pois $\varphi_{1}(t)$ e $\varphi_{2}(t)$ são soluções de [L.H.]. Logo, $\varphi(t)$ é solução de [L.H.].

Observação 23. Do Teorema 4, podemos concluir que o conjunto das soluções de [L.H.] é um espaço vetorial de dimensão 2 e veremos que, sob determinadas condições, as funções $\varphi_{1}$ e $\varphi_{2}$ formam uma base para esse espaço.

Seja $y(t)$ uma solução de [L.H.] e sejam $y_{0}=y\left(t_{0}\right), z_{0}=y^{\prime}\left(t_{0}\right)$ com $t_{0} \in I$ fixado. Para que $y(t)$ seja dada por $y(t)=c_{1} y_{1}(t)+c_{2} y_{2}(t)$, onde $y_{1}$ e $y_{2}$ são soluções da [L.H.] devemos ter

$$
\left\{\begin{array}{l}
c_{1} y_{1}\left(t_{0}\right)+c_{2} y_{2}\left(t_{0}\right)=y_{0} \\
c_{1} y_{1}^{\prime}\left(t_{0}\right)+c_{2} y_{2}^{\prime}\left(t_{0}\right)=z_{0},
\end{array}\right.
$$

que podemos considerar como um sistema de duas equações nas incógnitas $c_{1}$ e $c_{2}$. Para que este sistema tenha solução única é necessário e suficiente que

$$
D=\left|\begin{array}{ll}
y_{1}\left(t_{0}\right) & y_{2}\left(t_{0}\right) \\
y_{1}^{\prime}\left(t_{0}\right) & y_{2}^{\prime}\left(t_{0}\right)
\end{array}\right| \neq 0,
$$

o que nos resulta no seguinte teorema.

Teorema 5. Sejam $y_{1}(t)$ e $y_{2}(t)$ soluções de [L.H.] tais que

$$
\left|\begin{array}{ll}
y_{1}(t) & y_{2}(t) \\
y_{1}^{\prime}(t) & y_{2}^{\prime}(t)
\end{array}\right| \neq 0
$$

para todo $t \in I$. Então, toda solução de [L.H.] é dada por $y(t)=c_{1} y_{1}(t)+c_{2} y_{2}(t)$. 
Definição 19. Nas condições do Teorema $5, y(t)=c_{1} y_{1}(t)+c_{2} y_{2}(t)$ é chamada de solução geral de [L.H.], ou $y_{1}(t)$ e $y_{2}(t)$ são soluções linearmente independentes de [L.H.].

Definição 20. O determinante (4.2) é chamado de Wronskiano de $y_{1}(t)$ e $y_{2}(t)$ e será denotado por $W\left[y_{1}, y_{2}\right](t)$ ou $W(t)$.

Veremos que o Wronskiano desempenha um papel importante no estudo de [L.H.].

Observação 24. Note que o Teorema 5 reduz o problema de encontrar a solução geral de [L.H.] ao problema de encontrar duas soluções "convenientes" $y_{1}(t)$ e $y_{2}(t)$ (isto é, tais que para todo $t \in I, W\left[y_{1}, y_{2}\right](t) \neq 0$, ou seja, $y_{1}(t)$ e $y_{2}(t)$ são linearmente independentes).

Observação 25. Se $W\left[y_{1}, y_{2}\right](t) \equiv 0$, podem existir soluções de [L.H.] que não são dadas por $y(t)=c_{1} y_{1}(t)+c_{2} y_{2}(t)$.

Exemplo 36. Consideremos a equação $y^{\prime \prime}-4 y^{\prime}-12 y=0$ e tomemos $y_{1}(t)=e^{6 t}$ e $y_{2}(t)=3 e^{6 t}$. Temos que $y_{1}(t)$ e $y_{2}(t)$ são soluções de [L.H.] e é fácil verificar que $W\left[y_{1}, y_{2}\right](t) \equiv 0$. Agora, note que $y_{3}(t)=e^{-2 t}$ também é solução da equação, mas não pode ser escrita como $c_{1} e^{6 t}+3 c_{2} e^{6 t}$.

Teorema 6. Sejam $y_{1}(t)$ e $y_{2}(t)$ soluções de [L.H.] em $I$ e $t_{0} \in I$ fixado. Seja $W(t)$ o Wronskiano de $y_{1}$ e $y_{2}$. Então,

$$
W(t)=W\left(t_{0}\right) e^{-\int_{t_{0}}^{t} a(s) d s}, \quad t \in I .
$$

Em particular, como a função exponencial nunca se anula, segue que se $W\left(t_{0}\right) \neq 0$, então $W(t) \neq 0$ para todo $t \in I$.

Demonstração. Como $W(t)=y_{1}(t) y_{2}^{\prime}(t)-y_{1}^{\prime}(t) y_{2}(t)$, então

$$
\begin{aligned}
W^{\prime}(t) & =y_{1}^{\prime}(t) y_{2}^{\prime}(t)+y_{1}(t) y_{2}^{\prime \prime}(t)-y_{1}^{\prime \prime}(t) y_{2}(t)-y_{1}^{\prime}(t) y_{2}^{\prime}(t) \\
& =y_{1}(t)\left[-a(t) y_{2}^{\prime}(t)-b(t) y_{2}(t)\right]-y_{2}(t)\left[-a(t) y_{1}^{\prime}(t)-b(t) y_{1}(t)\right] \\
& =-a(t)\left[y_{1}(t) y_{2}^{\prime}(t)-y_{1}^{\prime}(t) y_{2}(t)\right] \\
& =-a(t) W(t) .
\end{aligned}
$$

Ou seja, temos que $W^{\prime}(t)+a(t) W(t)=0$, que é uma EDO linear de primeira ordem em $W$, cuja solução geral é dada por

$$
W(t)=c e^{-\int_{t_{0}}^{t} a(s) d s} .
$$

No entanto, conhecendo o valor de $W\left(t_{0}\right)$, da equação (4.3) temos que $c=W\left(t_{0}\right)$ e, portanto,

$$
W(t)=W\left(t_{0}\right) e^{-\int_{t_{0}}^{t} a(s) d s}, \quad t \in I .
$$

Observação 26. Note que as conclusões do Teorema 6 referem-se apenas ao intervalo $I$ onde as funções $a(t)$ e $b(t)$ são contínuas. 
Finalmente, notamos que é sempre possível obter duas soluções $y_{1}$ e $y_{2}$ de [L.H.] tais que $W\left[y_{1}, y_{2}\right](t) \neq 0$ para todo $t \in I$, pois fixado $t_{0} \in I$, basta definir $y_{1}(t)$ como sendo a única solução de [L.H.] tal que $y\left(t_{0}\right)=1$ e $y^{\prime}\left(t_{0}\right)=0$ e $y_{2}$ como sendo a única solução de [L.H.] tal que $y\left(t_{0}\right)=0$ e $y^{\prime}\left(t_{0}\right)=1$. Assim, $W\left(t_{0}\right)=1$ e segue do Teorema 6 que $W(t) \neq 0$ para todo $t \in I$. Dado isto podemos concluir o seguinte teorema.

Teorema 7. Suponhamos que $a(t)$ e $b(t)$ sejam funções contínuas no intervalo $I$. Então, existem duas soluções $y_{1}(t)$ e $y_{2}(t)$ da equação [L.H.] tais que $W\left[y_{1}, y_{2}\right](t) \neq 0$, para todo $t \in I$. Além disso, a solução geral desta equação é dada por $c_{1} y_{1}(t)+c_{2} y_{2}(t)$, onde $c_{1}$ e $c_{2}$ são constantes arbitrárias.

\subsubsection{Método da Redução de Ordem}

Suponha conhecida uma solução não nula $y_{1}(t)$ de [L.H.]. Sabemos que, para toda constante $c \in \mathbb{R}, c y_{1}(t)$ também é solução de [L.H.]. Aqui temos uma motivação para tentarmos encontrar uma outra solução de [L.H.] da forma $y_{2}(t)=v(t) y_{1}(t)$, sendo $v(t)$ uma função não constante. Devido a D'Alembert (1717 - 1783), esse método é usualmente chamado de método da redução de ordem. Agora, note que

$$
y_{2}=v y_{1} \Rightarrow y_{2}^{\prime}=v^{\prime} y_{1}+v y_{1}^{\prime} \Rightarrow y_{2}^{\prime \prime}=v^{\prime \prime} y_{1}+2 v^{\prime} y_{1}^{\prime}+v y_{1}^{\prime \prime} \text {. }
$$

Substituindo em [L.H.], temos

$$
v^{\prime \prime} y_{1}+2 v^{\prime} y_{1}^{\prime}+v y_{1}^{\prime \prime}+a(t)\left[v^{\prime} y_{1}+v y_{1}^{\prime}\right]+b(t)\left[v y_{1}\right]=0,
$$

e, organizando de modo adequado, obtemos

$$
v^{\prime \prime} y_{1}+v^{\prime}\left[2 y_{1}^{\prime}+a(t) y_{1}\right]+v\left[y_{1}^{\prime \prime}+a(t) y_{1}^{\prime}+b(t) y_{1}\right]=0 \text {. }
$$

Como $y_{1}$ é solução de [L.H.], ficamos com

$$
v^{\prime \prime}+\left(a(t)+\frac{2 y_{1}^{\prime}}{y_{1}}\right) v^{\prime}=0 .
$$

Portanto, devemos encontrar a solução desta equação para encontrar $v(t)$ e, portanto, $y_{2}(t)$. Para isso, fazemos a mudança $z=v^{\prime}$ e ficamos com a seguinte EDO de primeira ordem

$$
z^{\prime}+\left(a(t)+\frac{2 y_{1}^{\prime}}{y_{1}}\right) z=0
$$

cuja solução é dada por

$$
z(t)=c e^{-\int\left[a(t)+2\left(\frac{y_{1}^{\prime}(t)}{y_{1}(t)}\right)\right] d t}:=c g(t),
$$

onde $c$ é uma constante. Logo,

$$
v(t)=\int z(t) d t=c \int g(t) d t
$$


Tomando $c=1$, temos que $v(t)=\int g(t) d t$ é uma função não constante e

$$
\begin{aligned}
y_{2}(t) & =v(t) y_{1}(t) \\
& =y_{1}(t) \int g(t) d t
\end{aligned}
$$

é uma solução de [L.H.].

É fácil verificar que $y_{1}(t)$ e $y_{2}(t)$ são linearmente independentes num intervalo $I$ tal que $y_{1}(t) \neq 0$, para todo $t \in I$. De fato,

$$
\begin{aligned}
W(t) & =\left|\begin{array}{ll}
y_{1}(t) & y_{2}(t) \\
y_{1}^{\prime}(t) & y_{2}^{\prime}(t)
\end{array}\right| \\
& =y_{1}(t) y_{2}^{\prime}(t)-y_{1}^{\prime}(t) y_{2}(t) \\
& =y_{1}(t)\left(v^{\prime}(t) y_{1}(t)+v(t) y_{1}^{\prime}(t)\right)-y_{1}^{\prime}(t) v(t) y_{1}(t) \\
& =v^{\prime}(t) y_{1}^{2}(t) \\
& =z(t) y_{1}^{2}(t) \\
& =g(t) y_{1}^{2}(t) \neq 0,
\end{aligned}
$$

para todo $t \in I$.

Note que acima poderíamos ter tomado qualquer $c \neq 0$.

Exemplo 37. Encontremos a segunda solução de $y^{\prime \prime}-2 y^{\prime}+y=0$ sabendo que $y_{1}(t)=e^{t}$ é uma solução desta equação.

Vamos procurar $y_{2}(t)=v(t) y_{1}(t)=v e^{t}$. Observe que $y_{2}^{\prime}=v^{\prime} e^{t}+v e^{t}$ e também que $y_{2}^{\prime \prime}=v^{\prime \prime} e^{t}+2 v^{\prime} e^{t}+v e^{t}$. Logo, substituindo na EDO obtemos

$$
\left(v^{\prime \prime} e^{t}+2 v^{\prime} e^{t}+v e^{t}\right)-2\left(v^{\prime} e^{t}+v e^{t}\right)+\left(v e^{t}\right)=0 .
$$

Simplificando, ficamos com

$$
v^{\prime \prime} e^{t}=0
$$

o que implica que

$$
v(t)=c t .
$$

Como desejamos apenas uma das possíveis funções $y_{2}(t)$, com $v(t)$ não contante, podemos tomar $c=1 \mathrm{e}$, assim,

$$
y_{2}(t)=t e^{t}
$$

Exemplo 38. Encontremos a segunda solução de $t^{2} y^{\prime \prime}+2 t y^{\prime}-2 y=0$ sabendo que $y_{1}(t)=t$ é uma solução desta equação.

Vamos procurar $y_{2}(t)=v(t) y_{1}(t)=v t$. Observando que $y_{2}^{\prime}=v^{\prime} t+v$ e também que $y_{2}^{\prime \prime}=v^{\prime \prime} t+2 v^{\prime}$ e substituindo na EDO obtemos

$$
t^{2}\left(v^{\prime \prime} t+2 v^{\prime}\right)+2 t\left(v^{\prime} t+v\right)-2 v t=0 .
$$


Simplificando, ficamos com

$$
t^{3} v^{\prime \prime}+4 t^{2} v^{\prime}=0
$$

e fazendo $z=v^{\prime}$ obtemos a seguinte EDO em $z$

$$
t^{3} z^{\prime}+4 t^{2} z=0 \Rightarrow z^{\prime}+\frac{4}{t} z=0
$$

que é uma equação linear homogênea de primeira ordem, cuja solução é $z(t)=c t^{-4}$. Então, concluímos que

$$
v(t)=\int c t^{-4} d t=c\left(-\frac{1}{3} t^{-3}+c_{1}\right) .
$$

Como desejamos apenas uma das possíveis funções $y_{2}(t)$ com $v(t)$ não constante, podemos tomar $c=1$ e $c_{1}=0$, assim,

$$
y_{2}(t)=-\frac{1}{3} t^{-2}
$$

\subsubsection{Equações Homogêneas com Coeficientes Constantes}

Consideremos a equação

$$
a y^{\prime \prime}+b y^{\prime}+c y=0,
$$

onde $a, b$ e $c$ são constantes reais $\operatorname{com} a \neq 0$.

Nosso objetivo é encontrar duas soluções linearmente independentes para esta equação, pois como já vimos, todas as demais serão combinações dessas.

Note que, se $y(t)=\varphi(t)$ é uma solução da equação (4.4), então a soma dos termos $a \varphi^{\prime \prime}(t), b \varphi^{\prime}(t)$ e $c \varphi(t)$ deve ser igual a zero para todo $t$. Para que isto ocorra as três funções $\varphi(t)$, $\varphi^{\prime}(t)$ e $\varphi^{\prime \prime}(t)$, devem ser do "mesmo tipo". Por exemplo, a função $y(t)=t^{4}$ nunca será solução de (4.4), pois os termos $12 a t^{2}, 4 b t^{3}$ e $c t^{4}$ são polinômios de graus diferentes e sua soma não se cancela. Já a função $y(t)=e^{\lambda t}$, onde $\lambda$ é constante, tem a propriedade de que tanto $y^{\prime}$, quanto $y^{\prime \prime}$ são múltiplos de $y(t)$. Isto sugere tentarmos $y(t)=e^{\lambda t}$ como solução de (4.4). Substituindo na equação temos

$$
a\left(e^{\lambda t}\right)^{\prime \prime}+b\left(e^{\lambda t}\right)^{\prime}+c e^{\lambda t}=0 \Rightarrow e^{\lambda t}\left(a \lambda^{2}+b \lambda+c\right)=0
$$

o que implica que

$$
a \lambda^{2}+b \lambda+c=0
$$

Portanto, $y(t)=e^{\lambda t}$ é uma solução de (4.4) se, e somente se, $\lambda$ é raiz de (4.5).

Definição 21. A equação (4.5) é chamada equação característica de (4.4).

Sabemos que as raízes da equação característica são

$$
\lambda_{1}=\frac{-b+\sqrt{b^{2}-4 a c}}{2 a} \quad \text { e } \quad \lambda_{2}=\frac{-b-\sqrt{b^{2}-4 a c}}{2 a} .
$$

A seguir, analisaremos as três possibilidades para o discriminante $b^{2}-4 a c$. 


\section{i $b^{2}-4 a c>0$ - Raízes reais distintas}

Neste caso, $\lambda_{1} \neq \lambda_{2}$ e, desse modo, $y_{1}(t)=e^{\lambda_{1} t}$ e $y_{2}(t)=e^{\lambda_{2} t}$ são duas soluções distintas de (4.4). Além disso,

$$
W(t)=\left|\begin{array}{cc}
e^{\lambda_{1} t} & e^{\lambda_{2} t} \\
\lambda_{1} e^{\lambda_{1} t} & \lambda_{2} e^{\lambda_{2} t}
\end{array}\right|=\left(\lambda_{2}-\lambda_{1}\right) e^{\left(\lambda_{1}+\lambda_{2}\right) t} \neq 0
$$

para todo $t \in \mathbb{R}$. Logo, as soluções são linearmente independentes e formam uma base para o espaço das soluções, isto é, qualquer solução é da forma

$$
y(t)=c_{1} e^{\lambda_{1} t}+c_{2} e^{\lambda_{2} t} .
$$

Exemplo 39. Encontremos a solução geral de $y^{\prime \prime}-5 y^{\prime}+6 y=0$.

Para isso, note que a equação característica para esta EDO é

$$
\lambda^{2}-5 \lambda+6=0
$$

que tem como raízes $\lambda_{1}=3$ e $\lambda_{2}=2$. Como $\lambda_{1} \neq \lambda_{2}$, a solução geral da EDO é

$$
y(t)=c_{1} e^{3 t}+c_{2} e^{2 t}
$$

ii $b^{2}-4 a c=0$ - Raízes reais iguais

Neste caso, $\lambda_{1}=\lambda_{2}=-\frac{b}{2 a}$ e, desse modo, temos apenas uma solução $y_{1}(t)=e^{\left(-\frac{b}{2 a}\right) t}$. Para encontrar outra solução, que não seja múltipla de $y_{1}$, usaremos o método da redução de ordem, isto é, procuraremos $v(t)$ não constante tal que $y_{2}(t)=v(t) e^{\left(-\frac{b}{2 a}\right) t}$ seja solução de (4.4). Fazendo a substituição em (4.4), ficamos com

$$
e^{\left(-\frac{b}{2 a}\right) t}\left[a v^{\prime \prime}+\left(\frac{b^{2}}{4 a}-\frac{b^{2}}{2 a}+c\right) v\right]=0 .
$$

Como $e^{\left(-\frac{b}{2 a}\right) t}$ não se anula, devemos ter necessariamente que

$$
\left[a v^{\prime \prime}+\left(\frac{b^{2}}{4 a}-\frac{b^{2}}{2 a}+c\right) v\right]=0 \Rightarrow a v^{\prime \prime}=\left(-\frac{b^{2}}{4 a}+\frac{b^{2}}{2 a}-c\right) v=\left(\frac{b^{2}-4 a c}{4 a}\right) v
$$

Como $b^{2}-4 a c=0$ concluímos que

$$
a v^{\prime \prime}=0 \Rightarrow v^{\prime \prime}=0 \Rightarrow v^{\prime}=\alpha \Rightarrow v=\alpha t+\beta, \quad \alpha, \beta, \in \mathbb{R}
$$

Uma vez que queremos apenas uma das soluções, podemos tomar $\alpha=1$ e $\beta=0$. Sendo assim, $v(t)=t$ e a segunda solução de (4.4) é

$$
y_{2}(t)=t e^{\left(-\frac{b}{2 a}\right) t} .
$$


Exemplo 40. Encontremos a solução do P.V.I.

$$
\left\{\begin{array}{l}
y^{\prime \prime}+8 y^{\prime}+16 y=0, \\
y(0)=1, \\
y^{\prime}(0)=2 .
\end{array}\right.
$$

A equação característica dessa EDO é

$$
\lambda^{2}+8 \lambda+16=0
$$

que tem raízes reais iguais $\lambda_{1}=\lambda_{2}=-4$. Assim, uma das soluções é $y_{1}(t)=e^{-4 t}$ e já vimos, pelo método da redução de ordem, que a outra solução é $y_{2}(t)=t e^{-4 t}$. Portanto, a solução geral da EDO é dada por

$$
y(t)=c_{1} e^{-4 t}+c_{2} t e^{-4 t},
$$

o que implica que $y^{\prime}(t)=e^{-4 t}\left(-4 c_{1}+c_{2}-4 c_{2} t\right)$. Aplicando a primeira condição temos que

$$
1=y(0)=c_{1} \Rightarrow c_{1}=1,
$$

e, aplicando a segunda condição, obtemos

$$
y^{\prime}(0)=1\left(-4+c_{2}-4 c_{2} 0\right) \Rightarrow 2=c_{2}-4 \Rightarrow c_{2}=6
$$

Portanto, a solução do P.V.I. é dada por

$$
y(t)=e^{-4 t}+6 t e^{-4 t}
$$

iii $b^{2}-4 a c<0$ - Raízes complexas

Neste caso,

$$
\lambda_{1}=-\frac{b}{2 a}+\frac{i \sqrt{4 a c-b^{2}}}{2 a} \quad \text { e } \quad \lambda_{2}=-\frac{b}{2 a}-\frac{i \sqrt{4 a c-b^{2}}}{2 a} .
$$

Gostaríamos que $e^{\lambda_{1} t}$ e $e^{\lambda_{2} t}$ fossem soluções de (4.4). Contudo, devemos resolver primeiro dois problemas que aparecem:

1) definir $e^{\lambda t}$ para $\lambda$ complexo;

2) mesmo definindo $e^{\lambda_{1} t}$ e $e^{\lambda_{2} t}$ como soluções (com valores complexos), queremos soluções reais.

Inicialmente, voltemos nossa atenção para o segundo problema, pois sem ele o primeiro não teria sentido. Para isso, consideremos a seguinte definição. 
Definição 22. Se $F(t)=u(t)+i v(t)$, definimos $F^{\prime}(t)=u^{\prime}(t)+i v^{\prime}(t)$.

Observação 27. A definição acima faz sentido, pois podemos identificar $F(t)=u(t)+i v(t)$ como $f(t)=(u(t), v(t))$. Logo, $f(t)$ é uma parametrização de uma curva plana cujo vetor velocidade é $\left(u^{\prime}(t), v^{\prime}(t)\right)$. Desse modo, é natural a definição dada.

Proposição 6. Se $y(t)=u(t)+i v(t)$ é uma solução complexa de (4.4), então $u(t)$ e $v(t)$ são soluções reais de (4.4).

Demonstração. Substituindo $y(t)=u(t)+i v(t)$ em (4.4) obtemos

$$
\begin{aligned}
a y^{\prime \prime}(t)+b y^{\prime}(t)+c y=0 & \Rightarrow a(u(t)+i v(t))^{\prime \prime}+b(u(t)+i v(t))^{\prime}+c(u(t)+i v(t))=0 \\
& \Rightarrow\left[a u^{\prime \prime}(t)+b u^{\prime}(t)+c u(t)\right]+i\left[a v^{\prime \prime}(t)+b v^{\prime}(t)+c v(t)\right]=0 .
\end{aligned}
$$

Agora, para que um número complexo seja zero é necessário que tanto sua parte real quanto sua parte imaginária sejam zero, ou seja,

$$
a u^{\prime \prime}(t)+b u^{\prime}(t)+c u(t)=0 \quad \text { e } \quad a v^{\prime \prime}(t)+b v^{\prime}(t)+c v(t)=0 .
$$

Logo, $u(t)$ e $v(t)$ são soluções reais de (4.4).

Agora, com o segundo problema resolvido, podemos passar para o primeiro problema, ou seja, definir $e^{\lambda t}$ para $\lambda$ complexo. Para isso, veja que é natural pedir que a função satisfaça a propriedade $e^{a+b}=e^{a} e^{b}$. Logo, se $\lambda=\alpha+i \beta$ devemos ter

$$
e^{\lambda t}=e^{\alpha t+i \beta t}=e^{\alpha t} e^{i \beta t}
$$

Desse modo, basta definir $e^{i \beta t}$. Agora, sabemos que para todo $x$ real vale

$$
e^{x}=\sum_{n=0}^{\infty} \frac{x^{n}}{n !}=1+x+\frac{x^{2}}{2 !}+\frac{x^{3}}{3 !}+\cdots,
$$

e esta equação também tem sentido para $x$ complexo. Isto nos leva a colocar

$$
\begin{aligned}
e^{i \theta} & =1+i \theta+\frac{(i \theta)^{2}}{2 !}+\frac{(i \theta)^{3}}{3 !}+\cdots \\
& =1+i \theta-\frac{\theta^{2}}{2 !}-\frac{i \theta^{3}}{3 !}+\frac{\theta^{4}}{4 !}+\cdots \\
& =\left(1-\frac{\theta^{2}}{2 !}+\frac{\theta^{4}}{4 !}-\cdots\right)+i\left(\theta-\frac{\theta^{3}}{3 !}+\frac{\theta^{5}}{5 !}-\cdots\right) .
\end{aligned}
$$

Mas é bem conhecido que $\cos \theta=1-\frac{\theta^{2}}{2 !}+\frac{\theta^{4}}{4 !}-\cdots$ e $\operatorname{sen} \theta=\theta-\frac{\theta^{3}}{3 !}+\frac{\theta^{5}}{5 !}-\cdots$. Logo, é natural definirmos

$$
e^{i \theta}=\cos \theta+i \operatorname{sen} \theta
$$

Portanto,

$$
e^{\lambda t}=e^{(\alpha+i \beta) t}=e^{\alpha t}(\cos \beta t+i \operatorname{sen} \beta t) .
$$


Lema 3. Se $\lambda$ é um número complexo, então $\frac{d e^{\lambda t}}{d t}=\lambda e^{\lambda t}$.

Demonstração. Seja $\lambda=\alpha+i \beta, \alpha, \beta \in \mathbb{R}$. Então,

$$
\begin{aligned}
\frac{d e^{\lambda t}}{d t}=\frac{d e^{(\alpha+i \beta) t}}{d t} & =\frac{d}{d t}\left(e^{\alpha t} e^{i \beta t}\right) \\
& =\frac{d}{d t}\left[e^{\alpha t}(\cos \beta t+i \operatorname{sen} \beta t)\right] \\
& =\frac{d}{d t}\left(e^{\alpha t} \cos \beta t\right)+i \frac{d}{d t}\left(e^{\alpha t} \operatorname{sen} \beta t\right) \\
& =\left(\alpha e^{\alpha t} \cos \beta t-\beta e^{\alpha t} \operatorname{sen} \beta t\right)+i\left(\alpha e^{\alpha t} \operatorname{sen} \beta t+\beta e^{\alpha t} \cos \beta t\right) \\
& =\alpha e^{\alpha t}(\cos \beta t+i \operatorname{sen} \beta t)+\beta e^{\alpha t} i(\cos \beta t+i \operatorname{sen} \beta t) \\
& =\alpha e^{(\alpha+i \beta) t}+i \beta e^{(\alpha+i \beta) t} \\
& =(\alpha+i \beta) e^{(\alpha+i \beta) t}=\lambda e^{\lambda t}
\end{aligned}
$$

Agora, fica fácil verificar que

$$
y(t)=e^{\lambda t}=e^{\alpha t}(\cos \beta t+i \operatorname{sen} \beta t),
$$

onde $\alpha=\frac{-b}{2 a}$ e $\beta=\frac{\sqrt{4 a c-b^{2}}}{2 a}$, é uma solução complexa de (4.4), quando $b^{2}-4 a c<0$. Logo, pela Proposição 6, deduzimos que

$$
y_{1}(t)=e^{\alpha t} \cos \beta t \quad \text { e } \quad y_{2}(t)=e^{\alpha t} \operatorname{sen} \beta t
$$

são soluções reais de (4.4).

Vejamos agora que $y_{1}$ e $y_{2}$ são soluções linearmente independentes. Para isso, note que o Wronskiano de $y_{1}$ e $y_{2}$ é dado por

$$
\begin{aligned}
W\left[y_{1}, y_{2}\right](t) & =\left|\begin{array}{cc}
e^{\alpha t} \cos \beta t & e^{\alpha t} \operatorname{sen} \beta t \\
\alpha e^{\alpha t} \cos \beta t-\beta e^{\alpha t} \operatorname{sen} \beta t & \alpha e^{\alpha t} \operatorname{sen} \beta t+\beta e^{\alpha t} \cos \beta t
\end{array}\right| \\
& =\alpha e^{2 \alpha t} \cos \beta t \operatorname{sen} \beta t+\beta e^{2 \alpha t} \cos ^{2} \beta t-\alpha e^{2 \alpha t} \cos \beta t \operatorname{sen} \beta t+\beta e^{2 \alpha t} \operatorname{sen}^{2} \beta t \\
& =\beta e^{2 \alpha t} \neq 0,
\end{aligned}
$$

para todo $t$, pois $\beta \neq 0$. Logo, $y_{1}$ e $y_{2}$ são soluções linearmente independentes e a solução geral da EDO será dada por

$$
y(t)=e^{\alpha t}\left(c_{1} \cos \beta t+c_{2} \operatorname{sen} \beta t\right) .
$$

Observação 28. É natural questionar se $e^{\lambda_{2} t}$ originará outras duas soluções reais para (4.4), entretanto isso não ocorre, pois

$$
e^{\lambda_{2} t}=e^{(\alpha-i \beta) t}=e^{\alpha t}(\cos \beta t-i \operatorname{sen} \beta t)
$$


e, portanto, denotando por $\mathfrak{R}\left(e^{\lambda_{2} t}\right)$ e $\mathfrak{I}\left(e^{\lambda_{2} t}\right)$ a parte real e a parte imaginária de $e^{\lambda_{2} t}$, respectivamente, obtemos

$$
\begin{gathered}
\tilde{y}_{1}(t)=\mathfrak{R}\left(e^{\lambda_{2} t}\right)=e^{\alpha t} \cos \beta t=y_{1}(t), \\
\tilde{y}_{2}(t)=\mathfrak{I}\left(e^{\lambda_{2} t}\right)=-e^{\alpha t} \operatorname{sen} \beta t=-y_{2}(t) .
\end{gathered}
$$

Exemplo 41. Encontremos a solução real do P.V.I.

$$
\left\{\begin{array}{l}
y^{\prime \prime}-4 y^{\prime}+13 y=0 \\
y(0)=1 \\
y^{\prime}(0)=5
\end{array}\right.
$$

A equação característica dessa EDO é $\lambda^{2}-4 \lambda+13=0$, que tem raízes complexas

$$
\lambda_{1}=2+3 i \quad \text { e } \quad \lambda_{2}=2-3 i
$$

Assim,

$$
e^{\lambda_{1} t}=e^{(2+3 i) t}=e^{2 t}(\cos 3 t+i \operatorname{sen} 3 t)
$$

é uma solução complexa da EDO dada. Logo, pela Proposição 6, sabemos que

$$
y_{1}(t)=\mathfrak{R}\left(e^{\lambda_{1} t}\right)=e^{2 t} \cos 3 t \quad \text { e } \quad y_{2}(t)=\mathfrak{I}\left(e^{\lambda_{1} t}\right)=e^{2 t} \operatorname{sen} 3 t
$$

são soluções reais linearmente independentes da EDO e, assim, a solução geral é dada por

$$
y(t)=e^{2 t}\left(c_{1} \cos 3 t+c_{2} \operatorname{sen} 3 t\right)
$$

onde $c_{1}$ e $c_{2}$ são constantes reais. Aplicando a primeira condição inicial temos

$$
y(0)=e^{0}\left(c_{1} \cos 0+c_{2} \operatorname{sen} 0\right) \Rightarrow c_{1}=1
$$

e, portanto,

$$
y(t)=e^{2 t}\left(\cos 3 t+c_{2} \operatorname{sen} 3 t\right)
$$

o que implica $y^{\prime}(t)=2 e^{2 t}\left(\cos 3 t+c_{2} \operatorname{sen} 3 t\right)+e^{2 t}\left(-3 \operatorname{sen} 3 t+3 c_{2} \cos 3 t\right)$. Logo, aplicando a segunda condição inicial temos

$$
y^{\prime}(0)=2 e^{0}\left(\cos 0+c_{2} \operatorname{sen} 0\right)+e^{0}\left(-3 \operatorname{sen} 0+3 c_{2} \cos 0\right) \Rightarrow c_{2}=1 .
$$

Assim, a solução do P.V.I. é dada por

$$
y(t)=e^{2 t}(\cos 3 t+\operatorname{sen} 3 t) .
$$




\subsection{A Equação Não Homogênea}

Consideremos agora a equação linear não homogênea

$$
y^{\prime \prime}+a(t) y^{\prime}+b(t) y=g(t), \quad[\text { L.N.H. }]
$$

onde $a(t), b(t)$ e $g(t)$ são funções contínuas num intervalo $I$ e $g(t) \neq 0$. É interessante observar que nos fenômenos físicos descritos por uma equação dessa forma, o termo $g(t)$, geralmente, representa um "agente externo" atuando sobre o sistema.

Para a equação linear de primeira ordem não homogênea, $y^{\prime}+a(t) y=b(t)$, vimos que a solução geral é composta de duas partes:

I) a solução geral da equação homogênea associada $y^{\prime}+a(t) y=0$;

II) uma solução particular da equação não homogênea $y^{\prime}+a(t) y=b(t)$.

Veremos a seguir que este fato também é verdadeiro para as equações lineares de segunda ordem.

Teorema 8. Sejam $y_{1}(t)$ e $y_{2}(t)$ soluções linearmente independentes da equação linear homogênea $y^{\prime \prime}+a(t) y^{\prime}+b(t) y=0$ [L.H.] e seja $\varphi(t)$ uma solução particular da equação linear não homogênea $y^{\prime \prime}+a(t) y^{\prime}+b(t) y=g(t)$ [L.N.H.]. Então, toda solução de [L.N.H.] é da forma

$$
y(t)=c_{1} y_{1}(t)+c_{2} y_{2}(t)+\varphi(t)
$$

onde $c_{1}$ e $c_{2}$ são constantes reais.

Demonstração. Primeiramente, veja que, se $\varphi_{1}$ e $\varphi_{2}$ são soluções de [L.N.H.], então a função $\psi(t)=\varphi_{1}(t)-\varphi_{2}(t)$ é solução de [L.H.]. De fato, substituindo $\psi$ em [L.H.] temos que

$$
\begin{aligned}
\left(\varphi_{1}-\varphi_{2}\right)^{\prime \prime}+a(t)\left(\varphi_{1}-\varphi_{2}\right)^{\prime}+b(t)\left(\varphi_{1}-\varphi_{2}\right)= & \left(\varphi_{1}^{\prime \prime}+a(t) \varphi_{1}^{\prime}+b(t) \varphi_{1}\right) \\
& -\left(\varphi_{2}^{\prime \prime}+a(t) \varphi_{2}^{\prime}+b(t) \varphi_{2}\right) \\
= & g(t)-g(t)=0 .
\end{aligned}
$$

Logo, se $y(t)$ é uma solução de [L.N.H.], então $\psi(t)=y(t)-\varphi(t)$ é solução de [L.H.]. Por outro lado, como toda solução de [L.H.] é da forma $c_{1} y_{1}(t)+c_{2} y_{2}(t)$, concluímos que $\psi(t)=c_{1} y_{1}(t)+c_{2} y_{2}(t)$. Ou seja, $y(t)-\varphi(t)=c_{1} y_{1}(t)+c_{2} y_{2}(t)$, o que mostra que

$$
y(t)=c_{1} y_{1}(t)+c_{2} y_{2}(t)+\varphi(t) .
$$

O teorema acima reduz nosso problema de encontrar todas as soluções de [L.N.H.] ao problema de encontrar duas soluções linearmente independentes de [L.H.] e uma solução particular de [L.N.H.]. Ou seja, devemos encontrar a solução geral da equação [L.H.] associada e uma solução particular de [L.N.H.]. 
Definição 23. A expressão (4.6) é chamada solução geral de [L.N.H.].

Exemplo 42. Encontremos a solução geral de $y^{\prime \prime}-y=-t$.

Primeiramente, determinemos duas soluções linearmente independentes da equação homogênea associada $y^{\prime \prime}-y=0$, cuja equação característica é $\lambda^{2}-1=0$. Esta equação possui raízes $\lambda_{1}=1$ e $\lambda_{2}=-1$ e, assim, $y_{1}(t)=e^{t}$ e $y_{2}(t)=e^{-t}$ são duas soluções linearmente independentes de [L.H.]. Obviamente $\varphi(t)=t$ é uma solução particular de [L.N.H.]. Logo, pelo Teorema 8 , a solução geral da equação $y^{\prime \prime}-y=-t$ é

$$
y(t)=c_{1} e^{t}+c_{2} e^{-t}+t
$$

Exemplo 43. Sejam $\varphi_{1}(t)=t^{2}, \varphi_{2}(t)=t^{2}+e^{2 t}$ e $\varphi_{3}(t)=1+t^{2}+e^{2 t}$ três soluções de uma equação [L.N.H.] de segunda ordem. Encontremos a solução geral dessa equação.

Note que as funções $\varphi_{2}(t)-\varphi_{1}(t)=e^{2 t}$ e $\varphi_{3}(t)-\varphi_{2}(t)=1$ são duas soluções linearmente independentes da equação homogênea associada, pois $W(t)=\left|\begin{array}{cc}e^{2 t} & 1 \\ 2 e^{2 t} & 0\end{array}\right|=-2 e^{2 t} \neq 0, \forall t$. Logo, a solução geral de EDO é

$$
y(t)=c_{1}+c_{2} e^{2 t}+t^{2} .
$$

Observe que no Exemplo 43 podemos considerar qualquer uma das soluções particulares. Basta colocar os termos corretos em evidência.

Veremos a seguir dois métodos que permitem determinar uma solução particular de uma equação linear não homogênea.

\subsubsection{Método dos Coeficientes a Determinar}

Estudemos a equação

$$
a y^{\prime \prime}+b y^{\prime}+c y=g(t),
$$

onde $a, b$ e $c$ são constantes reais e $g(t)$ é uma função exponencial, ou um polinômio, ou uma das funções seno ou cosseno. Nesses casos veremos que não é difícil encontrar uma solução particular. O método também se aplica ao produto de tais funções, ou seja,

$$
g(t)=e^{\alpha t}\left(a_{0}+a_{1} t+\cdots+a_{n} t^{n}\right)\left(b_{1} \operatorname{sen} \beta t+b_{2} \cos \beta t\right) .
$$

Antes de estudarmos um procedimento geral vejamos os seguintes exemplos.

Exemplo 44. Encontremos uma solução particular da equação $y^{\prime \prime}-3 y^{\prime}-4 y=e^{5 t}$.

Tentaremos $y_{p}(t)=A e^{5 t}$, sendo assim $y_{p}^{\prime}(t)=5 A e^{5 t}$ e $y_{p}^{\prime \prime}(t)=25 A e^{5 t}$. Substituindo na equação temos $25 A e^{5 t}-15 A e^{5 t}-4 A e^{5 t}=6 A e^{5 t}=e^{5 t}$, portanto, $A=\frac{1}{6}$ e assim

$$
y_{p}(t)=\frac{1}{6} e^{5 t} .
$$


Exemplo 45. Encontremos uma solução particular da equação $y^{\prime \prime}+3 y^{\prime}+2 y=3 t$.

Queremos encontrar uma função $y_{p}(t)$ tal que a soma dos termos $y_{p}^{\prime \prime}, 3 y_{p}^{\prime}$ e $2 y_{p}$ seja igual a $3 t$. Uma opção seria procurarmos uma função da forma $y_{p}(t)=A t$. Mas ela não funciona, pois

$$
y_{p}(t)=A t \Rightarrow y_{p}^{\prime}(t)=A \Rightarrow y_{p}^{\prime \prime}(t)=0
$$

e, então,

$$
y_{p}^{\prime \prime}+3 y_{p}^{\prime}+2 y_{p}=0+3 A+2 A t .
$$

Logo, se $y_{p}^{\prime \prime}+3 y_{p}^{\prime}+2 y_{p}=3 A+2 A t=3 t$ devemos ter $A=0$ e $A=\frac{3}{2}$, o que é impossível. Por outro lado, se tentarmos $y_{p}(t)=A t+B$, temos que

$$
y_{p}(t)=A t+B \Rightarrow y_{p}^{\prime}(t)=A \Rightarrow y_{p}^{\prime \prime}(t)=0,
$$

e, portanto,

$$
y_{p}^{\prime \prime}+3 y_{p}^{\prime}+2 y_{p}=0+3 A+2(A t+B) .
$$

Assim,

$$
y_{p}^{\prime \prime}+3 y_{p}^{\prime}+2 y_{p}=(3 A+B)+2 A t=3 t \Rightarrow A=\frac{3}{2} \quad \text { e } \quad B=-\frac{9}{2} .
$$

Desse modo, uma solução particular da equação é

$$
y_{p}(t)=\frac{3 t}{2}-\frac{9}{2} .
$$

Exemplo 46. Encontremos uma solução particular da equação $y^{\prime \prime}-3 y^{\prime}+2 y=\operatorname{sen} t$.

Queremos encontrar uma função $y_{p}(t)$ tal que a soma dos termos $y_{p}^{\prime \prime},-3 y_{p}^{\prime}$ e $2 y_{p}$ seja igual a sent. É natural pensar na função $y_{p}(t)=A$ sent . Entretanto, em sua derivada surgirá $\cos t$ e não conseguiremos a igualdade com a função sent. Tentamos, então, $y_{p}(t)=A \operatorname{sen} t+B \cos t$. Assim,

$$
y_{p}^{\prime}(t)=A \cos t-B \operatorname{sen} t \quad \text { e } \quad y_{p}^{\prime \prime}(t)=-A \operatorname{sen} t-B \cos t
$$

Portanto,

$$
\begin{aligned}
y_{p}^{\prime \prime}-3 y_{p}^{\prime}+2 y_{p} & =-A \operatorname{sen} t-B \cos t-3(A \cos t-B \operatorname{sen} t)+2(A \operatorname{sen} t+B \cos t) \\
& =(A+3 B) \operatorname{sen} t+(-3 A+B) \cos t .
\end{aligned}
$$

Assim,

$$
y_{p}^{\prime \prime}-3 y_{p}^{\prime}+2 y_{p}=(A+3 B) \operatorname{sen} t+(-3 A+B) \cos t=\operatorname{sen} t,
$$

o que implica que

$$
\left\{\begin{array} { l } 
{ A + 3 B = 1 } \\
{ - 3 A + B = 0 }
\end{array} \Rightarrow \left\{\begin{array}{l}
A=\frac{1}{10} \\
B=\frac{3}{10}
\end{array} .\right.\right.
$$

Desse modo, uma solução particular da equação é

$$
y_{p}(t)=\frac{1}{10} \operatorname{sen} t+\frac{3}{10} \cos t
$$


Os exemplos acima motivam os procedimentos que serão apresentados a seguir para os casos em que $g(t)$ possui uma das formas:

I) $P_{n}(t)=a_{n} t^{n}+a_{n-1} t^{n-1}+\cdots+a_{1} t+a_{0}$,

II) $e^{\alpha t} P_{n}(t)$,

III) $e^{\alpha t} P_{n}(t) \operatorname{sen} \beta t$ ou $e^{\alpha t} P_{n}(t) \cos \beta t$,

IV) combinações lineares das anteriores.

$1^{0}$ Caso: $\operatorname{Se} g(t)=P_{n}(t), a_{n} \neq 0$, ficamos com a equação diferencial

$$
a y^{\prime \prime}+b y^{\prime}+c y=a_{n} t^{n}+a_{n-1} t^{n-1}+\cdots+a_{1} t+a_{0} .
$$

Assim, procuramos $y_{p}(t)$ de modo que a combinação $a y_{p}^{\prime \prime}+b y_{p}^{\prime}+c y_{p}$ seja um polinômio de grau $n$. Para isso, tomamos

$$
y_{p}(t)=A_{n} t^{n}+A_{n-1} t^{n-1}+\cdots+A_{1} t+A_{0}
$$

onde os coeficientes $A_{0}, A_{1}, \ldots, A_{n}$ devem ser determinados. Substituindo na equação (4.7) temos

$$
\begin{aligned}
& a\left[n(n-1) A_{n} t^{n-2}+(n-1)(n-2) A_{n-1} t^{n-3}+\cdots 6 A_{3} t+2 A_{2}\right] \\
+ & b\left[n A_{n} t^{n-1}+(n-1) A_{n-1} t^{n-2}+\cdots+2 A_{2} t+A_{1}\right] \\
+ & c\left[A_{n} t^{n}+A_{n-1} t^{n-1}+\cdots+A_{1} t+A_{0}\right] \\
= & a_{n} t^{n}+a_{n-1} t^{n-1}+\cdots+a_{1} t+a_{0} .
\end{aligned}
$$

Igualando os coeficientes obtemos

$$
\left\{\begin{array}{l}
c A_{n}=a_{n} \\
c A_{n-1}+n b A_{n}=a_{n-1} \\
c A_{n-2}+(n-1) b A_{n-1}+n(n-1) a A_{n}=a_{n-2} \\
\vdots \\
c A_{0}+b A_{1}+2 a A_{2}=a_{0} .
\end{array}\right.
$$

Se $c \neq 0$, pela primeira equação de (4.8) determinamos $A_{n}$. Logo, pela segunda equação determinamos $A_{n-1} \mathrm{e}$, assim, sucessivamente até determinarmos todos os $A_{i}, i=0,1, \ldots, n$.

Se $c=0$ e $b \neq 0$, então $a y_{p}^{\prime \prime}+b y_{p}^{\prime}$ é um polinômio de grau $n-1$, enquanto que $P_{n}(t)$ é um polinômio de grau $n$ e, assim, é impossível resolver (4.7). Logo, para garantir que $a y_{p}^{\prime \prime}+b y_{p}^{\prime}$ seja um polinômio de grau $n$, devemos escolher $y_{p}(t)$ como sendo um polinômio de grau $n+1$. Assim, tomamos

$$
y_{p}(t)=t\left(A_{n} t^{n}+A_{n-1} t^{n-1}+\cdots+A_{1} t+A_{0}\right)
$$


e procedemos como anteriormente. Observe que omitimos o termo constante, pois $y=$ constante é uma solução da equação homogênea associada, $a y^{\prime \prime}+b y^{\prime}=0$.

Analogamente, se $b=c=0$ tomamos

$$
y_{p}(t)=t^{2}\left(A_{n} t^{n}+A_{n-1} t^{n-1}+\cdots+A_{1} t+A_{0}\right)
$$

e procedemos como antes.

Exemplo 47. Encontremos uma solução particular da equação $y^{\prime \prime}+2 y^{\prime}=1+t^{2}$.

Note que, nesta $\mathrm{EDO}, c=0$ e $b \neq 0$, portanto tomamos

$$
y_{p}(t)=t\left(A t^{2}+B t+C\right) \text {. }
$$

Então, temos

$$
\begin{aligned}
& y_{p}^{\prime}(t)=3 A t^{2}+2 B t+C, \\
& y_{p}^{\prime \prime}(t)=6 A t+2 B .
\end{aligned}
$$

Substituindo na equação obtemos

$$
6 A t+2 B+2\left(3 A t^{2}+2 B t+C\right)=6 A t^{2}+(6 A+4 B) t+2(B+C)=1+t^{2},
$$

o que implica que

$$
\left\{\begin{array} { l } 
{ 6 A = 1 } \\
{ 6 A + 4 B = 0 } \\
{ 2 ( B + C ) = 1 }
\end{array} \Rightarrow \left\{\begin{array}{l}
A=\frac{1}{6} \\
B=-\frac{1}{4} \\
C=\frac{3}{4}
\end{array} .\right.\right.
$$

Logo, $y_{p}(t)=\frac{1}{6} t^{3}-\frac{1}{4} t^{2}+\frac{3}{4} t$ é uma solução particular da equação dada.

$2^{\circ}$ Caso: Se $g(t)=e^{\alpha t} P_{n}(t)$, ficamos com a equação diferencial

$$
a y^{\prime \prime}+b y^{\prime}+c y=e^{\alpha t} P_{n}(t) .
$$

Note que se removermos o termo $e^{\alpha t}$ do segundo membro de (4.9), esta equação se torna igual à equação (4.7). Podemos fazer isto tomando $y=e^{\alpha t} v$. Então, $y^{\prime}=e^{\alpha t}\left(v^{\prime}+\alpha v\right)$ e $y^{\prime \prime}=e^{\alpha t}\left(v^{\prime \prime}+2 \alpha v^{\prime}+\alpha^{2} v\right)$. Substituindo em (4.9) e cancelando o termo $e^{\alpha t}$ em ambos os membros obtemos

$$
a\left(v^{\prime \prime}+2 \alpha v^{\prime}+\alpha^{2} v\right)+b\left(v^{\prime}+\alpha v\right)+c v=P_{n}(t),
$$

ou seja,

$$
a v^{\prime \prime}+(2 a \alpha+b) v^{\prime}+\left(a \alpha^{2}+b \alpha+c\right) v=P_{n}(t) .
$$

Desse modo, $y(t)=e^{\alpha t} v(t)$ é solução de (4.9) se, e somente se, $v(t)$ é solução de (4.10), e este é um problema que já sabemos resolver.

Veja que para encontrar uma solução particular $v(t)$ de (4.10) devemos analisar os seguintes casos: 
(1) $a \alpha^{2}+b \alpha+c \neq 0$;

(2) $a \alpha^{2}+b \alpha+c=0$, mas $2 a \alpha+b \neq 0$;

(3) $a \alpha^{2}+b \alpha+c=2 a \alpha+b=0$.

A condição (1) diz que $\alpha$ não é raiz da equação característica

$$
a \lambda^{2}+b \lambda+c=0
$$

ou seja, $e^{\alpha t}$ não é solução da equação homogênea $a y^{\prime \prime}+b y^{\prime}+c y=0$. Por outro lado, neste caso, temos que $v(t)=Q_{n}(t)$, onde $Q_{n}(t)=A_{n} t^{n}+A_{n-1} t^{n-1}+\cdots+A_{1} t+A_{0}$, e, portanto,

$$
y_{p}(t)=Q_{n}(t) e^{\alpha t}
$$

Já a condição (2) significa $\alpha$ é raiz simples da equação característica (4.11), ou seja, $e^{\alpha t}$ é solução da equação homogênea $a y^{\prime \prime}+b y^{\prime}+c y=0$, mas $t e^{\alpha t}$ não é. Por outro lado, neste caso, temos que $v(t)=t Q_{n}(t)$ e, desse modo,

$$
y_{p}(t)=t Q_{n}(t) e^{\alpha t}
$$

Finalmente, a condição (3) significa que $\alpha$ é raiz dupla da equação característica (4.11), ou seja, tanto $e^{\alpha t}$ quanto $t e^{\alpha t}$ são soluções da equação homogênea $a y^{\prime \prime}+b y^{\prime}+c y=0$. Por outro lado, neste caso, temos que $v(t)=t^{2} Q_{n}(t)$ e, então,

$$
y_{p}(t)=t^{2} Q_{n}(t) e^{\alpha t}
$$

Da descrição acima observamos que, para encontrar uma solução particular $y_{p}(t)$ de (4.9), podemos estudar a equação homogênea associada $a y^{\prime \prime}+b y^{\prime}+c y=0$ da seguinte forma:

(i) $\operatorname{Se} e^{\alpha t}$ não é solução da equação homogênea, então

$$
y_{p}(t)=Q_{n}(t) e^{\alpha t}
$$

(ii) Se $e^{\alpha t}$ é solução da equação homogênea, mas $t e^{\alpha t}$ não é, então

$$
y_{p}(t)=t Q_{n}(t) e^{\alpha t}
$$

(iii) Se $e^{\alpha t}$ e $t e^{\alpha t}$ são soluções da equação homogênea, então

$$
y_{p}(t)=t^{2} Q_{n}(t) e^{\alpha t}
$$


Exemplo 48. Encontremos uma solução particular da equação $y^{\prime \prime}-3 y^{\prime}+2 y=(4-6 t) e^{-t}$.

A equação característica $\lambda^{2}-3 \lambda+2=0$ possui como raízes $\lambda_{1}=1$ e $\lambda_{2}=2$. Assim, $y_{1}(t)=e^{t}$ e $y_{2}(t)=e^{2 t}$ são as duas soluções linearmente independentes da equação homogênea associada. Portanto, $e^{-t}$ não é solução da equação homogênea e, desse modo, tomamos

$$
y_{p}(t)=(A+B t) e^{-t} \text {. }
$$

Então, teremos

$$
\begin{aligned}
& y_{p}^{\prime}(t)=B e^{-t}-(A+B t) e^{-t}=(-A+B-B t) e^{-t} \\
& y_{p}^{\prime \prime}(t)=-B e^{-t}-(-A+B-B t) e^{-t}=(A-2 B+B t) e^{-t} .
\end{aligned}
$$

Substituindo na equação e cancelando o fator comum $e^{-t}$ obtemos

$$
A-2 B+B t-3(-A+B-B t)+2(A+B t)=6 A-5 B+6 B t=4-6 t
$$

o que implica que

$$
\left\{\begin{array} { l } 
{ 6 A - 5 B = 4 } \\
{ 6 B = - 6 }
\end{array} \Rightarrow \left\{\begin{array}{l}
A=-\frac{1}{6} \\
B=-1
\end{array} .\right.\right.
$$

Logo, $y_{p}(t)=\left(-\frac{1}{6}-t\right) e^{-t}$ é uma solução particular da equação dada.

Exemplo 49. Encontremos uma solução particular da equação $y^{\prime \prime}-3 y^{\prime}+2 y=(4-6 t) e^{t}$.

Como vimos no Exemplo 48, $e^{t}$ é solução da equação homogênea associada, entretanto $t e^{t}$ não é solução. Logo, tomamos $y_{p}(t)=t(A+B t) e^{t}$. Substituindo $y_{p}(t)$ na equação e cancelando o fator comum $e^{t}$ obtemos

$$
-A+2 B-2 B t=4-6 t \Rightarrow\left\{\begin{array} { l } 
{ - A + 2 B = 4 } \\
{ - 2 B = - 6 }
\end{array} \Rightarrow \left\{\begin{array}{l}
A=2 \\
B=3
\end{array} .\right.\right.
$$

Logo, $y_{p}(t)=t(2+3 t) e^{t}$ é uma equação particular da equação dada.

Exemplo 50. Dada a equação $y^{\prime \prime}-6 y^{\prime}+9 y=\left(6+12 t+12 t^{2}+40 t^{3}+42 t^{5}\right) e^{3 t}$, encontremos uma solução particular para ela.

A equação característica $\lambda^{2}-6 \lambda+9=0$ possui raízes $\lambda_{1}=\lambda_{2}=3$. Assim, $y_{1}(t)=e^{3 t}$ e $y_{2}(t)=t e^{3 t}$ são duas soluções linearmente independentes da equação homogênea associada. Logo, tomamos $y_{p}(t)=t^{2}\left(A_{0}+A_{1} t+A_{2} t^{2}+A_{3} t^{3}+A_{4} t^{4}+A_{5} t^{5}\right) e^{3 t}$. Como podemos observar, substituir esta expressão na equação dada seria muito trabalhoso. Neste caso, é muito mais prático proceder da seguinte forma. Tome $y(t)=e^{3 t} v(t)$. Então, teremos

$$
y^{\prime}(t)=\left(v^{\prime}+3 v\right) e^{3 t} \quad \text { e } \quad y^{\prime \prime}(t)=\left(v^{\prime \prime}+6 v^{\prime}+9 v\right) e^{3 t} .
$$

Substituindo na expressão e cancelando o fator comum $e^{3 t}$, obtemos

$$
v^{\prime \prime}=6+12 t+12 t^{2}+40 t^{3}+42 t^{5} .
$$


Logo, integrando duas vezes temos

$$
v(t)=3 t^{2}+2 t^{3}+t^{4}+2 t^{5}+t^{7}
$$

Portanto, uma solução particular da equação dada é

$$
\begin{aligned}
y_{p}(t) & =\left(3 t^{2}+2 t^{3}+t^{4}+2 t^{5}+t^{7}\right) e^{3 t} \\
& =t^{2}\left(3+2 t+t^{2}+2 t^{3}+t^{5}\right) e^{3 t}
\end{aligned}
$$

$3^{0}$ caso: Se $g(t)=e^{\alpha t} P_{n}(t) \operatorname{sen} \beta t$ (ou $g(t)=e^{\alpha t} P_{n}(t) \cos \beta t$ ) ficamos com a equação diferencial

$$
a y^{\prime \prime}+b y^{\prime}+c y=e^{\alpha t} P_{n}(t) \operatorname{sen} \beta t \quad(\text { ou } \cos \beta t) .
$$

Este problema pode ser reduzido ao anterior se notarmos que

1) $e^{i \beta t}=\cos \beta t+i \operatorname{sen} \beta t$

2) se $y(t)=u(t)+i v(t)$ é uma solução a valores complexos da equação

$$
a y^{\prime \prime}+b y^{\prime}+c y=g_{1}(t)+i g_{2}(t)
$$

onde $a, b$ e $c$ são constantes reais, então

$$
\left\{\begin{array}{l}
a u^{\prime \prime}+b u^{\prime}+c u=g_{1}(t) \\
a v^{\prime \prime}+b v^{\prime}+c v=g_{2}(t) .
\end{array}\right.
$$

Provemos que a afirmação 2 é verdadeira. Note que

$$
y(t)=u(t)+i v(t) \Rightarrow y^{\prime}(t)=u^{\prime}(t)+i v^{\prime}(t) \Rightarrow y^{\prime \prime}(t)=u^{\prime \prime}(t)+i v^{\prime \prime}(t) .
$$

Substituindo na equação original obtemos

$$
a\left(u^{\prime \prime}+i v^{\prime \prime}\right)+b\left(u^{\prime}+i v^{\prime}\right)+c(u+i v)=g_{1}+i g_{2},
$$

que é equivalente a

$$
\left(a u^{\prime \prime}+b u^{\prime}+c u\right)+i\left(a v^{\prime \prime}+b v^{\prime}+c v\right)=g_{1}+i g_{2} .
$$

Agora, para que dois números complexos sejam iguais sabemos que suas partes real e imaginária devem ser iguais. Logo, concluímos que

$$
\left\{\begin{array}{l}
a u^{\prime \prime}+b u^{\prime}+c u=g_{1}(t) \\
a v^{\prime \prime}+b v^{\prime}+c v=g_{2}(t) .
\end{array}\right.
$$

Tendo isso provado, temos que se $\varphi(t)=u(t)+i v(t)$ é uma solução particular da equação

$$
a y^{\prime \prime}+b y^{\prime}+c y=\left(a_{0}+a_{1} t+\cdots+a_{n} t^{n}\right) e^{(\alpha+i \beta) t}
$$


ou, equivalentemente, fazendo $e^{i \beta t}=\cos \beta t+i \operatorname{sen} \beta t$,

$$
a y^{\prime \prime}+b y^{\prime}+c y=e^{\alpha t}\left(a_{0}+a_{1} t+\cdots+a_{n} t^{n}\right) \cos \beta t+i e^{\alpha t}\left(a_{0}+a_{1} t+\cdots+a_{n} t^{n}\right) \operatorname{sen} \beta t,
$$

pela afirmação 2 acima, concluímos que $u(t)=\mathfrak{R}[\varphi(t)]$ é uma solução de

$$
a y^{\prime \prime}+b y^{\prime}+c y=e^{\alpha t}\left(a_{0}+a_{1} t+\cdots+a_{n} t^{n}\right) \cos \beta t,
$$

e $v(t)=\mathfrak{I}[\varphi(t)]$ é uma solução de

$$
a y^{\prime \prime}+b y^{\prime}+c y=e^{\alpha t}\left(a_{0}+a_{1} t+\cdots+a_{n} t^{n}\right) \operatorname{sen} \beta t .
$$

Exemplo 51. Encontremos uma solução particular da equação $y^{\prime \prime}-3 y^{\prime}+2 y=20 \operatorname{sen} 2 t$.

Vamos determinar $y_{p}(t)$ como sendo a parte imaginária de uma solução a valores complexos $\varphi(t)$ da equação

$$
y^{\prime \prime}-3 y^{\prime}+2 y=20 e^{2 i t} .
$$

Como já vimos no Exemplo 48, $e^{2 i t}$ não é solução da equação homogênea associada. Logo, devemos tentar uma solução da forma $\varphi(t)=A e^{2 i t}$. Então, temos que

$$
\varphi^{\prime}(t)=2 i A e^{2 i t} \quad \text { e } \quad \varphi^{\prime \prime}(t)=-4 A e^{2 i t}
$$

Substituindo na equação ficamos com

$$
\left(-4 A e^{2 i t}\right)-3\left(2 i A e^{2 i t}\right)+2\left(A e^{2 i t}\right)=(-2-6 i) A e^{2 i t}=20 e^{2 i t},
$$

o que implica que

$$
A=\frac{20}{-2-6 i}=-1+3 i
$$

Logo,

$$
\varphi(t)=(-1+3 i) e^{2 i t}=(-1+3 i)(\cos 2 t+i \operatorname{sen} 2 t)=-\cos 2 t-3 \operatorname{sen} 2 t+i(3 \cos 2 t-\operatorname{sen} 2 t)
$$

e, portanto,

$$
y_{p}(t)=\mathfrak{I}[\varphi(t)]=3 \cos 2 t-\operatorname{sen} 2 t
$$

é uma solução particular da equação dada.

$4^{0}$ caso: Seja $g(t)$ uma combinação linear de funções dos tipos descritos nos casos anteriores. Podemos resolver este caso usando o Princípio da Superposição de Soluções que veremos a seguir.

Teorema 9. (Princípio da Superposição de Soluções) Suponha que $\varphi_{1}$ seja solução da equação $a y^{\prime \prime}+b y^{\prime}+c y=g_{1}(t)$ e que $\varphi_{2}$ seja solução da equação $a y^{\prime \prime}+b y^{\prime}+c y=g_{2}(t)$. Se $\alpha_{1}$ e $\alpha_{2}$ são constantes, então a função $\varphi(t)=\alpha_{1} \varphi_{1}(t)+\alpha_{2} \varphi_{2}(t)$ é solução da equação

$$
a y^{\prime \prime}+b y^{\prime}+c y=\alpha_{1} g_{1}(t)+\alpha_{2} g_{2}(t) .
$$


Demonstração. Substituindo $\varphi(t)$ na equação obtemos

$$
\begin{aligned}
a \varphi^{\prime \prime}+b \varphi^{\prime}+c \varphi & =a\left(\alpha_{1} \varphi_{1}+\alpha_{2} \varphi_{2}\right)^{\prime \prime}+b\left(\alpha_{1} \varphi_{1}+\alpha_{2} \varphi_{2}\right)^{\prime}+c\left(\alpha_{1} \varphi_{1}+\alpha_{2} \varphi_{2}\right) \\
& =a \alpha_{1} \varphi_{1}^{\prime \prime}+a \alpha_{2} \varphi_{2}^{\prime \prime}+b \alpha_{1} \varphi_{1}^{\prime}+b \alpha_{2} \varphi_{2}^{\prime}+c \alpha_{1} \varphi_{1}+c \alpha_{2} \varphi_{2} \\
& =\alpha_{1}\left(a \varphi_{1}^{\prime \prime}+b \varphi_{1}^{\prime}+c \varphi_{1}\right)+\alpha_{2}\left(a \varphi_{2}^{\prime \prime}+b \varphi_{2}^{\prime}+c \varphi_{2}\right) .
\end{aligned}
$$

Como, por hipótese, sabemos que $\varphi_{1}$ é solução de $a y^{\prime \prime}+b y^{\prime}+c y=g_{1}(t)$ e $\varphi_{2}$ é solução de $a y^{\prime \prime}+b y^{\prime}+c y=g_{2}(t)$, temos que

$$
\alpha_{1}\left(a \varphi_{1}^{\prime \prime}+b \varphi_{1}^{\prime}+c \varphi_{1}\right)+\alpha_{2}\left(a \varphi_{2}^{\prime \prime}+b \varphi_{2}^{\prime}+c \varphi_{2}\right)=\alpha_{1} g_{1}(t)+\alpha_{2} g_{2}(t)
$$

ou seja,

$$
a \varphi^{\prime \prime}(t)+b \varphi^{\prime}(t) c \varphi(t)=\alpha_{1} g_{1}(t)+\alpha_{2} g_{2}(t),
$$

o que prova o resultado.

Exemplo 52. Dada a equação $y^{\prime \prime}-3 y^{\prime}+2 y=(4-6 t) e^{t}+20 \operatorname{sen} 2 t$, encontremos uma solução particular para ela.

Para encontrar uma solução particular dessa equação devemos procurar uma solução particular $y_{p_{1}}(t)$ da equação

$$
y^{\prime \prime}-3 y^{\prime}+2 y=(4-6 t) e^{-t}
$$

e uma solução particular $y_{p_{2}}(t)$ da equação

$$
y^{\prime \prime}-3 y^{\prime}+2 y=20 \operatorname{sen} 2 t
$$

e, então, somar as duas soluções. Do Exemplo 49, sabemos que $y_{p_{1}}=t(2+3 t) e^{t}$ e do Exemplo 51 que $y_{p_{2}}=3 \cos 2 t-\operatorname{sen} 2 t$. Logo,

$$
y_{p}(t)=y_{p 1}+y_{p 2}=t(2+3 t) e^{t}+3 \cos 2 t-\operatorname{sen} 2 t .
$$

\subsubsection{Método da Variação de Parâmetros}

A principal vantagem deste método é o fato de ser um método mais geral para encontrar uma solução particular de uma EDO linear não homogênea de segunda ordem, pois se aplica também a equações com coeficientes variáveis. Entretanto, apresenta a desvantagem de, em geral, conduzir ao cálculo de integrais complicadas.

O método consiste em determinar uma solução particular da equação não homogênea

$$
y^{\prime \prime}+a(t) y^{\prime}+b(t) y=g(t), \quad[\text { L.N.H. }]
$$

a partir de duas soluções linearmente independentes da equação homogênea associada

$$
y^{\prime \prime}+a(t) y^{\prime}+b(t) y=0 . \quad[\text { L.H. }]
$$

Especificamente, dadas $y_{1}(t)$ e $y_{2}(t)$ duas soluções linearmente independentes da [L.H.], queremos encontrar uma solução particular $y_{p}(t)$ da [L.N.H.] da forma

$$
y_{p}(t)=u_{1}(t) y_{1}(t)+u_{2}(t) y_{2}(t) .
$$


Observação 29. Inicialmente, isso parece complicar nosso trabalho, pois estamos trocando o problema de encontrar uma função desconhecida $y_{p}(t)$ pelo problema de encontrar duas funções igualmente desconhecidas $u_{1}(t)$ e $u_{2}(t)$. Entretanto, trabalhando corretamente encontraremos $u_{1}(t)$ e $u_{2}(t)$ como as soluções de duas equações de primeira ordem muito simples.

Nosso objetivo, agora, é impor condições sobre $u_{1}(t)$ e $u_{2}(t)$ de modo que a expressão $y_{p}^{\prime \prime}+a(t) y_{p}^{\prime}+b(t) y_{p}$ se torne tão simples quanto possível. Derivando (4.12), obtemos

$$
y_{p}^{\prime}=u_{1} y_{1}^{\prime}+u_{2} y_{2}^{\prime}+u_{1}^{\prime} y_{1}+u_{2}^{\prime} y_{2}
$$

Para simplificar as expressões de $y_{p}^{\prime}$ e $y_{p}^{\prime \prime}$, vamos impor sobre $u_{1}(t)$ e $u_{2}(t)$ a condição

$$
u_{1}^{\prime} y_{1}+u_{2}^{\prime} y_{2}=0 \text {. }
$$

Assim, $y_{p}^{\prime}=u_{1} y_{1}^{\prime}+u_{2} y_{2}^{\prime}$ e, portanto,

$$
y_{p}^{\prime \prime}=u_{1}^{\prime} y_{1}^{\prime}+u_{1} y_{1}^{\prime \prime}+u_{2}^{\prime} y_{2}^{\prime}+u_{2} y_{2}^{\prime \prime}
$$

Substituindo $y_{p}, y_{p}^{\prime}$ e $y_{p}^{\prime \prime}$ na equação [L.N.H.] obtemos

$$
u_{1}^{\prime} y_{1}^{\prime}+u_{1} y_{1}^{\prime \prime}+u_{2}^{\prime} y_{2}^{\prime}+u_{2} y_{2}^{\prime \prime}+a\left(u_{1} y_{1}^{\prime}+u_{2} y_{2}^{\prime}\right)+b\left(u_{1} y_{1}+u_{2} y_{2}\right)=g
$$

e reagrupando convenientemente temos

$$
u_{1}^{\prime} y_{1}^{\prime}+u_{2}^{\prime} y_{2}^{\prime}+u_{1}\left(y_{1}^{\prime \prime}+a y_{1}^{\prime}+b y_{1}\right)+u_{2}\left(y_{2}^{\prime \prime}+a y_{2}^{\prime}+b y_{2}\right)=g
$$

Como $y_{1}$ e $y_{2}$ são soluções da equação homogênea, temos

$$
u_{1}^{\prime} y_{1}^{\prime}+u_{2}^{\prime} y_{2}^{\prime}=g
$$

Então, $y_{p}=u_{1} y_{1}+u_{2} y_{2}$ será solução da equação [L.N.H] se $u_{1}$ e $u_{2}$ satisfazem as condições

$$
\left\{\begin{array}{l}
y_{1} u_{1}^{\prime}+y_{2} u_{2}^{\prime}=0 \\
y_{1}^{\prime} u_{1}^{\prime}+y_{2}^{\prime} u_{2}^{\prime}=g,
\end{array}\right.
$$

que é um sistema linear em $u_{1}^{\prime}$ e $u_{2}^{\prime}$, cujo determinante da matriz dos coeficientes é $W\left[y_{1}, y_{2}\right](t)$, que é sempre diferente de zero, uma vez que $y_{1}$ e $y_{2}$ são soluções linearmente independentes da equação [L.H.]. Logo, a solução desse sistema é dada por

$$
u_{1}^{\prime}=-\frac{g y_{2}}{W} \quad \text { e } \quad u_{2}^{\prime}=\frac{g y_{1}}{W} .
$$

Finalmente, por integração, obtemos $u_{1}$ e $u_{2}$ e, consequentemente, $y_{p}$.

Exemplo 53. Encontremos uma solução particular da equação $y^{\prime \prime}-5 y^{\prime}+6 y=2 e^{t}$

Primeiramente, devemos encontrar duas soluções linearmente independentes da equação homogênea associada $y^{\prime \prime}-5 y^{\prime}+6 y=0$. A equação característica $\lambda^{2}-5 \lambda+6=0$ tem duas 
raízes reais distintas $\lambda_{1}=2$ e $\lambda_{2}=3$. Portanto, $y_{1}(t)=e^{2 t}$ e $y_{2}(t)=e^{3 t}$ são soluções linearmente independentes da homogênea associada, e ainda

$$
W\left[y_{1}, y_{2}\right](t)=\left|\begin{array}{cc}
e^{2 t} & e^{3 t} \\
2 e^{2 t} & 3 e^{3 t}
\end{array}\right|=e^{5 t} \neq 0, \quad \forall t \in \mathbb{R} .
$$

Logo, $y_{p}(t)=u_{1}(t) y_{1}(t)+u_{2}(t) y_{2}(t)$, onde

$$
\begin{aligned}
& u_{1}^{\prime}(t)=\frac{-g(t) y_{2}(t)}{W(t)}=\frac{-2 e^{t} e^{3 t}}{e^{5 t}}=\frac{-2}{e^{t}} \Rightarrow u_{1}(t)=\frac{2}{e^{t}}, \\
& u_{2}^{\prime}(t)=\frac{g(t) y_{1}(t)}{W(t)}=\frac{2 e^{t} e^{2 t}}{e^{5 t}}=\frac{2}{e^{2 t}} \Rightarrow u_{2}(t)=-\frac{1}{e^{2 t}} .
\end{aligned}
$$

Portanto, $u_{1}(t) y_{1}(t)=\frac{2}{e^{t}} e^{2 t}=2 e^{t}$ e $u_{2}(t) y_{2}(t)=-\frac{1}{e^{2 t}} e^{3 t}=-e^{t}$ e, então, uma solução particular da equação dada é

$$
y_{p}(t)=2 e^{t}-e^{t}=e^{t}
$$

\subsection{Equação de Cauchy-Euler}

Nesta seção, abordaremos, brevemente, a Equação de Cauchy-Euler, que pode ser considerada uma variação das equações diferenciais de $2^{\mathrm{a}}$ ordem com coeficientes constantes.

A Equação de Cauchy-Euler é a equação linear de segunda ordem da forma

$$
a t^{2} y^{\prime \prime}+b t y^{\prime}+c y=g(t)
$$

onde $a, b$ e $c$ são constantes, $a \neq 0$. Temos, de modo equivalente, a forma padrão

$$
y^{\prime \prime}+\frac{b}{a t} y^{\prime}+\frac{c}{a t^{2}} y=\frac{g(t)}{a t^{2}}, \quad t \neq 0 .
$$

Note que, na verdade, a Equação de Cauchy Euler apresenta uma forma mais geral do que as equações de $2^{\mathrm{a}}$ ordem com coeficientes constantes. No entanto, veremos a seguir que podemos tratá-las de maneira similar.

Especificamente, podemos procurar soluções da forma $y=t^{\lambda}$, pois os termos $t^{2} y^{\prime \prime}, t y^{\prime} \mathrm{e}$ $y$ são todos do mesmo tipo, ou seja,

$$
y=t^{\lambda}, \quad t y^{\prime}=\lambda t^{\lambda} \quad \text { e } \quad t^{2} y^{\prime \prime}=\lambda(\lambda-1) t^{\lambda} .
$$

Substituindo na equação homogênea associada a (4.13) obtemos

$$
a \lambda(\lambda-1) t^{\lambda}+b \lambda t^{\lambda}+c t^{\lambda}=\left[a \lambda^{2}+(b-a) \lambda+c\right] t^{\lambda}=0,
$$

o que implica que

$$
a \lambda^{2}+(b-a) \lambda+c=0,
$$


que também chamaremos de equação característica da equação homogênea associada a (4.13).

Assim como para equações homogêneas com coeficientes constantes, fazemos uma análise a respeito da quantidade de raízes da equação característica e da forma de duas soluções linearmente independentes da EDO.

É fácil verificar que, se a equação característica possui duas raízes reais distintas, então teremos duas soluções linearmente independentes dadas por $y_{1}(t)=t^{\lambda_{1}}$ e $y_{2}(t)=t^{\lambda_{2}}$. Se a equação característica possui duas raízes reais iguais, $\lambda=\lambda_{1}=\lambda_{2}$, então uma solução é dada por $y_{1}(t)=t^{\lambda}$ e usamos o método da redução de ordem para encontrar a segunda solução linearmente independente com $y_{1}(t)$, que será dada por $y_{2}(t)=t^{\lambda} \ln t$. Finalmente, se a equação característica possui duas raízes complexas, tomamos $\lambda_{1}=\alpha+i \beta$ e como

$$
t^{\alpha+i \beta}=t^{\alpha} t^{i \beta}=t^{\alpha} e^{i \beta \ln t}=t^{\alpha}[\cos (\beta \ln t)+i \operatorname{sen}(\beta \ln t)],
$$

teremos $y_{1}(t)=t^{\alpha} \cos (\beta \ln t)$ e $y_{2}(t)=t^{\alpha} \operatorname{sen}(\beta \ln t)$ duas soluções linearmente independentes da EDO.

Exemplo 54. Encontremos a solução geral da equação $t^{2} y^{\prime \prime}-2 t y^{\prime}+2 y=t^{3} e^{t}, t \neq 0$.

A equação característica da equação homogênea associada é dada por $\lambda^{2}-3 \lambda+2=0$ e possui duas raízes reais distintas $\lambda_{1}=1$ e $\lambda_{2}=2$. Portanto, $y_{1}(t)=t$ e $y_{2}(t)=t^{2}$ são duas soluções linearmente independentes da equação homogênea. Agora usaremos o método da variação dos parâmetros para achar uma solução particular. Para isso, devemos antes determinar o Wronskiano de $y_{1}$ e $y_{2}$,

$$
W(t)=\left|\begin{array}{cc}
t & t^{2} \\
1 & 2 t
\end{array}\right|=t^{2} \neq 0 .
$$

Então, usando a equação na forma padrão $y^{\prime \prime}-\frac{2}{t} y^{\prime}+\frac{2}{t^{2}} y=t e^{t}$, tomamos $u_{1}(t)$ e $u_{2}(t)$ tais que

$$
\begin{aligned}
& u_{1}^{\prime}(t)=\frac{-\left(t e^{t}\right)\left(t^{2}\right)}{t^{2}}=-t e^{t} \Rightarrow u_{1}(t)=-e^{t}(t-1), \\
& u_{2}^{\prime}(t)=\frac{\left(t e^{t}\right)(t)}{t^{2}}=e^{t} \Rightarrow u_{2}(t)=e^{t}
\end{aligned}
$$

Assim, uma solução particular da equação é dada por

$$
y_{p}(t)=-e^{t}(t-1) t+e^{t} t^{2}=t^{2} e^{t}-t^{2} e^{t}+t e^{t}=t e^{t} .
$$

Logo, a solução geral da equação dada é

$$
y(t)=c_{1} t+c_{2} t^{2}+t e^{t}
$$

Exemplo 55. Encontremos a solução geral da equação $t^{2} y^{\prime \prime}-2 t y^{\prime}+2 y=t^{-2}, t \neq 0$.

Do Exemplo 54, sabemos que $\lambda_{1}=1$ e $\lambda_{2}=2$, portanto, $y_{1}(t)=t$ e $y_{2}(t)=t^{2}$ são duas soluções linearmente independentes da equação homogênea associada. Agora, usaremos o 
método da variação dos parâmetros para achar uma solução particular. Para isso, devemos antes determinar o Wronskiano de $y_{1}$ e $y_{2}$, ou seja,

$$
W(t)=\left|\begin{array}{ll}
t & t^{2} \\
1 & 2 t
\end{array}\right|=t^{2} \neq 0 .
$$

Então, usando a equação na forma padrão $y^{\prime \prime}-\frac{2}{t} y^{\prime}+\frac{2}{t^{2}} y=t^{-4}$, tomamos $u_{1}(t)$ e $u_{2}(t)$ tais que

$$
\begin{aligned}
& u_{1}^{\prime}(t)=\frac{-\left(t^{-4}\right)\left(t^{2}\right)}{t^{2}}=-t^{-4} \Rightarrow u_{1}(t)=\frac{1}{3 t^{3}}, \\
& u_{2}^{\prime}(t)=\frac{\left(t^{-4}\right)(t)}{t^{2}}=t^{-5} \Rightarrow u_{2}(t)=-\frac{1}{4 t^{4}} .
\end{aligned}
$$

Assim, uma solução particular da equação é dada por

$$
y_{p}(t)=\frac{1}{3 t^{3}} t-\frac{1}{4 t^{4}} t^{2}=\frac{1}{3 t^{2}}-\frac{1}{4 t^{2}}=\frac{1}{12 t^{2}} .
$$

Logo, a solução geral da equação dada é

$$
y(t)=c_{1} t+c_{2} t^{2}+\frac{1}{12 t^{2}} .
$$

\subsection{Algumas Aplicações}

\subsubsection{Corpo em Queda Livre com Influência do Atrito}

Como já vimos, a variação da altura em que um corpo em queda livre se encontra do solo é dada pela equação

$$
m h^{\prime \prime}+k h^{\prime}=m g
$$

onde $m$ representa a massa do corpo, $g(>0)$ é a aceleração da gravidade e $k(>0)$ é a constante de resistência do ar.

Veja que a equação acima é uma equação linear de segunda ordem não homogênea com coeficientes constantes e, assim, sabemos encontrar sua solução, que será dada pela soma da solução geral da equação homogênea associada com uma solução particular da equação não homogênea.

Para encontrarmos a solução geral da equação homogênea associada, $m h^{\prime \prime}+k h^{\prime}=0$, consideremos sua equação característica

$$
m \lambda^{2}+k \lambda=0
$$

É fácil verificar que as raízes dessa equação são dadas por $\lambda_{1}=0$ e $\lambda_{2}=-\frac{k}{m}$. Assim,

$$
h_{1}(t)=e^{0 t}=1 \quad \text { e } \quad h_{2}(t)=e^{-\frac{k}{m} t}
$$


são duas soluções linearmente independentes da equação linear homogênea e sua solução geral é dada por

$$
c_{1} h_{1}(t)+c_{2} h_{2}(t)=c_{1}+c_{2} e^{-\frac{k}{m} t},
$$

onde $c_{1}$ e $c_{2}$ são constantes arbitrárias.

Por outro lado, podemos utilizar o Método dos Coeficientes a Determinar para encontrarmos uma solução particular para a equação não homogênea. Neste caso, devemos tomar $h_{p}(t)=t A$, o que implica que $h_{p}^{\prime}(t)=A$ e $h_{p}^{\prime \prime}(t)=0$. Logo, substituindo na equação temos que

$$
m \cdot 0+k A=m g \Rightarrow A=\frac{m g}{k}
$$

e, portanto, $h_{p}(t)=\frac{m g}{k} t$.

Assim a solução geral da equação é dada por

$$
h(t)=c_{1}+c_{2} e^{-\frac{k}{m} t}+\frac{m g}{k} t .
$$

Tomando a posição inicial do corpo, ou seja, a altura em que o corpo se encontra inicialmente como $h(0)=0$ e a velocidade inicial do corpo como $h^{\prime}(0)=v_{0}$, temos que

$$
h(0)=\frac{m g}{k} 0+c_{1}+c_{2} e^{\frac{-k 0}{m}}=0 \Rightarrow 0=c_{1}+c_{2} \Rightarrow c_{1}=-c_{2}
$$

$\mathrm{e}$

$$
h^{\prime}(0)=\frac{m g}{k}-\frac{k}{m} c_{2} e^{\frac{-k 0}{m}}=v_{0} \Rightarrow \frac{m}{k}\left(v_{0}-\frac{m g}{k}\right)=-c_{2} .
$$

Logo, a solução da EDO dada é

$$
h(t)=\frac{m g}{k} t+\frac{m}{k}\left(v_{0}-\frac{m g}{k}\right)\left(1-e^{\frac{-k t}{m}}\right) .
$$

Exemplo 56. Um objeto de $3 \mathrm{~kg}$ é solto do repouso de uma altura de $500 \mathrm{~m}$. Suponha que a força gravitacional seja constante, $\operatorname{com} g=9,81 \mathrm{~m} / \mathrm{s}^{2}$, e que a força, devido à resistência do ar, seja proporcional à velocidade do objeto, com constante de proporcionalidade $k=3 \mathrm{Ns} / \mathrm{m}$. Em que instante o objeto atingirá o solo?

Para calcular o instante $t$ em que o objeto atingirá o solo basta calcular em que instante ele terá percorrido $500 \mathrm{~m}$. Substituindo os dados do problema na equação (4.14) temos, num instante $t$ qualquer, que

$$
h(t)=\frac{(3)(9,81)}{3} t+\frac{3}{3}\left(0-\frac{(3)(9,81)}{3}\right)\left(1-e^{\frac{-3 t}{3}}\right)=9,81 t-9,81\left(1-e^{-t}\right) .
$$

Portanto, fazendo $h(t)=500$, obtemos

$$
500=9,81\left(t+e^{-t}\right)-9,81 \Rightarrow t+e^{-t} \cong 51,97 .
$$

Infelizmente, não podemos resolver de modo explícito esta equação, mas podemos fazer uma análise para encontrar uma aproximação. Como $t+e^{-t} \cong 51,97$, podemos supor que $51<t+e^{-t}<52$. Além disso, notando que $0<e^{-t}<1$ para todo $t>0$, podemos concluir que $50<t<52$. 
Observação 30. O modelo utilizado para um corpo em queda livre com influência do atrito pode ser adaptado para outros tipos de movimento em que haja influência de duas forças similares à gravidade e à resistência do ar. Vejamos um exemplo a seguir.

Exemplo 57. Um pequeno barco de $20 \mathrm{~kg}$ é rebocado a uma velocidade de $7 \mathrm{~m} / \mathrm{s}$. No instante em que o cabo do reboque é largado, um motor mantém o barco em movimento, exercendo uma força de 12 N. Sabendo que a resistência ao deslocamento, em N, é de 3 vezes a velocidade do barco em $\mathrm{m} / \mathrm{s}$, qual o descolamento do barco depois de 20 segundos?

A equação que modela o deslocamento do barco em função do tempo é dada por

$$
20 z^{\prime \prime}+3 z^{\prime}=12
$$

que corresponde a

$$
z^{\prime \prime}+0,15 z^{\prime}=0,6
$$

Primeiramente, devemos encontrar duas soluções linearmente independentes da equação homogênea associada $z^{\prime \prime}+0,15 z^{\prime}=0$, a equação característica dessa equação é $\lambda^{2}+0,15 \lambda=0$ e possui como raízes $\lambda_{1}=0$ e $\lambda_{2}=-0,15$. Então, as soluções linearmente independentes serão $z_{1}=1$ e $z_{2}=e^{-0,15 t}$. Agora, pelo método dos coeficientes a determinar é fácil verificar que $z_{p}(t)=4 t$ é uma solução particular da EDO. Assim, temos que a solução geral da EDO é

$$
z(t)=c_{1}+c_{2} e^{-0,15 t}+4 t
$$

e, aplicando as condições de que no início o barco estava a uma velocidade de $7 \mathrm{~m} / \mathrm{s}$ e a distância percorrida passou a ser contada do momento inicial, ou seja, $z(0)=0$ e $z^{\prime}(0)=7$, temos

$$
\begin{aligned}
z(0) & =c_{1}+c_{2}=0 \Rightarrow c_{1}=-c_{2}, \\
z^{\prime}(0) & =4-0,15 c_{2}=7 \Rightarrow c_{2}=-20 .
\end{aligned}
$$

Então, temos que a distância percorrida pelo barco em função do tempo é dada por

$$
z(t)=20-20 e^{-0,15 t}+4 t
$$

o que implica que $z(20)=99$. Ou seja, depois de 20 segundos o deslocamento do barco é de 99 metros.

\subsubsection{Aquecimento e Resfriamento de Prédios}

Considerando um prédio como sendo um único compartimento e $T(t)$ a temperatura no prédio no instante $t$, a taxa de variação da temperatura é determinada por todos os fatores que geram ou dissipam calor.

Consideremos os três fatores principais que afetam a temperatura dentro do prédio. $\mathrm{O}$ primeiro é o calor produzido por pessoas, lâmpadas e máquinas, que provoca um aumento na 
temperatura, indicado por $H(t)$, sempre não negativo. O segundo é o aquecimento ou resfriamento fornecido por aquecedor, ou ar-condicionado, e essa variação na temperatura será indicada por $U(t)$, que é positiva para aquecimento e negativa para resfriamento. O terceiro fator é o efeito da temperatura externa $M(t)$ sobre a interna $T(t)$ em que, pela lei de resfriamento de Newton, a taxa de variação de $T(t)$ é proporcional à diferença entre as temperaturas $M(t)$ e $T(t)$. Essa proporcionalidade é dada por uma constante positiva $K$, que depende das propriedades físicas do prédio, como número de portas e janelas, e o tipo de isolamento.

Resumindo a descrição acima, temos que

$$
\frac{d T}{d t}=K[M(t)-T(t)]+H(t)+U(t) .
$$

Entretanto, é mais comum encontrarmos situações em que o prédio seja dividido em mais do que um compartimento. Por exemplo, se considerarmos que existam dois compartimentos, digamos o ambiente $A$ e o ambiente $B$, podemos modelar a variação de temperatura nesses ambientes da seguinte forma

$$
\left\{\begin{array}{l}
\frac{d T_{A}}{d t}=K_{1}\left[M(t)-T_{A}(t)\right]+K_{2}\left[T_{B}(t)-T_{A}(t)\right]+H_{A}(t)+U_{A}(t) \\
\frac{d T_{B}}{d t}=K_{3}\left[M(t)-T_{B}(t)\right]+K_{2}\left[T_{A}(t)-T_{B}(t)\right]+H_{B}(t)+U_{B}(t)
\end{array},\right.
$$

em que $K_{1}$ é a constante de proporcionalidade de troca de calor entre o ambiente $A$ e o exterior do prédio, $K_{3}$ é a constante de proporcionalidade de troca de calor entre o ambiente $B$ e o exterior do prédio e $K_{2}$ é constante de proporcionalidade de troca de calor entre os ambientes $A$ e $B$.

Exemplo 58. Uma casa consiste em dois ambientes, a área do sotão $A$ e a área de estar $B$ que é resfriada por um ar-condicionado que remove $24000 \mathrm{Btu} / \mathrm{h}$. Suponha que a capacidade de calor da área $B$ é de $\frac{1}{2}^{\circ} \mathrm{F}$ por mil Btu, a constante de proporcionalidade entre a área externa e o ambiente $A$ é $\frac{1}{2}$, entre a área externa e o ambiente $B$ é $\frac{1}{4}$ e entre os ambientes $A$ e $B$ é $\frac{1}{4}$. Se a temperatura exterior permanece em $100^{\circ} \mathrm{F}$, a que temperatura chega a área $A$ ? $\mathrm{E}$ a área $B$ ?

Dos dados do problema temos que $K_{1}=\frac{1}{2}, K_{2}=K_{3}=\frac{1}{4}$.

Agora assumimos que $H_{A}(t)=H_{B}(t)=0$, e $U_{A}=0$, pois a área $A$ não é aquecida ou resfriada. Da física, sabemos que como a capacidade de calor da área $B$ é de $\frac{1}{2}^{\circ} \mathrm{F}$ por mil Btu e que essa área tem uma remoção de $24000 \mathrm{Btu} / \mathrm{h}$, então, $U_{B}(t)=-\left(\frac{1}{2}\right)\left(\frac{24000}{1000}\right)=-12$. Logo,

$$
\begin{aligned}
\frac{d T_{A}}{d t} & =0,5\left[100-T_{A}(t)\right]+0,25\left[T_{B}(t)-T_{A}(t)\right], \\
\frac{d T_{B}}{d t} & =0,25\left[100-T_{B}(t)\right]+0,25\left[T_{A}(t)-T_{B}(t)\right]-12,
\end{aligned}
$$

que podem ser reescritas como

$$
\begin{aligned}
\frac{d T_{A}}{d t} & =-0,75 T_{A}+0,25 T_{B}+50 \\
\frac{d T_{B}}{d t} & =-0,5 T_{B}+0,25 T_{A}+13
\end{aligned}
$$


Isolando $T_{B}$ em (4.15) temos

$$
T_{B}=\frac{T_{A}^{\prime}+0,75 T_{A}-50}{0,25} \Rightarrow T_{B}^{\prime}=\frac{T_{A}^{\prime \prime}+0,75 T_{A}^{\prime}}{0,25},
$$

e substituindo em (4.16) obtemos

$$
\frac{T_{A}^{\prime \prime}+0,75 T_{A}^{\prime}}{0,25}=-0,5\left(\frac{T_{A}^{\prime}+0,75 T_{A}-50}{0,25}\right)+0,25 T_{A}+13,
$$

o que implica que

$$
T_{A}^{\prime \prime}+1,25 T_{A}^{\prime}+0,3125 T_{A}=28,25,
$$

que é uma EDO linear de segunda ordem não homogenêa com coeficientes constantes e é equivalente a

$$
16 T_{A}^{\prime \prime}+20 T_{A}^{\prime}+5 T_{A}=452 .
$$

Então, tomando a equação característica da equação homogênea associada, temos

$$
16 \lambda^{2}+20 \lambda+5=0
$$

e, assim, $\lambda_{1} \cong-0,904$ e $\lambda_{2} \cong-0,345$. Portanto, $y_{1}(t)=e^{-0,904 t}$ e $y_{2}(t)=e^{-0,345 t}$ são duas soluções linearmente independentes da equação homogênea associada.

Agora, usando o método dos coeficientes a determinar podemos encontrar uma solução particular da equação dada. Devemos procurar $y_{p}=A$ e assim $y_{p}^{\prime}=y_{p}^{\prime \prime}=0$. Consequentemente, $A=\frac{452}{5}=90,4$ e $y_{p}=90,4$. Logo, a solução geral da equação (4.17) é dada por

$$
T_{A}(t)=90,4+c_{1} e^{-0,904 t}+c_{2} e^{-0,345 t},
$$

e daqui podemos notar que a temperatura da área $A$ tende a $90,4^{\circ} \mathrm{F}$.

Além disso, temos que

$$
T_{B}(t)=\frac{T_{A}^{\prime}-0,75 T_{A}-50}{0,25}=71,2-0,616 c_{1} e^{-0,904 t}+1,62 c_{2} e^{-0,342 t},
$$

o que implica que a temperatura da área $B$ tende a $71,2^{\circ} \mathrm{F}$.

Nesse exemplo apareceram as temperaturas $100^{\circ} \mathrm{F}, 90,4^{\circ} \mathrm{F}$ e $71,2^{\circ} \mathrm{F}$ que correspondem, respectivamente, a aproximadamente $37,8^{\circ} \mathrm{C}, 32,4^{\circ} \mathrm{C}$ e $21,8^{\circ} \mathrm{C}$.

\subsubsection{Um Modelo para Detecção de Diabetes}

A glicose é um dos mais importantes carboidratos, por ser usada como fonte de energia pela maior parte dos organismos. Para cada indivíduo existe uma concentração ideal de glicose no sangue e qualquer desvio excessivo acarreta certas condições patológicas, inclusive a morte.

Os níveis de glicose no sangue tendem a ser auto regulatórios, mas eles também são influenciados e controlados por uma ampla variedade de hormônios e outros metabólitos, sendo a insulina o principal deles. 
O modelo básico da concentração de glicose e de hormônios no sangue é descrito pelo seguinte sistema de EDOs

$$
\left\{\begin{array}{l}
\frac{d G}{d t}=F_{1}(G, H)+J(t), \\
\frac{d H}{d t}=F_{2}(G, H),
\end{array}\right.
$$

onde $G$ é a concentração de glicose no sangue, $H$ é a concentração hormonal líquida no sangue e $J$ é a taxa externa em que a concentração de glicose no sangue é aumentada. Muitas vezes temos interesse em descobrir os desvios de $G$ e $H$ de seus valores ideais.

Suponhamos que $G$ e $H$ assumam os valores ideais, $G_{0}$ e $H_{0}$, no tempo $t=t_{0}$, quando a pessoa está em jejum, isto é, $G\left(t_{0}\right)=G_{0}, H\left(t_{0}\right)=H_{0}, J\left(t_{0}\right)=0, F_{1}\left(G_{0}, H_{0}\right)=0$ e $F_{2}\left(G_{0}, H_{0}\right)=0$. Como estamos interessados em descobrir os desvios de $G$ e $H$ de seus valores ideais, consideramos as variáveis $g$ e $h$ que correspondem a esses desvios, ou seja, $g=G-G_{0}$ e $h=H-H_{0}$. Assim, o sistema (4.18) é dado, nas novas variáveis, por

$$
\left\{\begin{array}{l}
\frac{d g}{d t}=F_{1}\left(G_{0}+g, H_{0}+h\right)+J(t), \\
\frac{d h}{d t}=F_{2}\left(G_{0}+g, H_{0}+h\right) .
\end{array}\right.
$$

Para facilitar o estudo do sistema (4.19) utilizaremos uma aproximação das funções $F_{1}$ e $F_{2}$ em torno do ponto $\left(G_{0}, H_{0}\right)$. Esta aproximação é chamada de fórmula de Taylor de $2^{\mathrm{a}}$ ordem para funções de duas variáveis e é dada por

$$
F_{1}\left(G_{0}+g, H_{0}+h\right)=F_{1}\left(G_{0}, H_{0}\right)+\frac{\partial F_{1}\left(G_{0}, H_{0}\right)}{\partial G} g+\frac{\partial F_{1}\left(G_{0}, H_{0}\right)}{\partial H} h+e_{1}(g, h)
$$

$\mathrm{e}$

$$
F_{2}\left(G_{0}+g, H_{0}+h\right)=F_{2}\left(G_{0}, H_{0}\right)+\frac{\partial F_{2}\left(G_{0}, H_{0}\right)}{\partial G} g+\frac{\partial F_{2}\left(G_{0}, H_{0}\right)}{\partial H} h+e_{2}(g, h),
$$

onde $e_{1}(g, h)$ e $e_{2}(g, h)$ são os erros cometidos nas aproximações e envolvem derivadas parciais de $2^{\mathrm{a}}$ ordem de $F_{1}$ e $F_{2}$, respectivamente, no ponto $\left(G_{0}, H_{0}\right)$. Notando que estes erros são muito pequenos se estivermos muito próximos do ponto $\left(G_{0}, H_{0}\right)$, admitindo que $G$ e $H$ desviam-se muito pouco de $G_{0}$ e $H_{0}$, podemos desprezar os termos $e_{1}(g, h)$ e $e_{2}(g, h)$, e teremos uma boa aproximação do modelo original

$$
\begin{aligned}
\frac{d g}{d t} & =\frac{\partial F_{1}\left(G_{0}, H_{0}\right)}{\partial G} g+\frac{\partial F_{1}\left(G_{0}, H_{0}\right)}{\partial H} h+J(t) \\
\frac{d h}{d t} & =\frac{\partial F_{2}\left(G_{0}, H_{0}\right)}{\partial G} g+\frac{\partial F_{2}\left(G_{0}, H_{0}\right)}{\partial H} h .
\end{aligned}
$$

Agora, a fim de resolvermos o sistema (4.20) - (4.21) determinaremos os sinais de $\frac{\partial F_{1}\left(G_{0}, H_{0}\right)}{\partial G}, \frac{\partial F_{1}\left(G_{0}, H_{0}\right)}{\partial H}, \frac{\partial F_{2}\left(G_{0}, H_{0}\right)}{\partial G}$ e $\frac{\partial F_{2}\left(G_{0}, H_{0}\right)}{\partial H}$.

No que segue supomos que não há influência externa na concentração de glicose, isto é, $J(t)=0$.

Para $g>0$ (ou seja, a quantidade de glicose no sangue é maior que o ideal) e $h=0$ (ou seja, não há desvio na concentração de hormônios no sangue), a concentração de glicose no 
sangue diminuirá por meio da absorção pelos tecidos e do depósito do excesso de glicose no fígado. Então, temos $G^{\prime}<0$, o que implica $g^{\prime}<0$, e da equação (4.20) concluímos que $\frac{\partial F_{1}\left(G_{0}, H_{0}\right)}{\partial G}$ é negativo.

A mesma situação acima, $g>0$ e $h=0$, implica uma liberação de hormônios fazendo aumentar a concentração dos mesmos no sangue, então, $H^{\prime}>0$, o que implica $h^{\prime}>0$. Logo, da equação (4.21) concluímos que $\frac{\partial F_{2}\left(G_{0}, H_{0}\right)}{\partial G}$ é positivo.

Para $g=0$ (ou seja, não há desvio na concentração de glicose no sangue) e $h>0$ (ou seja, a quantidade de hormônios no sangue é maior que o ideal), a concentração de glicose no sangue diminuirá, pois o aumento da concentração de hormônios propicia a absorção de glicose pelos tecidos e do depósito do excesso de glicose no fígado. Então, temos $G^{\prime}<0$, o que implica $g^{\prime}<0$, e pela equação (4.20) concluímos que $\frac{\partial F_{1}\left(G_{0}, H_{0}\right)}{\partial H}$ é negativo.

A mesma situação acima, $g=0$ e $h>0$, implica uma diminuição na concentração de hormônios no sangue, pois o corpo tende a metabolizá-los. Então, $H^{\prime}<0$, o que implica $h^{\prime}<0$, e pela equação (4.21) concluímos que $\frac{\partial F_{2}\left(G_{0}, H_{0}\right)}{\partial H}$ é negativo.

Portanto, é possível escrever as equações (4.20) e (4.21) na forma

$$
\begin{aligned}
& \frac{d g}{d t}=-m_{1} g-m_{2} h+J(t), \\
& \frac{d h}{d t}=m_{4} g-m_{3} h,
\end{aligned}
$$

onde $m_{1}, m_{2}, m_{3}$ e $m_{4}$ são constantes positivas. Como podemos medir apenas a concentração de glicose no sangue, é interessante isolarmos $h$ na equação (4.22), ou seja,

$$
h=\frac{g^{\prime}+m_{1} g-J}{-m_{2}} \Rightarrow h^{\prime}=\frac{g^{\prime \prime}+m_{1} g^{\prime}-J^{\prime}}{-m_{2}}
$$

e substituindo na equação (4.23)

$$
\frac{g^{\prime \prime}+m_{1} g^{\prime}-J^{\prime}}{-m_{2}}=-m_{3} \frac{g^{\prime}+m_{1} g-J}{-m_{2}}+m_{4} g,
$$

ou seja,

$$
g^{\prime \prime}+\left(m_{1}+m_{3}\right) g^{\prime}+\left(m_{3} m_{1}+m_{2} m_{4}\right) g=m_{3} J+J^{\prime} .
$$

Para facilitar nosso estudo, podemos reescrever a equação acima na forma

$$
\frac{d^{2} g}{d t^{2}}+2 \alpha \frac{d g}{d t}+\omega^{2} g=S(t)
$$

onde $\alpha=\frac{m_{1}+m_{3}}{2}, \omega^{2}=m_{3} m_{1}+m_{2} m_{4}$ e $S(t)=m_{3} J+J^{\prime}$. Veja que o segundo membro da equação (4.24) é identicamente nulo exceto no intervalo de tempo muito pequeno em que a dose de glicose está sendo ingerida. Seja $t=0$ o instante no qual a dose de glicose foi completamente ingerida, então, para $t \geqslant 0, g(t)$ satisfaz a equação linear homogênea de segunda ordem

$$
\frac{d^{2} g}{d t^{2}}+2 \alpha \frac{d g}{d t}+\omega^{2} g=0
$$


cuja equação característica é dada por $\lambda^{2}+2 \alpha \lambda+\omega^{2}=0$. Daí segue que $\lambda=-\alpha \pm \sqrt{\alpha^{2}-\omega^{2}}$. Tomando $\beta=\sqrt{\alpha^{2}-\omega^{2}}$, estudemos os três possíveis casos para $\alpha^{2}-\omega^{2}$.

$1^{0}$ caso: $\left(\alpha^{2}-\omega^{2}>0\right)$

Neste caso, temos que a equação possui duas raízes reais distintas. Além disso, as duas raízes são negativas, pois $\alpha=\sqrt{\alpha^{2}}>\sqrt{\alpha^{2}-\omega^{2}}=\beta$, e a solução geral de (4.25) é dada por

$$
g(t)=c_{1} e^{\lambda_{1} t}+c_{2} e^{\lambda_{2} t},
$$

onde $\lambda_{1}=-\alpha+\beta, \lambda_{2}=-\alpha-\beta$, e $c_{1}, c_{2} \in \mathbb{R}$.

$$
\mathbf{2}^{\circ} \text { caso: }\left(\alpha^{2}-\omega^{2}=0\right)
$$

Neste caso, temos que a equação possui uma raiz real dupla, $\lambda=-\alpha$. Assim, temos que as duas soluções linearmente independentes são $g_{1}(t)=e^{-\alpha t}$ e $g_{2}(t)=t e^{-\alpha t}$. Desse modo, a solução geral de (4.25) é dada por

$$
g(t)=\left(c_{1}+c_{2} t\right) e^{-\alpha t},
$$

onde $c_{1}, c_{2} \in \mathbb{R}$.

$$
3^{0} \text { caso: }\left(\alpha^{2}-\omega^{2}<0\right)
$$

Aqui temos $\lambda_{1}=-\alpha+\beta i$ e $\lambda_{2}=-\alpha-\beta i$. Logo, como já foi visto, a solução geral de (4.25) é dada por

$$
g(t)=e^{-\alpha t}\left(c_{1} \cos (\beta t)+c_{2} \operatorname{sen}(\beta t)\right) .
$$

Veja que a equação (4.26) pode ser escrita de duas formas diferentes. Tomemos primeiramente $c_{1}=A \cos (\delta)$ e $c_{2}=A \operatorname{sen}(\boldsymbol{\delta})$. Substituindo em (4.26) ficamos com

$$
g(t)=A e^{-\alpha t} \cos (\beta t-\delta)
$$

Agora, tomando $c_{1}=A \operatorname{sen}(\gamma)$ e $c_{2}=A \cos (\gamma)$ e substituindo em (4.26) temos

$$
g(t)=A e^{-\alpha t} \operatorname{sen}(\beta t+\gamma)
$$

Logo, é fácil verificar que a solução (4.26) oscila entre $-A e^{-\alpha t}$ e $A e^{-\alpha t}$. Note ainda que nas duas situações acima $A=\sqrt{c_{1}^{2}+c_{2}^{2}}$. Além disso, na primeira situação temos $\delta=\arctan \left(\frac{c_{2}}{c_{1}}\right) \mathrm{e}$ na segunda $\gamma=\arctan \left(\frac{c_{1}}{c_{2}}\right)=\arctan \left(\frac{c_{2}}{c_{1}}\right)^{-1}$.

É importante notar também que em qualquer caso, a solução de (4.25) tende a zero quando $t$ tende ao infinito, o que está de acordo com o modelo proposto, pois se espera que a quantidade de glicemia no sangue volte ao valor ótimo e, assim, não haja desvio desse valor, ou seja, $g=0$, após aplicada uma dose de glicose.

Um problema deste modelo é que ele não considera variáveis como a adrenalina, que aumenta os níveis de glicose no sangue. Uma forma de diagnosticar o diabetes Mellitus é através 
do teste de tolerância à glicose (TTG), que é feito da seguinte maneira, o paciente, em jejum, recebe uma solução com 75 gramas de glicose e são realizadas coletas de sangue de acordo com o solicitado pelo médico, geralmente, uma após 2 horas. O fato é que há evidência indicando um aumento considerável nos níveis de adrenalina durante a recuperação do TTG, (antes das 2 horas), pois em alguns pacientes ocorre uma variação muito grande dos níveis de glicose no sangue, ficando abaixo do nível medido em jejum, que é considerado o valor ótimo. Logo, o corpo interpreta isso como uma emergência e secreta uma grande quantidade de adrenalina e, portanto, de glicose no sangue. A seguir apresentamos um exemplo de um modelo que envolve o TTG e a concentração de glicose no sangue de um determinado paciente.

Exemplo 59. A concentração de glicose em $100 \mathrm{ml}$ de sangue de um paciente satisfaz o P.V.I.

$$
\left\{\begin{array}{l}
G^{\prime \prime}+\frac{1}{20} G^{\prime}+\frac{1}{2500} G=\frac{75}{2500}, \\
G(0)=150, \\
G^{\prime}(0)=-3,75,
\end{array}\right.
$$

após absorver a quantidade de glicose proposta no TTG. Sabendo que a concentração de glicose ideal no sangue desse paciente é de $75 \mathrm{mg}$ de glicose / $100 \mathrm{ml}$ de sangue e que se após as 2 horas a concentração é inferior a $140 \mathrm{mg}$ de glicose / $100 \mathrm{ml}$ de sangue ele não é diabético, verifique se este paciente é diabético ou não. Após quanto tempo a concentração de glicose volta ao ideal?

Primeiramente, devemos encontrar a solução do P.V.I.. Para isso, precisamos encontrar as duas soluções linearmente independentes da equação homogênea

$$
G^{\prime \prime}+\frac{1}{20} G^{\prime}+\frac{1}{2500} G=0,
$$

cuja equação característica é $\lambda^{2}+\frac{1}{20} \lambda+\frac{1}{2500}=0$, com soluções $\lambda_{1}=-0,01$ e $\lambda_{2}=-0,04$. Portanto, as duas soluções linearmente independentes são $G_{1}(t)=c_{1} e^{-0,01 t}$ e $G_{2}(t)=c_{2} e^{-0,04 t}$. Além disso, obviamente temos que $G_{p}(t)=75$ é uma solução particular da equação não homogênea e, assim, a solução geral é dada por

$$
G(t)=75+c_{1} e^{-0,01 t}+c_{2} e^{-0,04 t} .
$$

Agora, aplicando as condições iniciais temos

$$
\begin{aligned}
G(0) & =75+c_{1}+c_{2} \Rightarrow c_{1}+c_{2}=75 \\
G^{\prime}(0) & =-0,01 c_{1}-0,04 c_{2} \Rightarrow-0,01 c_{1}-0,04 c_{2}=-3,75
\end{aligned}
$$

o que implica que $c_{1}=-25$ e $c_{2}=100$. Logo, a solução do P.V.I. é dada por

$$
G(t)=75+100 e^{-0,04 t}-25 e^{-0,01 t} .
$$

Portanto, calculando $G(120)$, obtemos

$$
G(120)=75+100 e^{-4,8}-25 e^{-1,2} \cong 75+0,823-7,53=68,293 .
$$


Assim, temos que esse paciente não é diabético, pois este valor é inferior a 140.

Além disso, temos que se $G(t)=75$, então

$$
75=75+100 e^{-0,04 t}-25 e^{-0,01 t} \Rightarrow \frac{e^{-0,01 t}}{e^{-0,04 t}}=4 \Rightarrow t \cong 46,21 .
$$

Assim, a concentração de glicose volta ao ideal após aproximadamente 46 minutos e 12 segundos. 
CAPÍTULO

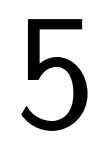

\section{O MÉTODO DE APROXIMAÇÕES DE EULER E UMA APLICAÇÃO NA EDUCAÇÃO BÁSICA}

Neste capítulo, apresentamos um método numérico para aproximar soluções de uma equação diferencial ordinária de $1^{\mathrm{a}}$ ordem. Especificamente, apresentamos o chamado método de Euler bem como o método de Euler aperfeiçoado. Em seguida, descreveremos como foi feita uma aplicação desses dois métodos com alguns alunos do ensino médio.

\subsection{O método de Euler}

Considerando o P.V.I. de primeira ordem

$$
\left\{\begin{array}{l}
\frac{d y}{d t}=f(t, y), \\
y\left(t_{0}\right)=y_{0}
\end{array}\right.
$$

lembramos de dois fatos importantes. Se $f$ e $\frac{\partial f}{\partial y}$ são contínuas, então o P.V.I. tem uma única solução $y=\phi(t)$ em algum intervalo contendo o ponto inicial $t=t_{0}$. Além disso, nem sempre é possível encontrar a solução $\phi$ manipulando diretamente a equação diferencial. Isto ocorre na maioria dos casos, ou seja, as soluções da maioria dos P.V.I.s de primeira ordem não podem ser encontradas por métodos analíticos, como os considerados no Capítulo 3 para alguns casos especiais de equações de primeira ordem (lineares, separáveis e exatas). Assim, é interessante se pudermos abordar o problema de outras maneiras. Veremos, neste capítulo, um método simples que gera aproximações numéricas de soluções para o P.V.I. (5.1). Este método foi desenvolvido por Euler por volta de 1768 e é conhecido como método da reta tangente ou método de Euler.

Para entender como o método de Euler funciona notamos, inicialmente, que o gráfico da solução do P.V.I. (5.1) contém o ponto $\left(t_{0}, y_{0}\right)$ e que a inclinação da reta tangente ao gráfico neste ponto é $f\left(t_{0}, y_{0}\right)$, pois $y^{\prime}\left(t_{0}\right)=f\left(t_{0}, y_{0}\right)$. Assim, a equação da reta tangente ao gráfico da 
solução em $\left(t_{0}, y_{0}\right)$ pode ser escrita como

$$
y=y_{0}+f\left(t_{0}, y_{0}\right)\left(t-t_{0}\right) .
$$

Considerando um intervalo suficientemente pequeno, sabe-se que a reta tangente é uma boa aproximação para a curva solução de (5.1). Então, para $t_{1}$ suficientemente próximo de $t_{0}$ podemos aproximar $\phi\left(t_{1}\right)$ pelo valor $y_{1}$, que obtemos substituindo $t=t_{1}$ na equação da reta tangente à curva solução em $\left(t_{0}, y_{0}\right)$, ou seja,

$$
y_{1}=y_{0}+f\left(t_{0}, y_{0}\right)\left(t_{1}-t_{0}\right) \text {. }
$$

Veja na figura a seguir a representação gráfica desta situação.

Figura 6 - Uma aproximação pela reta tangente

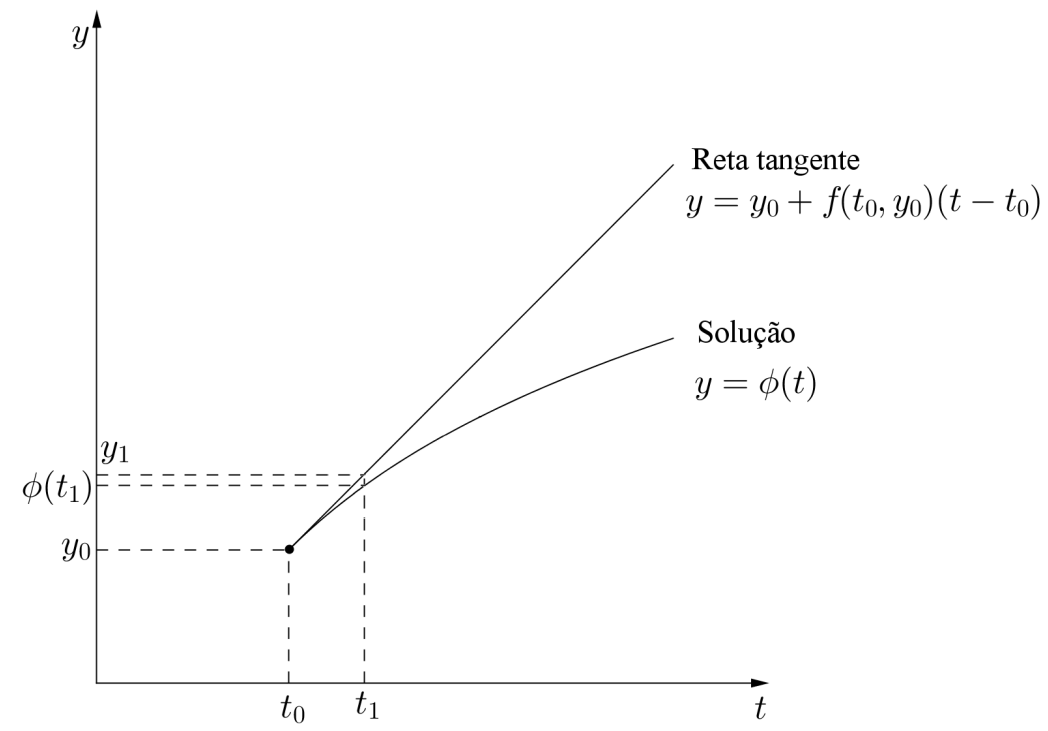

Fonte: Elaborada pelo autor.

Para continuar, tentaremos repetir o processo acima para encontrarmos uma aproximação num certo ponto $t_{2}$ próximo ao ponto $t_{1}$, mas temos o problema de não conhecermos o valor de $\phi\left(t_{1}\right)$. Entretanto, podemos usar o valor aproximado $y_{1}$, pois consideramos $y_{1}$ como uma boa aproximação de $\phi\left(t_{1}\right)$. Assim, consideramos a equação da reta que contém o ponto $\left(t_{1}, y_{1}\right) \mathrm{e}$ coeficiente angular $f\left(t_{1}, y_{1}\right)$, pois $y^{\prime}\left(t_{1}\right)=f\left(t_{1}, y\left(t_{1}\right)\right) \cong f\left(t_{1}, y_{1}\right)$, ou seja,

$$
y=y_{1}+f\left(t_{1}, y_{1}\right)\left(t-t_{1}\right) .
$$

Então, substituindo $t=t_{2}$, suficientemente próximo de $t_{1}$, nesta equação encontramos

$$
y_{2}=y_{1}+f\left(t_{1}, y_{1}\right)\left(t_{2}-t_{1}\right),
$$

que será uma aproximação de $\phi\left(t_{2}\right)$. 
Do mesmo modo, repetiremos o processo acima para encontrarmos uma aproximação num certo ponto $t_{3}$ próximo ao ponto $t_{2}$, usando o valor aproximado $y_{2}$, pois consideramos $y_{2}$ como uma boa aproximação de $\phi\left(t_{2}\right)$. Assim, consideramos a equação da reta que contém o ponto $\left(t_{2}, y_{2}\right)$ e coeficiente angular $f\left(t_{2}, y_{2}\right)$, ou seja,

$$
y=y_{2}+f\left(t_{2}, y_{2}\right)\left(t-t_{2}\right) .
$$

Então, substituindo $t=t_{3}$, suficientemente próximo de $t_{2}$, nesta equação encontramos

$$
y_{3}=y_{2}+f\left(t_{2}, y_{2}\right)\left(t_{3}-t_{2}\right),
$$

que será uma aproximação de $\phi\left(t_{3}\right)$.

Continuando o processo acima, note que o valor de $y$ calculado em cada etapa é usado para calcular o coeficiente angular da etapa seguinte e a expressão geral para a reta tangente passando por $\left(t_{n}, y_{n}\right)$ é

$$
y=y_{n}+f\left(t_{n}, y_{n}\right)\left(t-t_{n}\right) .
$$

Logo, o valor aproximado $y_{n+1}$ de $\phi\left(t_{n+1}\right)$ é dado por

$$
y_{n+1}=y_{n}+f\left(t_{n}, y_{n}\right)\left(t_{n+1}-t_{n}\right), \quad n=0,1,2, \ldots
$$

Agora, supondo que os pontos $t_{0}, t_{1}, t_{2}, \ldots$ estão igualmente espaçados e que $h$ é o número positivo constante equivalente ao espaçamento entre os pontos, temos que $t_{n+1}=t_{n}+h$. Neste caso, chamamos $h$ de tamanho do passo e a equação (5.2) pode ser escrita como

$$
y_{n+1}=y_{n}+h f\left(t_{n}, y_{n}\right), \quad n=0,1,2, \ldots
$$

O procedimento acima é conhecido como o método de Euler, e para usá-lo devemos calcular a equação (5.2) ou a equação (5.3) sistematicamente, escolhendo a equação conforme os passos sejam constantes ou não.

Exemplo 60. Considerando o P.V.I.

$$
\left\{\begin{array}{l}
y^{\prime}=3-2 t-\frac{1}{2} y, \\
y(0)=1,
\end{array}\right.
$$

usaremos o método de Euler, com passos de tamanho $h=0,2$, para encontrarmos uma aproximação da solução em $t=0,2 ; 0,4 ; 0,6 ; 0,8$ e 1 . Em seguida faremos uma comparação com os valores correspondentes da solução exata do P.V.I..

Neste caso, temos $f(t, y)=3-2 t-\frac{1}{2} y, t_{0}=0$ e $y_{0}=1$. Como $t_{0}=0$ e $h=0,2$, devemos utilizar 5 passos do método de Euler para encontrarmos uma aproximação da solução nos pontos 
dados. Para isso, notemos que

$$
\begin{aligned}
& y_{1}=y_{0}+h f\left(t_{0}, y_{0}\right)=1+0,2\left(\left(3-2(0)-\frac{1}{2}(1)\right)=1,5,\right. \\
& y_{2}=y_{1}+h f\left(t_{1}, y_{1}\right)=1,5+0,2\left(3-2(0,2)-\frac{1}{2}(1,5)\right)=1,87, \\
& y_{3}=y_{2}+h f\left(t_{2}, y_{2}\right)=1,87+0,2\left(3-2(0,4)-\frac{1}{2}(1,87)\right)=2,123, \\
& y_{4}=y_{3}+h f\left(t_{3}, y_{3}\right)=2,123+0,2\left(3-2(0,6)-\frac{1}{2}(2,123)\right)=2,2707, \\
& y_{5}=y_{4}+h f\left(t_{4}, y_{4}\right)=2,2707+0,2\left(3-2(0,8)-\frac{1}{2}(2,2707)\right)=2,32363 .
\end{aligned}
$$

Então, uma aproximação para a solução do P.V.I. em $t=0,2$ é $y_{1}=1,5$, em $t=0,4$ é $y_{2}=1,87$, em $t=0,6$ é $y_{3}=2,123$, em $t=0,8$ é $y_{4}=2,2707$, e em $t=1$ é $y_{5}=2,32363$. Mais ainda, a aproximação pela reta tangente perto de $t=0$ é

$$
y=1+f(0,1)(t-0)=1+2,5 t .
$$

Agora, note que a EDO do P.V.I. dado é linear e podemos resolvê-la usando o fator integrante $e^{\frac{t}{2}}$ e podemos verificar que a solução do P.V.I. é dada por

$$
y(t)=14-4 t-13 e^{-\frac{t}{2}} .
$$

Assim, temos que a solução exata do P.V.I. em $t=0,2$ é $y \cong 1,44$, em $t=0,4$ é $y \cong 1,7565$, em $t=0,6$ é $y \cong 1,9694$, em $t=0,8$ é $y \cong 2,0858$ e em $t=1$ é $y \cong 2,1151$.

A Figura 7 a seguir mostra a representação geométrica da solução e das aproximações dadas pelo método de Euler obtidas neste exemplo.

Figura 7 - Gráfico da solução e da aproximação pela reta tangente visto no Exemplo 60

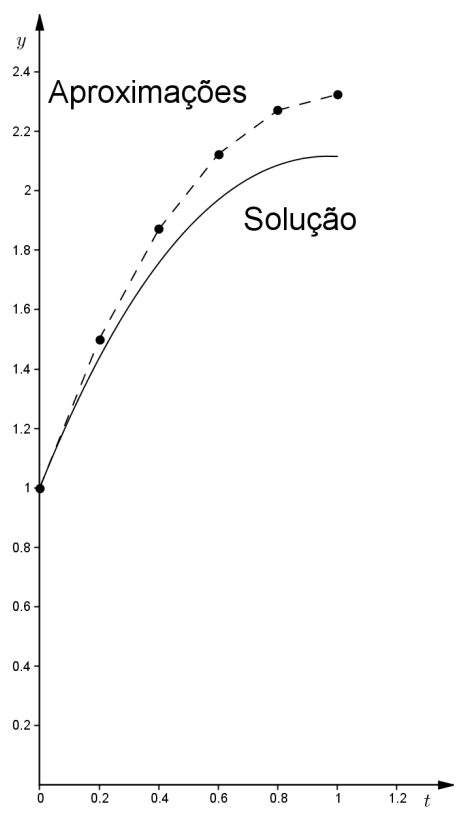

Fonte: Elaborada pelo autor. 
Exemplo 61. Consideremos o P.V.I.

$$
\left\{\begin{array}{l}
y^{\prime}=y, \\
y(0)=1 .
\end{array}\right.
$$

Usaremos o método de Euler para encontrarmos aproximações da solução do P.V.I dado, em $t=1$ usando passos de tamanhos $1,10^{-1}, 10^{-2}, 10^{-3}$ e $10^{-4}$.

Primeiramente, vejamos que

$$
\begin{aligned}
& y_{1}=y_{0}+h f\left(t_{0}, y_{0}\right)=y_{0}+h y_{0}=(1+h) y_{0}=(1+h), \\
& y_{2}=y_{1}+h f\left(t_{1}, y_{1}\right)=y_{1}+h y_{1}=(1+h) y_{1}=(1+h)(1+h)=(1+h)^{2}, \\
& y_{3}=y_{2}+h f\left(y_{2}, y_{2}\right)=y_{2}+h y_{2}=(1+h) y_{2}=(1+h)(1+h)^{2}=(1+h)^{3},
\end{aligned}
$$

e, por indução, é fácil ver que

$$
y_{n}=(1+h)^{n}, \quad n=1,2,3, \ldots
$$

Como $t_{0}=0$ e desejamos obter aproximações para $t=1$, devemos ter $n h=1$ e a equação (5.4) pode ser escrita como

$$
y_{n}=(1+h)^{\frac{1}{h}} .
$$

Logo, analisando cada passo temos

$$
\begin{aligned}
h=1 & \Rightarrow y=y_{1}=(1+1)^{\frac{1}{1}}=2^{1}=2 . \\
h=10^{-1} & \Rightarrow y=y_{10}=(1+0,1)^{\frac{1}{0,1}}=1,1^{10} \cong 2,59374 . \\
h=10^{-2} & \Rightarrow y=y_{100}=(1+0,01)^{\frac{1}{0,01}}=1,01^{100} \cong 2,70481 . \\
h=10^{-3} & \Rightarrow y=y_{1000}=(1+0,001)^{\frac{1}{0,001}}=1,001^{1000} \cong 2,71692 . \\
h=10^{-4} & \Rightarrow y=y_{10000}=(1+0,0001)^{\frac{1}{0,0001}}=1,0001^{10000} \cong 2,71815 .
\end{aligned}
$$

Por outro lado, sabemos que a solução exata desse P.V.I. é $y(t)=e^{t} \mathrm{e}$, portanto, para $t=1$ temos $y(1)=e$. Façamos uma análise comparando o tamanho do passo, a aproximação de Euler e o erro cometido na aproximação.

Tabela 1 - Análise do erro do método de Euler

\begin{tabular}{|c|c|c|}
\hline$h$ & Aproximação de Euler: $(1+h)^{\frac{1}{h}}$ & Erro: $e-(1+h)^{\frac{1}{h}}$ \\
\hline 1 & 2,00000 & 0,71828 \\
\hline $10^{-1}$ & 2,59374 & 0,12454 \\
\hline $10^{-2}$ & 2,70481 & 0,01347 \\
\hline $10^{-3}$ & 2,71692 & 0,00136 \\
\hline $10^{-4}$ & 2,71815 & 0,00014 \\
\hline
\end{tabular}

Observação 31. Na Tabela 1 dada no Exemplo 61 podemos perceber que o erro cometido na aproximação diminui a medida que tomamos o tamanho de passo cada vez menor. 
Em geral, o método de Euler não nos dará uma boa aproximação se os passos não forem muito pequenos. Logo, é natural nos perguntarmos como poderíamos melhorar este método. A próxima seção responde esta pergunta a partir de uma análise do próprio método.

\subsection{O Método de Euler Aperfeiçoado}

Considerando a equação do P.V.I. (5.1) e integrando os dois membros de $t_{n}$ a $t_{n+1}$ obtemos

$$
\begin{aligned}
\int_{t_{n}}^{t_{n+1}} y^{\prime}(t) d t & =\int_{t_{n}}^{t_{n+1}} f(t, y(t)) d t \\
y\left(t_{n+1}\right)-y\left(t_{n}\right) & =\int_{t_{n}}^{t_{n+1}} f(t, y(t)) d t \\
y\left(t_{n+1}\right) & =y\left(t_{n}\right)+\int_{t_{n}}^{t_{n+1}} f(t, y(t)) d t .
\end{aligned}
$$

Aqui $y(t)$ equivale à solução exata da equação e, assim, $y(t)=\phi(t)$. Então, temos a equação

$$
\phi\left(t_{n+1}\right)=\phi\left(t_{n}\right)+\int_{t_{n}}^{t_{n+1}} f(t, \phi(t)) d t .
$$

Como não podemos integrar $f(t, \phi(t))$ sem conhecer $\phi(t)$, teremos que aproximar essa integral. Para isso, podemos tentar a área sob o gráfico da função $f(t, \phi(t))$ pelo retângulo de base $t_{n+1}-t_{n}$ e altura $f\left(t_{n}, \phi\left(t_{n}\right)\right)$, como podemos ver na figura a seguir.

Figura 8 - Aproximação da integral por um retângulo

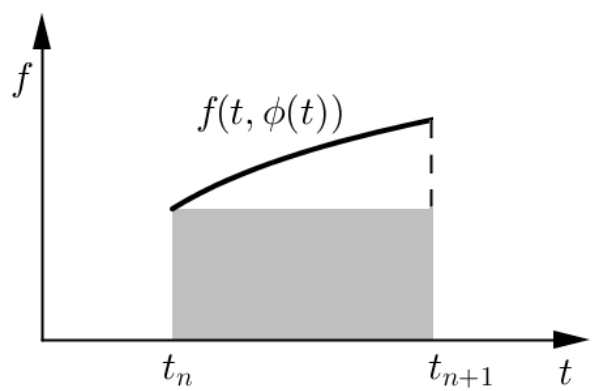

Fonte: Elaborada pelo autor.

Logo, a equação (5.5) pode ser reescrita como

$$
\phi\left(t_{n+1}\right)=\phi\left(t_{n}\right)+\left(t_{n+1}-t_{n}\right) f\left(t_{n}, \phi\left(t_{n}\right)\right) .
$$

Agora, supondo conhecida a aproximação $y_{n} \cong \phi\left(t_{n}\right)$ e tomando passos igualmente espaçados $h$, a equação acima fica

$$
y_{n+1}=y_{n}+h f\left(t_{n}, y_{n}\right), \quad n=0,1,2, \ldots,
$$

que é a fórmula de Euler obtida na seção anterior.

Entretanto, nosso objetivo é encontrar uma forma de melhorar o método de Euler. Logo, retomando o processo acima, vemos que isso pode ser feito se ao invés de aproximarmos a 
integral da função pela área do retângulo sob a função, aproximarmos pela área do trapezoide de bases $f\left(t_{n}, \phi\left(t_{n}\right)\right)$ e $f\left(t_{n+1}, \phi\left(t_{n+1}\right)\right)$ e altura $t_{n+1}-t_{n}$, como mostra a Figura 9 a seguir.

Figura 9 - Aproximação da integral por um trapezoide

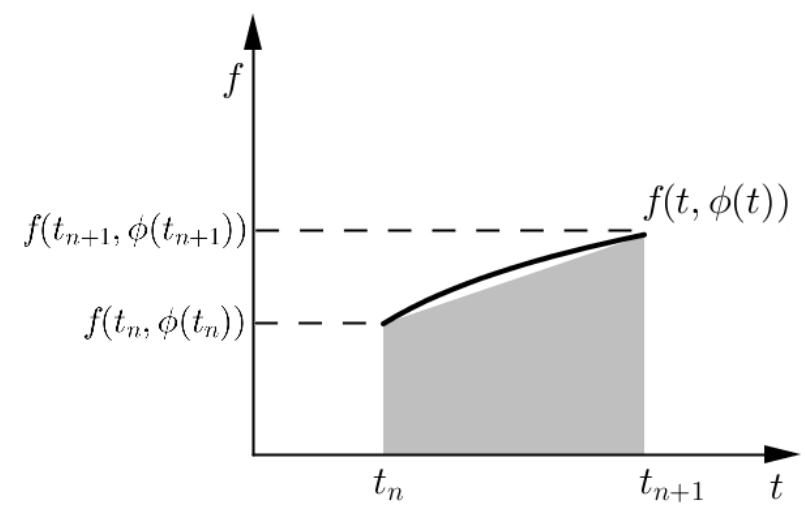

Fonte: Elaborada pelo autor.

Assim, a equação (5.5) pode ser reescrita na forma

$$
\phi\left(t_{n+1}\right)=\phi\left(t_{n}\right)+\frac{\left(t_{n+1}-t_{n}\right)\left[f\left(t_{n}, \phi\left(t_{n}\right)\right)+f\left(t_{n+1}, \phi\left(t_{n+1}\right)\right)\right]}{2} .
$$

Logo, supondo conhecida a aproximação $y_{n} \cong \phi\left(t_{n}\right)$, tomando passos igualmente espaçados e substituindo na equação acima obtemos

$$
y_{n+1}=y_{n}+\frac{h}{2}\left[f\left(t_{n}, y\left(t_{n}\right)\right)+f\left(t_{n+1}, y\left(t_{n+1}\right)\right)\right], \quad n=0,1,2, \ldots
$$

Note que a equação (5.7) é um método implícito para obter $y_{n+1}$, pois aparece como um argumento de $f$. Entretanto, podemos modificar esta equação de modo a torná-la um método explícito. Podemos fazer isso utilizando o método de Euler para aproximar o valor de $y_{n+1}$ no segundo membro da equação da seguinte forma

$$
y_{n+1}=y_{n}+\frac{h}{2}\left[f\left(t_{n}, y\left(t_{n}\right)\right)+f\left(t_{n}+h, y_{n}+h f\left(t_{n}, y_{n}\right)\right)\right], \quad n=0,1,2, \ldots
$$

Este método explícito é conhecido como método de Euler aperfeiçoado e é um método previsor-corretor, pois primeiro prevê o valor de $y_{n+1}$ pelo método de Euler e depois utiliza esse valor na equação (5.8) para uma aproximação melhor, ou seja, "mais correta".

Exemplo 62. Considerando o P.V.I.

$$
\left\{\begin{array}{l}
y^{\prime}=3-2 t-\frac{1}{2} y \\
y(0)=1
\end{array}\right.
$$

utilizaremos o método de Euler aperfeiçoado, com passos de tamanho $h=0,5$, para encontrarmos uma aproximação da solução em $t=1$. 
Como $h=0,5$ teremos 2 passos. Logo, sabendo que $f(t, y)=3-2 t-\frac{1}{2} y, t_{0}=0$ e $y_{0}=1$, temos

$$
\begin{aligned}
y_{1} & =y_{0}+\frac{h}{2}\left[f\left(t_{0}, y_{0}\right)+f\left(t_{0}+h, y_{0}+h f\left(t_{0}, y_{0}\right)\right)\right] \\
& =1+\frac{0,5}{2}[f(0,1)+f(0,5,1+0,5 f(0,1))] \\
& =1+0,25\left[\left(3-0 e-\frac{1}{2}\right)+\left(3-1-\frac{1}{2}\left(1+0,5\left(3-0-\frac{1}{2}\right)\right)\right)\right] \\
& =1,84375 \cong 1,844, \\
y_{2} & =y_{1}+\frac{h}{2}\left[f\left(t_{1}, y_{1}\right)+f\left(t_{1}+h, y_{1}+h f\left(t_{1}, y_{1}\right)\right)\right] \\
& =1,844+\frac{0,5}{2}[f(1,1,844)+f(1,5,1+0,5 f(1,1,844))] \\
& =1,844+0,25\left[\left(3-1-\frac{1,844}{2}\right)+\left(3-2-\frac{1}{2}\left((1,844)+0,5\left(3-1-\frac{1,844}{2}\right)\right)\right)\right] \\
& =2,065626 \cong 2,066 .
\end{aligned}
$$

Então, uma aproximação para a solução do P.V.I. em $t=1$ é $y=2,066$.

Note que o P.V.I. do Exemplo 62 é o mesmo do Exemplo 60, e que com apenas 2 passos o método de Euler aperfeiçoado fornece uma aproximação melhor que o método de Euler. Mais ainda, se utilizarmos o método de Euler aperfeiçoado com $h=0,2$, ou seja, 5 passos, obteremos como aproximação o valor 2,108, uma aproximação ainda melhor da solução exata em $t=1$.

Exemplo 63. Considere o P.V.I.

$$
\left\{\begin{array}{l}
y^{\prime}=y, \\
y(0)=1 .
\end{array}\right.
$$

Utilizaremos o método de Euler aperfeiçoado para encontrarmos aproximações em $t=1$ usando passos de tamanhos $1,10^{-1}, 10^{-2}$ e $10^{-3}$.

Primeiramente, note que

$$
\begin{aligned}
y_{1} & =y_{0}+\frac{h}{2}\left[f\left(t_{0}, y_{0}\right)+f\left(t_{0}+h, y_{0}+h f\left(t_{0}, y_{0}\right)\right)\right] \\
& =y_{0}+\frac{h}{2}\left[y_{0}+\left(y_{0}+h y_{0}\right)\right]=\left(1+h+\frac{h^{2}}{2}\right) y_{0}=\left(1+h+\frac{h^{2}}{2}\right), \\
y_{2} & =y_{1}+\frac{h}{2}\left[f\left(t_{1}, y_{1}\right)+f\left(t_{1}+h, y_{1}+h f\left(t_{1}, y_{1}\right)\right)\right] \\
& =y_{1}+\frac{h}{2}\left[y_{1}+\left(y_{1}+h y_{1}\right)\right]=\left(1+h+\frac{h^{2}}{2}\right) y_{1} \\
& =\left(1+h+\frac{h^{2}}{2}\right)\left(1+h+\frac{h^{2}}{2}\right)=\left(1+h+\frac{h^{2}}{2}\right)^{2},
\end{aligned}
$$




$$
\begin{aligned}
y_{3} & =y_{2}+\frac{h}{2}\left[f\left(t_{2}, y_{2}\right)+f\left(t_{2}+h, y_{2}+h f\left(t_{2}, y_{2}\right)\right)\right] \\
& =y_{2}+\frac{h}{2}\left[y_{2}+\left(y_{2}+h y_{2}\right)\right]=\left(1+h+\frac{h^{2}}{2}\right) y_{2} \\
& =\left(1+h+\frac{h^{2}}{2}\right)\left(1+h+\frac{h^{2}}{2}\right)^{2}=\left(1+h+\frac{h^{2}}{2}\right)^{3},
\end{aligned}
$$

e, por indução, é fácil verificar que

$$
y_{n}=\left(1+h+\frac{h^{2}}{2}\right)^{n}, \quad n=1,2,3, \ldots
$$

Como $t_{0}=0$ e desejamos obter aproximações da solução para $t=1$, devemos ter $n h=1$. Assim, a equação (5.9) pode ser escrita como

$$
y_{n}=\left(1+h+\frac{h^{2}}{2}\right)^{\frac{1}{h}} .
$$

Logo, analisando cada passo temos

$$
\begin{aligned}
& h=1 \Rightarrow y=y_{1}=\left(1+1+\frac{1^{2}}{2}\right)^{\frac{1}{1}}=2,5^{1}=2,5 \\
& h=10^{-1} \Rightarrow y=y_{10}=\left(1+0,1+\frac{0,1^{2}}{2}\right)^{\frac{1}{0,1}}=1,105^{10} \cong 2,71408 \\
& h=10^{-2} \Rightarrow y=y_{100}=\left(1+0,01+\frac{0,01^{2}}{2}\right)^{\frac{1}{0,01}}=1,01005^{100} \cong 2,71824 . \\
& h=10^{-3} \Rightarrow y=y_{1000}=\left(1+0,001+\frac{0,001^{2}}{2}\right)^{\frac{1}{0,001}}=1,0010005^{1000} \cong 2,71828 .
\end{aligned}
$$

Por outro lado, sabemos que a solução exata desse P.V.I. é $y(t)=e^{t}$ e, portanto, para $t=1$ temos $y(1)=e$. Façamos uma análise comparando o tamanho do passo, a aproximação aperfeiçoada de Euler e o erro cometido na aproximação.

Tabela 2 - Análise do erro do método de Euler aperfeiçoado

\begin{tabular}{|c|c|c|}
\hline$h$ & Aproximação: $\left(1+h+\frac{h^{2}}{2}\right)^{\frac{1}{h}}$ & Erro: $e-\left(1+h+\frac{h^{2}}{2}\right)^{\frac{1}{h}}$ \\
\hline 1 & 2,50000 & 0,21828 \\
\hline $10^{-1}$ & 2,71408 & 0,00420 \\
\hline $10^{-2}$ & 2,71824 & 0,00004 \\
\hline $10^{-3}$ & 2,71828 & 0,00000 \\
\hline
\end{tabular}

Observação 32. Comparando a Tabela 2 no Exemplo 63 com a Tabela 1 no Exemplo 61, vemos novamente que o método aperfeiçoado de Euler nos fornece uma aproximação muito mais precisa da solução exata. No entanto, a última linha da Tabela 2 deve ser analisada com cautela, pois tomamos aproximações com cinco casas decimais, tanto para o método quanto para o valor de $e$ e assim temos a sensação de não haver erro, o que não ocorre. 
Exemplo 64. Um químico possui 200 gramas de um elemento radioativo. Esse elemento tem variação anual de massa segundo a equação $m^{\prime}=-0,1733 m$. O químico deseja saber quantos gramas aproximadamente ele terá desse elemento daqui a 1 ano.

Usando o método de Euler teremos

$$
\begin{aligned}
& m_{1}=m_{0}+h\left(-0,1733 m_{0}\right)=m_{0}(1-0,1733 h)=200(1-0,1733 h), \\
& m_{2}=m_{1}+h\left(-0,1733 m_{1}\right)=m_{1}(1-0,1733 h)=200(1-0,1733 h)^{2}, \\
& m_{3}=m_{2}+h\left(-0,1733 m_{2}\right)=m_{2}(1-0,1733 h)=200(1-0,1733 h)^{3},
\end{aligned}
$$

e, por indução, é fácil ver que

$$
m_{n}=200(1-0,1733 h)^{n}
$$

Tomando, por exemplo, 5 passos temos

$$
m_{5}=200(1-0,1733(0,2))^{5} \cong 167,661 .
$$

Para 10 passos temos

$$
m_{10}=200(1-0,1733(0,1))^{10} \cong 167,922 .
$$

E com 20 passos temos

$$
m_{20}=200(1-0,1733(0,05))^{20} \cong 168,05 .
$$

Agora, usando o método de Euler aperfeiçoado teremos

$$
\begin{aligned}
m_{1} & =m_{0}+\frac{h}{2}\left[\left(-0,1733 y_{0}\right)+\left(-0,1733\left(y_{0}+h\left(-0,1733 y_{0}\right)\right)\right)\right. \\
& \left.=m_{0}\left(1-0,1733 h+0,015016445 h^{2}\right)\right] \\
& =200\left(1-0,1733 h+0,015016445 h^{2}\right), \\
m_{2} & =m_{1}+\frac{h}{2}\left[\left(-0,1733 y_{1}\right)+\left(-0,1733\left(y_{1}+h\left(-0,1733 y_{1}\right)\right)\right)\right. \\
& \left.=m_{1}\left(1-0,1733 h+0,015016445 h^{2}\right)\right] \\
& =200\left(1-0,1733 h+0,015016445 h^{2}\right)^{2}, \\
m_{3} & =m_{2}+\frac{h}{2}\left[\left(-0,1733 y_{2}\right)+\left(-0,1733\left(y_{2}+h\left(-0,1733 y_{2}\right)\right)\right)\right. \\
& \left.=m_{2}\left(1-0,1733 h+0,015016445 h^{2}\right)\right] \\
& =200\left(1-0,1733 h+0,015016445 h^{2}\right)^{3},
\end{aligned}
$$

e, por indução, é fácil ver que

$$
m_{n}=200\left(1-0,1733 h+0,015016445 h^{2}\right)^{n} .
$$

Logo, tomando 5 passos temos

$$
m_{5}=200\left(1-0,1733(0,2)+0,015016445(0,2)^{2}\right)^{5} \cong 168,183 .
$$


Agora, observando que a solução exata desse problema é dada por $m(t)=200 e^{-0,1733 t} \mathrm{e}$, então, $m(1)=200 e^{-0,1733} \cong 168,177$, podemos ver que o método de Euler aperfeiçoado fornece uma aproximação muito melhor com 5 passos que o método de Euler com 20 passos.

Exemplo 65. Suponha que determinada população tenha uma taxa de crescimento que varia com o tempo da seguinte forma

$$
\left\{\begin{array}{l}
\frac{d y}{d t}=\frac{(0,5+\operatorname{sen} t) y}{5} \\
y(0)=\alpha
\end{array}\right.
$$

Estimemos o instante no qual a população dobra de tamanho.

Como desejamos uma aproximação, tomamos passos de tamanho 1 , ou seja, $h=1$. Veremos que isso, além de não resultar em uma aproximação ruim, facilitará os cálculos. Faremos esta aproximação pelo método de Euler e também pelo método de Euler aperfeiçoado.

Pelo método de Euler temos

$$
y_{n+1}=y_{n}+h f\left(t_{n}, y_{n}\right)=y_{n}+y_{n} \frac{\left(0,5+\operatorname{sen} t_{n}\right)}{5} .
$$

Logo,

$$
\begin{aligned}
& y_{1}=\alpha+\alpha \frac{0,5+\operatorname{sen} 0}{5}=1,1 \alpha, \\
& y_{2}=1,1 \alpha+1,1 \alpha \frac{0,5+\operatorname{sen} 1}{5} \cong 1,395 \alpha, \\
& y_{3}=1,395 \alpha+1,395 \alpha \frac{0,5+\operatorname{sen} 2}{5} \cong 1,788 \alpha, \\
& y_{4}=1,788 \alpha+1,788 \alpha \frac{0,5+\operatorname{sen} 3}{5} \cong 2,017 \alpha .
\end{aligned}
$$

Pelo método de Euler aperfeiçoado temos

$$
\begin{aligned}
y_{n+1} & =y_{n}+\frac{h}{2}\left[f\left(t_{n}, y_{n}\right)+f\left(t_{n}+h, y_{n}+h f\left(t_{n}, y_{n}\right)\right)\right] \\
& =y_{n}+\frac{1}{2}\left\{\frac{\left(0,5+\operatorname{sen} t_{n}\right)}{5} y_{n}+\left[\frac{\left(0,5+\operatorname{sen} t_{n+1}\right)}{5}\right]\left[\frac{5,5+\operatorname{sen} t_{n}}{5}\right] y_{n}\right\} \\
& =y_{n}+y_{n} \frac{1}{2}\left\{\frac{\left(0,5+\operatorname{sen} t_{n}\right)}{5}+\left[\frac{\left(0,5+\operatorname{sen} t_{n+1}\right)}{5}\right]\left[\frac{5,5+\operatorname{sen} t_{n}}{5}\right]\right\} . \\
y_{1} & =\alpha+\alpha \frac{1}{2}\left\{\frac{(0,5+\operatorname{sen} 0)}{5}+\left[\frac{(0,5+\operatorname{sen} 1)}{5}\right]\left[\frac{5,5+\operatorname{sen} 0}{5}\right]\right\} \cong 1,198 \alpha, \\
y_{2} & =1,198 \alpha+1,198 \alpha \frac{1}{2}\left\{\frac{(0,5+\operatorname{sen} 1)}{5}+\left[\frac{(0,5+\operatorname{sen} 2)}{5}\right]\left[\frac{5,5+\operatorname{sen} 1}{5}\right]\right\} \cong 1,573 \alpha, \\
y_{3} & =1,573 \alpha+1,573 \alpha \frac{1}{2}\left\{\frac{(0,5+\operatorname{sen} 2)}{5}+\left[\frac{(0,5+\operatorname{sen} 3)}{5}\right]\left[\frac{5,5+\operatorname{sen} 2}{5}\right]\right\} \cong 1,924 \alpha .
\end{aligned}
$$

Concluímos, portanto, que a população dobra em aproximadamente 3 unidades de tempo. 
Agora, observamos que a solução exata desse P.V.I. é dada por

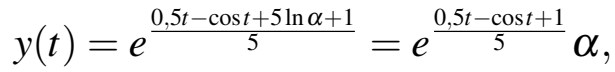

e daqui vemos que no terceiro período de tempo temos

$$
y(3)=e^{\frac{1,5-\cos 3+1}{5}} \alpha \cong 2,01 \alpha .
$$

Da solução exata vemos que $y(t)$ possui características de uma senoide, assim, há mais de um momento em que a população corresponde ao dobro da população inicial, sendo o primeiro deles em $t \cong 2,963$, com $y(2,963) \cong 1,9999$.

Podemos ver na Figura 10 a seguir uma comparação entre a solução exata, os valores obtidos pelo método de Euler, e os obtidos pelo método de Euler aperfeiçoado, para $\alpha=1$.

Figura 10 - Comparação das soluções

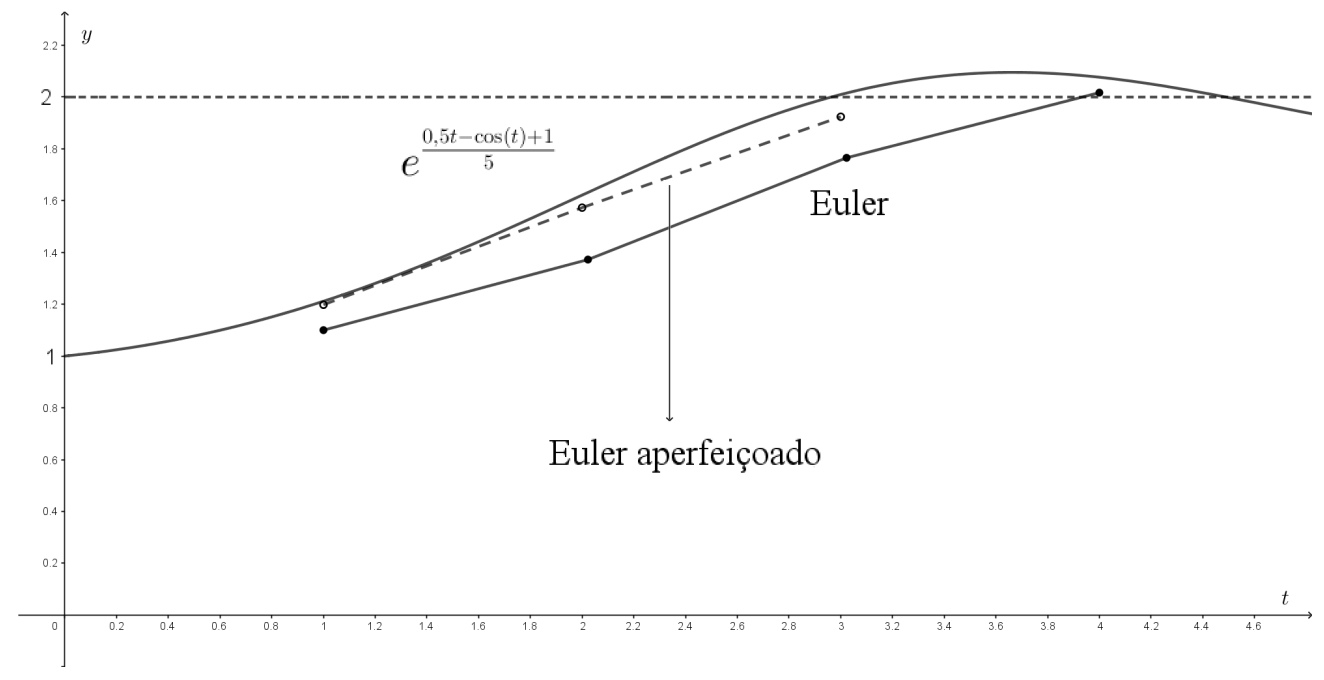

Fonte: Elaborada pelo autor.

\subsection{Uma Aplicação na Educação Básica}

Foram realizadas aulas com quatro alunos do $1^{\mathrm{o}}$ ano do ensino médio da Escola Estadual Nazle Jabur, situada na cidade de Passos em Minas Gerais.

A quantidade de alunos presentes deve-se ao fato de só esses possuírem interesse no assunto e disponibilidade para permanecer na escola após o horário de aula.

Nas aulas regulares os alunos haviam concluído recentemente o estudo das funções de primeiro grau, portanto, já possuíam conhecimento prévio a respeito de funções.

\subsubsection{A aplicação}

Primeiramente, foi apresentado aos alunos o conceito de derivada como uma taxa de variação e a relação desta com os modelos matemáticos, citando, como exemplo, que as popula- 
ções variam conforme passa o tempo e, relacionando com o que já haviam estudado em física, a distância percorrida variando de acordo com a velocidade.

Depois foi visto que os modelos que apresentam essas taxas de variações, ou seja, que possuem derivadas podem ser modelados por equações que recebem o nome de equações diferenciais. Então, foi explicado aos alunos a diferença entre as equações diferenciais ordinárias e as parciais e também a classificação das equações diferenciais pela ordem.

Em seguida, foi explicado aos alunos o método da aproximação de Euler e apresentado um exemplo modificado do Exemplo 61, em que a condição inicial era $y(0)=2$. Foram comparados os resultados encontrados para 5, 10 e 20 passos com o resultado exato.

Então houve uma abordagem sobre o método de Euler aperfeiçoado e foi apresentado um exemplo modificado do Exemplo 63 em que, novamente, a condição inicial era $y(0)=2$. Outra vez comparamos os resultados encontrados para 5, 10 e 20 passos com o resultado exato.

O Exemplo 65 foi feito passo-a-passo com os alunos. Dado isso os alunos foram divididos em duas duplas e apresentados aos seguintes problemas:

1. Um isótopo radioativo tem a variação de sua massa anual de acordo com a equação $m^{\prime}(t)=-0,2 m$. Quanto, um químico que possui inicialmente 200 gramas desse isótopo, terá daqui a 1 ano?

2. Uma colônia de bactérias cresce a uma razão proporcional ao número de bactérias presente, da seguinte forma $p^{\prime}=0,1 p$. Sabendo que, inicialmente, haviam 500 bactérias, quantas bactérias haverão depois de 6 horas?

Debatemos a respeito das características do resultado do problema 1 e uma das duplas resolveu usando o método de Euler para 5 e 10 passos, enquanto a outra resolveu usando o método de Euler aperfeiçoado também para 5 e 10 passos.

Do mesmo modo, procedemos, inicialmente, com o problema 2, debatendo a respeito das características do resultado. Então, os alunos tiveram que resolvê-lo usando o método de Euler. Nesse dia não foi possível uma das alunas participar da aula e, então, um aluno resolveu para 5 passos, outro para 10 e outro para 20.

Então, os alunos foram questionados a respeito de onde eles imaginavam o uso de equações diferencias ordinárias, e houve um debate sobre este assunto.

\subsubsection{Resultados}

Durante a explicação sobre o método de Euler, quando dito que ele gerava aproximações da resposta exata, os alunos questionaram se não era possível encontrar essa resposta exata e não aproximações, então foi-lhes explicado que para isso são necessários resultados de cálculo 
diferencial e integral e que esse não era um conteúdo estudado no ensino médio e que também há EDOs que não podemos encontrar a solução exata.

No momento de verificar as respostas referentes ao problema 1 foi possível verificar que a dupla responsável por utilizar o método de Euler aperfeiçoado apresentou mais dificuldade do que a dupla que utilizou o método de Euler, o que era esperado devido aquele possuir mais cálculos. Já no problema 2 não houve diferenças notáveis entre os resultados.

Quando questionados a cerca de onde eles imaginavam o uso de equações diferenciais ordinárias houve respostas como, por exemplo, a quantidade de mercadorias vendidas de acordo com o tempo e o dinheiro guardado na poupança mudar também de acordo com o tempo.

Foi comentado com os alunos que aquelas aulas faziam parte de um estudo a respeito da possibilidade de ensinar para alunos do ensino médio sobre equações diferenciais ordinárias. Os próprios alunos demonstraram interesse em saber como isso seria feito e comentaram que, com mais tempo, seria possível trabalhar melhor o assunto.

\subsubsection{Conclusão}

Acreditamos que os quatro alunos que fizeram parte desse estudo foram beneficiados uma vez que tiveram a oportunidade de ter contato com o estudo de um tópico de grande importância, principalmente para aqueles que desejam seguir caminho pela área das ciências exatas de algum modo.

Por fim, visto que os alunos mostraram bom desempenho e interesse, acreditamos que seria possível inserir o estudo das equações diferenciais no ensino médio, por meio de métodos numéricos, como, por exemplo, o método de Euler, devido os resultados apresentados, tendo como motivação as aplicações nas mais diversas áreas do conhecimento. 


\section{REFERÊNCIAS}

BOYCE, W. E.; DIPRIMA, R. C. Equações Diferenciais Elementares e Problemas de Valores de Contorno. 8. ed. Rio de Janeiro: LTC Editora, 2006. Citado na página 20.

BRAUN, M. Differential Equations and Their Applications. 4. ed. New York: Springer, 1992. Citado na página 20.

CHINCHIO, A. C. Introdução às equações diferenciais ordinárias e aplicações. Dissertação (Mestrado) - Universidade Estadual Paulista, Instituto de Geociências e Ciências Exatas, Rio Claro, 2012. Citado na página 20.

FARIA, S. R. de. A Catenária. Monografia (Especialização) - Universidade Federal de Minas Gerais, Belo Horizonte, 2011. Citado na página 20.

JUNIOR, H. C.; LADEIRA, L. A. da C. Equações Diferencias Ordinárias. São Carlos, 2011. Citado na página 20.

JUNIOR, L. P. L. Primeiro Relatório de Atividades - PICME Projeto: Equações Diferenciais e Aplicações. Ribeirão Preto, 2013. Citado na página 20.

NAGLE, R. K.; SAFF, E. B.; SNIDER, A. D. Equações Diferenciais. 8. ed. São Paulo: Pearson Education do Brasil, 2012. Citado na página 20.

TEIXEIRA, F. L. Modelos Descritos por Equações Diferenciais Ordinárias. Dissertação (Mestrado) - Universidade Estadual Paulista, Instituto de Geociências e Ciências Exatas, Rio Claro, 2012. Citado na página 20. 


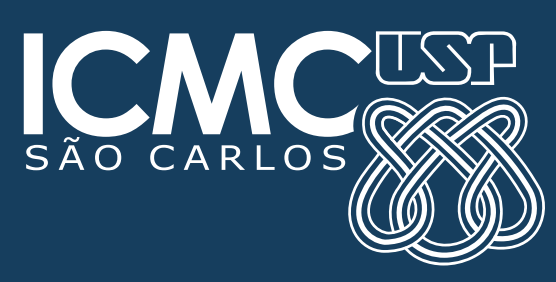

